\title{
Dust Eruptions
}

\author{
by \\ Harpreet Singh \\ Supervisor: Dr Mark McGuinness
}

A thesis submitted to Victoria University

in fulfillment of the requirements for the

degree of Master of Science

in the

Faculty of Science

School of Mathematics, Statistics and Operations Research

VICTORIA UNIVERSITY OF WELLINGTON

August 2014 


\section{Abstract}

We present a new model for the fragmentation of dust beds in laboratory shock tube experiments. The model successfully explains the formation of layers in the bed using mass and momentum conservation. Our model includes the effect of wall friction, inherent cohesion, and gravitational overburden. We find that the pressure changes caused by the expansion wave take time to penetrate into the bed, while simultaneously increasing in magnitude. By the time the pressure difference is large enough to overcome wall friction, the overburden and the intrinsic cohesion of the bed, it has penetrated $\sim 8-15$ bead diameters into the bed, thus causing a layer of dust to be lifted off. We have found the dependence of layer size upon bead diameter and found a good match to experiment. We have also predicted the dependence of layer size and fragmentation time on bead density. 


\section{Acknowledgements}

I'd like to thank first of all, my supervisor Dr Mark McGuinness.

I'd also like to thank my close friend Ashton Asbury, for continued support throughout this work. I am deeply grateful to my fellow students, particularly Susan Jowett, Jasmine Hall and Courtney Jones for encouragement during the writing process.

Finally, I owe my deepest gratitude to my family for putting up with me through all of my studies. 


\section{Contents}

Abstract

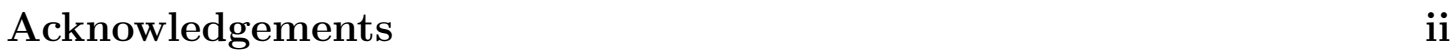

List of Figures $\quad$ v

List of Tables vi vi vis

1 Introduction $\quad 1$

1.1 Motivation . . . . . . . . . . . . . . . . . . 1

1.2 Background Theory . . . . . . . . . . . . . . . . . . 1

1.3 Experimental Review . . . . . . . . . . . . . . . . . . 3

1.4 Outline of Present Work . . . . . . . . . . . . . . . 6

2 Previous Work on Rocks $\quad 8$

3 Model Equations $\quad 11$

3.1 Dimensional Model Equations . . . . . . . . . . . . . . . . . . . 11

3.2 Rescaling . . . . . . . . . . . . . . . . . . . 13

3.3 Non-dimensional Model Equations . . . . . . . . . . . . . . . . 14

4 Diffusion Equations $\quad \mathbf{1 6}$

4.1 Linear Diffusion . . . . . . . . . . . . . . . . . . . . 16

4.1.0.1 Analytic Solutions . . . . . . . . . . . . 17

4.1.0.2 Numerical Solutions . . . . . . . . . . . . . 18

4.2 Non-linear Diffusion . . . . . . . . . . . . . . . . . 20

4.2 .1 Medium-velocity case . . . . . . . . . . . . . . . 20

4.2.1.1 Analytic Solutions . . . . . . . . . . . 20

4.2 .2 Numerical Solutions . . . . . . . . . . . . . . . . . 22

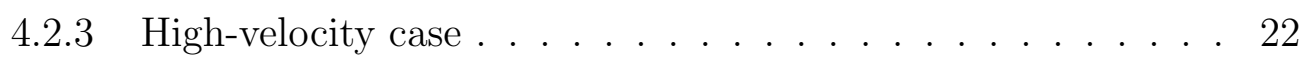

4.2.3.1 Analytic Solutions . . . . . . . . . . . . . 22

4.2.3.2 Numerical Solutions . . . . . . . . . . . . . . . . 24

4.2 .4 Non-diffusive behavior . . . . . . . . . . . . . . . 25

$5 \quad$ Wave-like Equations $\quad 27$ 
5.1 Analytic Solutions . . . . . . . . . . . . . . . . . . . . . . . . . 28

5.2 Numerical Solutions . . . . . . . . . . . . . . . . . . . 30

6 Tensile Strength and Hoop Stress 34

6.1 Tensile Strength . . . . . . . . . . . . . . . . . . . . 34

6.2 Hoop Stress . . . . . . . . . . . . . . . . 36

7 Dependence on Bead Diameter and Density 39

7.1 Dependence on particle diameter . . . . . . . . . . . . . . . 39

7.2 Dependence on bead density . . . . . . . . . . . . . . . 42

8 Conclusion $\quad 44$

$\begin{array}{ll}\text { Appendix } & 51\end{array}$ 


\section{List of Figures}

1.1 Set up of shock tube apparatus . . . . . . . . . . . . . . 4

1.2 An experimental run . . . . . . . . . . . . . . . . 5

1.3 Pressure profile . . . . . . . . . . . . . . . . 6

4.1 Numerical solution of linear diffusion problem . . . . . . . . . . . . 19

4.2 Numerical solution of linear diffusion problem-pressure difference . . 19

4.3 Numerical solution of linear diffusion problem-pressure difference with added $S_{o} \ldots \ldots \ldots \ldots \ldots \ldots$

4.4 Comparison of the analytic and numeric solutions to the linear diffusion problem .................... . . 21

4.5 Solution to medium velocity non-linear diffusion equation . . . . . . 23

4.6 Solution to medium velocity non-linear diffusion equation with pressure difference plotted . . . . . . . . . . . . . . . 23

4.7 Numerical solution of high-velocity diffusion . . . . . . . . . . . . 25

4.8 Numerical solution of high-velocity diffusion showing fragmentation point . . . . . . . . . . . . . . . 26

5.1 Numerical solution of the wave-like equation . . . . . . . . . . . . 31

5.2 Piecewise linear analytic solution of the wave-like equation . . . . . 31

5.3 Numerical solution of the wave-like problem showing the pressure difference . . . . . . . . . . . . . . . . 32

5.4 Numerical solution of wave-like equation for high velocity . . . . . . 32

5.5 Numerical solution with $\lambda \neq 0$. . . . . . . . . . . . . . 33

6.1 Linear diffusion with hoop stress . . . . . . . . . . . . . . . . 38

7.1 Linear diffusion with larger bead diameter . . . . . . . . . . . . . . 40

7.2 Linear diffusion with smaller bead diameter . . . . . . . . . . . . . . . . . . . . . . . 40

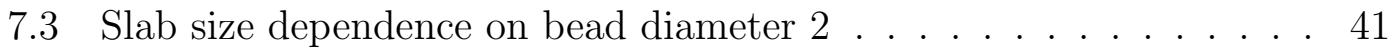

7.4 Slab size dependence on bead diameter 1 . . . . . . . . . . . . . 41

7.5 Comparison of model and experimental data . . . . . . . . . . . . . 42

7.6 Slab size dependence . . . . . . . . . . . . . . . . . . . . . 43

7.7 Dependence of fragmentation time on bead density . . . . . . . . . 43 


\section{List of Tables}

3.1 Physical constants . . . . . . . . . . . . . . . . . 13

3.2 Model Parameters . . . . . . . . . . . . . . . . . . . 15 


\section{Chapter 1}

\section{Introduction}

\subsection{Motivation}

This work is an explanation of the behavior of pressurized dust beds undergoing sudden and rapid decompression in a shock tube apparatus. The main objective of this research was to model and explain the fragmentation of fluidized beds in situations where the tensile strength of the bed is of the order of gravitational pressure. Rapid decompression refers to the depressurization brought about by shock tube rupturing which is discussed in Section 1.3. We have focused on the initial layer formation of the bed and not on the behavior of the bed after fragmentation.

\subsection{Background Theory}

Volcanic activity can occur along a spectrum of intensity, from low energy Hawaiian eruptions through highly energetic Volcanian to the very high energy Plinian and ultra-Plinian eruptions. The more energetic eruptions will eject more than $10^{6}$ $\mathrm{m}^{3}$ of ash and dust into the atmosphere and can result in disruption to air travel (such as with Eyjafjallajökull in 2010 or the 2011 Puyehue-Cordón Caulle eruption) or even world-wide cooling (Pinatubo in 1991 leading to 2-3 years of cooling) [1] and severe climatic disruption in the most extreme cases (The Tambora eruption of 1815, which lead to the 'year without a summer') [2]. The most well-studied of these eruptions are the eruptions of Mount St. Helens over 1980-1981 [3-6] and Eyjafjallajökull in 2010 [7][8]. 
These high-energy eruptions are frequently the result of a long build up of pressure and then a sudden depressurization due to the dislodging or collapse of the containing shield dome resulting in outflows of ash at speeds of hundreds of metres per second. The fragmentation mechanisms of the columns of dust and rock can be roughly divided into different groups depending on magma viscosity, which depends on silica content, temperature and the amount of dissolved water [9]. In low-viscosity magmas fragmentation mechanisms that have been proposed include bubble formation and foam instability. Bubble formation was first suggested as a mechanism by Verhoogen [10], who proposed that bubble density was the most important factor in ash formation. However, McBirney [11] found that the upward bubble velocity is slow enough that it is unlikely to disrupt the continuity of the solid. McBirney and Murase [9] modified the theory of Verhoogen to account for this and proposed that disruption occurs when the volume ratio of bubbles to magma becomes large. Sparks [12] further proposed that the bursting of bubbles caused explosive fragmentation. In high-viscosity magmas the bubble growth is heavily constrained by the viscous forces, resulting in over-pressurized vesicles. Bennett [13] proposes a mechanism that relies primarily on expansion waves and argues that Plinian eruptions can be modeled as one-dimensional shock tube experiment of the type described below (Section 1.3), however Sparks [12] argues that the bubbles internal to the magma would create their own expansion and compression waves and thus every bubble would act as its own diaphragm. However, the various bubbles may be small enough that their presence could be neglected in coarse models designed to model fragmentation alone. Valentine [14] argues that turbulence is more important to fragmentation than fluidization.

Fowler et al. [15] distinguished between the fragmentation caused by a rapidly accelerating two-phase flow and the fragmentation caused by rapid decompression. In the case of the rapidly accelerating two-phase flow, the most important mechanism relates again to bubble formation, which is confirmed by simulations by Papale [16]. This is most likely in low-viscosity magmas as seen in Hawaiian and Strombolian eruptions. Importantly, Papale finds an inverse relationship between viscosity at fragmentation, and porosity.

Rapid decompression is caused by the sudden removal of the object covering the top of the magma conduit, often a plug or dome. If a landslide dislodges the plug, or the pressure build-up within ejects it, rapid decompression will occur. Importantly, this is a far more transient and sudden process than with rapidly 
accelerating two-phase flow. This will result in an expansion wave of lower pressure (usually close to atmospheric pressure) traveling down the conduit fragmenting the solid magma in a layer by layer fashion. This can be explained as the pressure difference taking finite time to form across the rock, to an extent which it can overcome the tensile strength of the rock and thus cause it to fragment. A model for this is summarized in Chapter 2 .

This fragmentation phenomenon is related to layer formation and slug flow in fluidized bed reactors [17]. In chemical process reactors, fluidized beds allow fluid and particles to interact efficiently due to the high reaction surface area. The bed is usually packed on top of a porous screen through which the fluid flows. As the flow rate of the fluid increases the bed inflates and fluidization is the state when the flow rate is enough to support the particles against gravity. As the flow rate increases, bubbles form in the bed. Then at a threshold flow velocity, slugs form which are similar to the layers we are attempting to model below. The slugs are regions of particles separated by regions of mostly fluid. This behavior is considered undesirable as it results in less reactor efficiency. Above the threshold the bed becomes separated into slugs with a turbulent bubbly region above. The bubbles that separate the slugs rise in the bed in a similar manner to spherical cap bubbles in a low-viscosity fluid [18]. Beds in which the fluid is liquid instead of gas also display convection and circulation behavior, however this is outside the scope of this work [19-21]

Much attention in this area has also been paid to micro level interactions in systems of two and three particles. Inertia effects were found by Happel and Pfeffer [22] when they studied the interaction between two spheres following each other in a viscous fluid. They further found a definite attraction between the spheres and argue that the formation of these doublets would result in significantly increase the falling velocity of a bed of particles.

\subsection{Experimental Review}

In relation to fluidized beds the earliest experiments were done by Volpicelli et al. [23] and Zernow et al. [24]. Volpicelli et al. fluidized a single column of aluminum spheres using water and found large gaps just beyond the fluidization threshold. Fortes et al. [21] call these gaps 'void cracks'. Zernow et al. used a vertical shock 


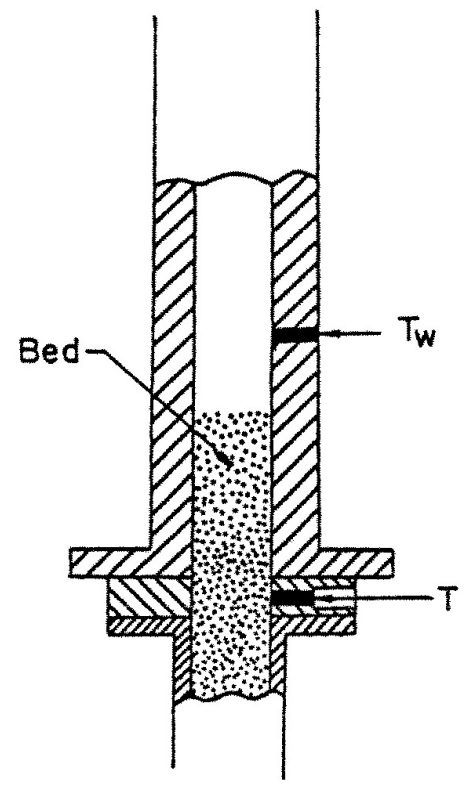

Figure 1.1: Set up of the shock tube apparatus in the Anilkumar experiements. Note that the pressure is measured at $T_{w}$.

tube similar to that used below, however the pressure release was much slower at 10 bar per second. This slow release has been modeled as a quasi-static process by Morrison [25] and by Nilson [26].

Experiments on bed of beads mobilised by a shock-tube apparatus have been described by Anilkumar et al. [27, 28] and are designed to inform our understanding of vulcanian eruptions. Cagnoli et al. [29] investigate the behavior of smaller beads under smaller pressures. The experimental setup is shown in Figure 1.1. Anilkumar's experiments used 90-2000 $\mu \mathrm{m}$ diameter, focusing on $125-1000 \mu \mathrm{m}$ and density of $2500 \mathrm{~kg} \mathrm{~m}^{-3}$. Cagnoli et al. used beads of average size 38 and $95 \mu \mathrm{m}$ of the same density.

The beads used in these experiments are Geldart group A and B type dusts [30]. Note that Geldart characterizes the powders based on steady-state fluidization experiments, while the shock tube experiments model a transient phenomenon. The smaller beads used in these experiments have the small mean size characteristic of group A powders. These tend to have far more inter-particle interaction and tends to expand quickly during fluidization. Group B powders tend to exhibit far less expansion and do not break from the slugging regime to the turbulent regime as the group A powders do. Most of Anilkumar's experiments work with Group $B$ powders while Cagnoli et al. uses beads that are close to the boundary between Groups A and B. 


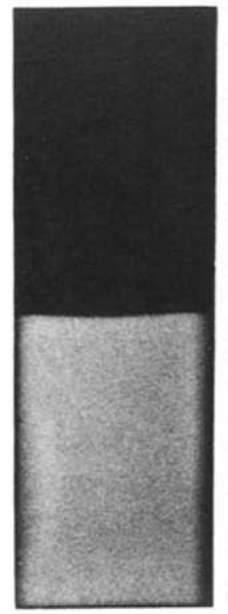

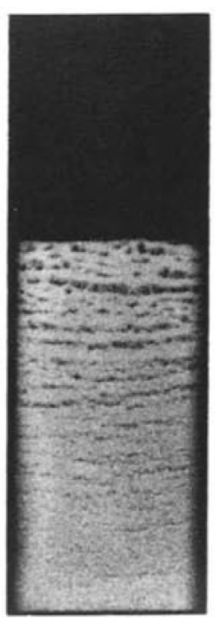

$2.8 \mathrm{~ms}$

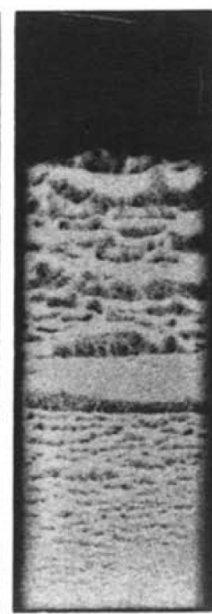

$4.0 \mathrm{~ms}$

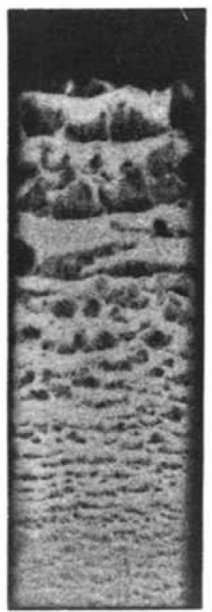

$5.2 \mathrm{~ms}$

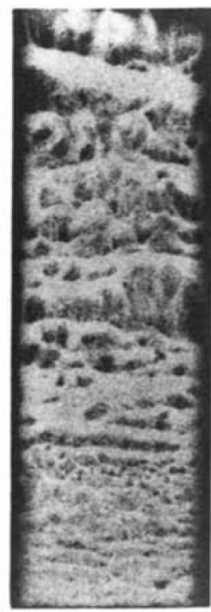

$6.5 \mathrm{~ms}$

Figure 1.2: An experimental run from [28] showing a rapid depressurization of the bed showing the formation of the slabs. The slabs form immediately with a thickness of approximately $15-20$ bead layers. Particle size is $125 \mu \mathrm{m}$ and the initial pressure is 3.1 bar.

In Anilkumar's experiments, packed beds of glass or steel spheres were pressurized to $\sim 2-4$ bar. The experiment was set up as in Figure 1.1. A diaphragm at the end was then ruptured, causing a sudden drop to atmospheric pressure there. After the diaphragm is ruptured an expansion wave travels down the tube to the sample leading to either to the lift off of slabs or if the experiment started at a low enough pressure, degassing of the entire sample [31], which is closer to the behavior seen in Zernow et al [24] without layer formation occurring.

Importantly, Anilkumar notes that the cracks that lead to slabs form immediately, without any initial expansion or change in bed porosity (Figure 1.2). This is very different behavior from that usually seen in the steady-state case, such as in reactors where inflation occurs before layer formation and is possibly to be a result of the transient nature of the rapid depressurization. Slab size is also observed to be independent of initial over-pressure (as long as the pressure is above the critical pressure for slab formation) and is proportional to the square root of sphere size. The measured pressure profile just above the bed gives an upper boundary condition for the model and is approximately exponential (Figure 1.3). We have assumed that the pressure at the top of the bed is the same as the pressure at $T_{w}$ and that because the bed does not expand before fragmentation, we see that the exponential decay is a good approximation. Further measurements show that the pressure at the bottom of the bed does not change over the first $12 \mathrm{~ms}$. Thus we can take the pressure at the base to be constant over the timescale 


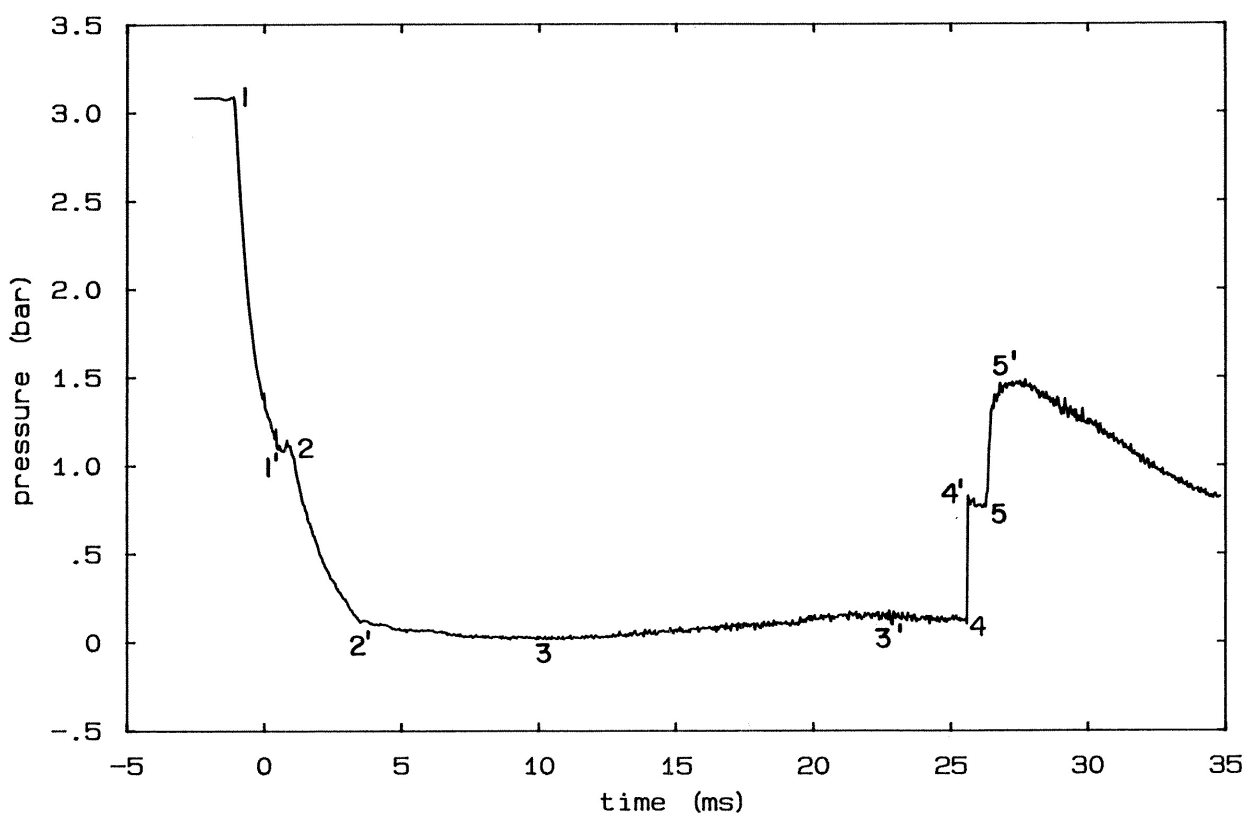

Figure 1.3: Pressure profile at $T_{w}$ in Anilkumar experiments. Over the first $3 \mathrm{~ms}$, it can be seen that the pressure follows an approximately exponential decay. The feature seen at point $4-4$ ' is the shock reflection from the top of the apparatus and the feature $5-5$ ' is the reflection from the surface of the bed.

that we are modeling. Anilkumar interprets the formation of these slabs as being due to dynamic processes, in which the first layer lifting creates a wake which lifts the next layer and so on. This entrainment continues until the layer of particles is too large to support itself leading to the separations that we see.

Cagnoli et al. observes with a smaller particle size, a lack of layer formation and irregular flow fronts. Thus our model should also not display layer formation at small bead diameters $(<100 \mu \mathrm{m})$. Further they see a more uniform bed inflation at small times than seen in Anilkumar.

\subsection{Outline of Present Work}

This work is organized as follows: Chapter 2 is a summary of previous modeling on rocks. This model is modified and applied to dust beds. Chapter 3 is a development from conservation equations of the model used in the rest of the work. This chapter includes derivation of relevant equations and nondimensionalization of those equations. Typical values of the physical constants and the model parameters are given here. Chapter 4 includes both analytic and numeric solutions for 
linear and non-linear diffusion equations that are obtained from reduction of the model obtained in chapter 3. Chapter 5 includes analytic and numeric solutions for wave-like equations that are obtained from reduction of the model obtained in chapter 3. Chapter 6 is a discussion of the sources of tensile strength in the fluidized beds and a derivation of wall friction in the form of 'hoop stress'. Chapter 7 is an analysis of the dependence of the layer size on particle diameter with comparison to experiment. Further we make predictions on the dependence of the layer size and fragmentation time on bead density. The Appendix includes a paper submitted to the Journal of Volcanology and Geothermal Research with a more sophisticated approach to wall friction. 


\section{Chapter 2}

\section{Previous Work on Rocks}

The equations that form the basis of the model used here were developed in Fowler et al [15] and McGuinness et al [32], though significant numerical simulation has also been done by Dartevelle \& Valentine [33]. The first two models consist primarily of conservation of mass and conservation of momentum for the pressurized gas and the solid. While we use the rock model as a base from which we develop the model for dusts, the rocks differ because they have much higher tensile strength. This leads to higher pressures $(\sim 100$ bar $)$ being needed to fragment the rocks. Furthermore, gravitational effects can be ignored as they are much smaller than the tensile strength. In the rock model (taken from Biot [34]) the solid small strain tensor is

$$
e_{i j}=\frac{1}{2}\left(\frac{\partial w_{i}}{\partial x_{j}}+\frac{\partial w_{j}}{\partial x_{i}}\right),
$$

where $w$ is the solid displacement. The dilatations of the solid and fluid are respectively defined by

$$
e=e_{k k}=\nabla \cdot w, \varepsilon=\nabla \cdot W
$$

where $W$ is the gas displacement. The equation for stresses then becomes

$$
(1-\phi) \sigma_{i j}^{S}=2 N e_{i j}+[A e+Q \varepsilon] \delta_{i j}
$$

and

$$
-\phi p=Q e+R \varepsilon,
$$


where $p$ is the gas pressure, $\phi$ is the porosity and $\sigma$ is the stress. Here $A$ and $N$ are Lamé constants for the solid and $Q$ and $R$ are related to the deformability of the pore space and fluid. Eliminating $\varepsilon$ allows equation 2.3 to be written in the form

$$
(1-\phi) \sigma_{i j}^{S}=2 N e_{i j}+[B e-\alpha p] \delta_{i j}
$$

The resulting conservation of momentum equation for the pore fluid is

$$
\rho_{f} \phi v_{t}=-\phi \nabla p-A-D
$$

and for the solid

$$
\rho_{s}(1-\phi) u_{t t}=(1-\phi) \nabla \cdot \sigma^{S}+A+D
$$

where $\rho_{f}$ and $\rho_{s}$ are the fluid and solid densities, $v$ is the gas velocity, $u$ is the solid displacement from equilibrium, $A$ represents the added mass effect, which is due to the movement of the beads in a fluid, which also results in the fluid being displaced backwards, and is taken by Biot to be

$$
A=\rho_{a} \frac{\partial}{\partial t}\left(v-u_{t}\right)
$$

and $D$ is the interfacial drag and taken to be

$$
D=b\left(v-u_{t}\right)
$$

where $b$ is the interfacial drag coefficient. Darcy's law is obtained by ignoring the usually small acceleration terms and setting

$$
b=\frac{\eta_{f} \phi^{2}}{k}
$$

(from [34]) with $\eta_{f}$ as the gas viscosity and $k$ is the gas permeability. To include turbulent (Ergun) flow, the drag can be found using the Forchheimer equation

$$
-\nabla p=\frac{\eta_{f} V}{k}+\frac{\rho_{f} c_{F}|V| V}{\sqrt{k}},
$$

where $V=v-u_{t}[35]$. Conservation of mass for the gas gives

$$
\left(\rho_{f} \phi\right)_{t}+\nabla \cdot\left(\rho_{f} \phi v\right)=0
$$


where $\phi$ is the porosity. Similarly conservation for the solid gives

$$
\left((1-\phi) \rho_{s}\right)_{t}+\nabla \cdot\left((1-\phi) \rho_{s} u_{t}\right)=0
$$

The state equation for an ideal gas gives

$$
\frac{\rho_{f}}{\rho_{o}}=\left(\frac{p}{p_{o}}\right)^{\frac{1}{\gamma}}
$$

where $\gamma$ is the adiabatic constant. After non-dimensionalisation and the small terms are set to zero, the pressure satisfies the nonlinear diffusion equation

$$
\frac{\partial p^{\frac{1}{\gamma}}}{\partial t}=\frac{\partial}{\partial z}\left(\left|\frac{p^{\frac{1}{\gamma}}}{p_{z}}\right|^{\frac{1}{2}} \frac{\partial p}{\partial z}\right),
$$

with the adiabatic law becoming

$$
\rho=p^{\frac{1}{\gamma}}
$$

which allowed equation 2.15 to be converted into

$$
\frac{\partial \rho}{\partial t}=\frac{\partial}{\partial z}\left(\left|\frac{\gamma \rho^{\gamma}}{\rho_{z}}\right|^{\frac{1}{2}} \frac{\partial \rho}{\partial z}\right)
$$

which is simpler for numerical purposes. 


\section{Chapter 3}

\section{Model Equations}

\subsection{Dimensional Model Equations}

The initial model will consist of mass and momentum conservation equations for the gas and the packed bed. To this we will add an equation of state for the gas. This model will closely follow the modeling approach of the previous chapter. The momentum conservation equation for the gas is

$$
\rho \phi v_{t}=-\phi p_{z}-A-D
$$

where $\rho$ is the gas density, $\phi$ is the porosity of the dust bed, $v$ is the gas velocity and $p$ is the absolute gas pressure. $A$ is the added mass effect which is caused by the gas being displaced as the beads move and takes the form

$$
A=\theta C_{v m} \rho\left(v_{t}-u_{t}\right)
$$

where $u$ is the bead velocity and $C_{v m}$ is an order one constant relating the added mass density to the porosity and gas density. It is important to note that we have switched from bead displacement $w$ (as in the previous section) to bead velocity $u$. Further as we will be mostly dealing with the gas behaviour we have replaced $\rho_{f}$ with $\rho . D$ is the inter-facial drag as the gas moves past the beads and has dimensions of pressure gradient and $\theta=(1-\phi)$. It is found by extending Darcy's law to account for turbulent flow (Forcheimer's or Ergun's equation) and takes the form

$$
D=\frac{\eta \phi^{2}}{k}(v-u)+\frac{\rho C_{F} \phi^{3}}{\sqrt{k}}(v-u)|v-u|
$$


where $\eta$ is the dynamics viscosity of the gas (about $18 \times 10^{-6}$ Pa.s), $\mathrm{k}$ is the bed permeability (about $10 \times 10^{-10} \mathrm{~m}^{2}$ ) and $C_{F}$ is the dimensionless Ergun coefficient (about 0.5 ), and $\mathrm{k}$ is the permeability.

For the beads, momentum conservation gives

$$
\rho_{s} \theta u_{t}=-\theta p_{z}+A+D-\rho_{s} \theta g
$$

where $\rho_{s}$ is the bead density, $u$ is the bead velocity and $g$ is the acceleration due to gravity. We have used bead velocity instead of bead displacement in contrast to Fowler et al because we are not modelling the stress-strain relationship on the bed. Conservation of gas mass is given by

$$
(\rho \phi)_{t}+(\rho \phi v)_{z}=0
$$

and if we assume adiabatic expansion of the gas, we have

$$
\rho=\rho_{o}\left(\frac{p}{p_{o}}\right)^{\frac{1}{\gamma}}
$$

where $\gamma$ is the adiabatic index (1.4 for nitrogen), and $\rho_{o}$ and $p_{o}$ are the initial values of gas density and pressure. We can assume this because the expansion of the gas is over a very short timescale. Mass conservation for the beads gives

$$
\left(\rho_{s} \theta\right)_{t}+\left(\rho_{s} \theta u\right)_{z}=0
$$

Finally, we have the initial conditions of $v=0, p=p_{o}, u=0$ and $\phi=\phi_{o}=0.4$ at $t=0$ and the boundary conditions of $p_{z}=0$ at $z=0$ and

$$
p=p_{c}(t)=\left(p_{o}-p_{a}\right) \exp \left(\frac{-t}{t_{c}}\right)+p_{a}
$$

where $p_{a}$ is atmospheric pressure and $t_{c}$ is the characteristic time scale of the decay (approximately $3 \mathrm{~ms}$ ). As we are concerned only with initiation of fragmentation, we take $\phi$ to be equal to $\phi_{o} \approx 0.4$. [36] We suppose that the bed will rupture when the pressure gradient exceeds the gravitational overburden plus a small effective tensile strength. This condition can be written as

$$
p_{b}(z)-p_{c}(t) \geq S_{o}+\theta \rho_{s} g \begin{cases}(l-z), & 0<z<l-D_{p} \\ D_{p}, & l-D_{p} \leq z<l\end{cases}
$$




\begin{tabular}{|c|l|l|l|l|}
\hline Symbol & Meaning & Range & Typical Value & Unit \\
\hline$c_{F}$ & Ergun coefficient & & 0.5 & Dimensionless \\
$C_{V M}$ & added mass constant & & 1 & Dimensionless \\
$D_{p}$ & bead diameter & $30-1000$ & 500 & $\mu \mathrm{m}$ \\
$k$ & permeability & $(4-40) \times 10^{-11}$ & $16 \times 10^{-11}$ & $\mathrm{~m}^{2}$ \\
$l$ & bed depth & $0.02-0.64$ & 0.04 & $\mathrm{~m}$ \\
$p_{a}$ & initial gas pressure & $1-3$ & 2 & $\mathrm{bar}$ \\
$t_{c}$ & chamber relaxation time & $1-3 \times 10^{-3}$ & $2 \times 10^{-3}$ & $\mathrm{~s}$ \\
$\gamma$ & specific heat ratio & & 1.4 & $\mathrm{Dimensionless}$ \\
$\rho_{o}$ & initial gas density & $1-2.3$ & 2.3 & $\mathrm{~kg} \cdot \mathrm{m}^{-3}$ \\
$\rho_{s}$ & solid density (glass) & & $2.5 \times 10^{3}$ & $\mathrm{~kg} \cdot \mathrm{m}^{-3}$ \\
$\nu$ & gas viscosity & $1.8 \times 10^{-5}$ & $\mathrm{~Pa} \cdot \mathrm{s}$ \\
$\phi_{o}$ & initial porosity & & 0.4 & $\mathrm{Dimensionless}$ \\
\hline
\end{tabular}

TABLE 3.1: Physical constants used in the model with ranges, typical values and units.

$S_{o}$ is the tensile strength of the bulk powder and could take multiple forms. For now we shall take it as $\rho_{s} g \frac{D_{p}}{2}$ from Weir [37] but its form is further discussed below (Chapter 6).

We take $k$ as subject to the Carman-Kozeny Relationship:

$$
k=\frac{D_{p}^{2} \phi^{3}}{72 \tau \theta^{2}}
$$

where $D_{p}$ is the bead diameter and $\tau$ is the tortousity or roughness of the spheres, calculated using the arc-chord ratio. This relationship will change at high porosities, but these are beyond the model we are using here. They are further discussed in Kobayashi et al [38].

\subsection{Rescaling}

The equations are rescaled using the following transformations with the nondimensional variables with a tilde. 


$$
\begin{aligned}
\rho & =\rho_{o}(1-\lambda \tilde{\rho}) & k & =k_{o} \tilde{k} \\
p & =p_{o}(1-\gamma \lambda \tilde{p}) & z & =l \tilde{z} \\
p_{a} & =p_{o}\left(1-\gamma \lambda \tilde{p}_{a}\right) & u & =u_{o} \tilde{u} \\
\lambda & =\frac{\rho_{s} g l}{\gamma p_{o}} & u_{o} & =\frac{D_{p}}{t_{o}} \\
t & =t_{o} \tilde{t} & D_{p} & =l \tilde{D}_{p} \\
t_{o} & =\frac{\lambda l}{v_{o}} & A & =A_{o} \tilde{A} \\
v & =v_{o} \tilde{v} & D & =D_{o} \tilde{D} \\
\phi & =\phi_{o} \tilde{\phi} & &
\end{aligned}
$$

The time scale used is the time over which the pressure follows an exponential decay. There are two important length scales: the depth of the bed and the size of the beads. The scaling chosen for $z$ is the depth of the bed as the pressure change is occurring over many bead diameters. The pressure and density scalings are chosen such that the adiabatic law reduces to $\rho \approx p$ when $\lambda$ is small. This allows considerable simplification compared to the rock modeling as the scaling used in [15] gives equation 2.16. $\lambda$ is of the order of the gas overburden which is the pressure gradient of the gas due to gravity. By convention the tilde notation will be dropped now, with all variables being non-dimensional unless otherwise stated.

\subsection{Non-dimensional Model Equations}

The model equations become (with tildes omitted)

$$
\begin{gathered}
\nu \phi(1-\lambda \rho) v_{t}=\phi p_{z}-\nu A-D \\
\frac{\partial}{\partial t}[(1-\lambda \rho) \phi]=\frac{\partial}{\partial z}[\lambda(1-\lambda \rho) \phi v] \\
\beta_{1} u_{t}=\theta p_{z}+\nu A+D-\beta_{2} \theta \\
\phi_{t}=\beta_{3} \frac{\partial}{\partial z}[\theta u] \\
A=\theta C_{V M}(1-\lambda \rho)\left(v_{t}-\epsilon u_{t}\right) \\
D=\frac{\phi(v-\epsilon u)}{k}+\delta \phi^{3}(1-\lambda \rho)\left(\frac{(v-\epsilon u)|v-u|}{\sqrt{k}}\right)
\end{gathered}
$$




\begin{tabular}{|l|c|r|}
\hline Parameter & Formula & Typical Value \\
\hline$a$ & $\frac{t_{o}}{t_{c}}$ & 0.08 \\
$\lambda$ & $\frac{\left(\theta_{o}\right) \rho_{s} g l}{\gamma p_{o}}$ & 0.002 \\
$\delta$ & $\frac{\nu \lambda l C_{F}}{\sqrt{k_{o}}}$ & 0.6 \\
$t_{o}$ & $2.5 \mathrm{~ms}$ \\
$v_{o}$ & $\frac{\lambda l}{v_{o}}$ & $0.3 \mathrm{~ms}^{-1}$ \\
$\beta_{1}$ & $\frac{k_{o} p_{o} \gamma \lambda}{\nu_{o} l}$ & 1350 \\
$\beta_{2}$ & $\frac{D_{p}}{t_{o}^{2}\left(\theta_{o}\right) g}$ & 0.01 \\
$\beta_{3}$ & $\frac{u_{o} t_{o}}{l}$ & 1 \\
\hline
\end{tabular}

TABLE 3.2: Parameters of the nondimensionalized model

The rupture criterion becomes

$$
p_{c}-p \geq S_{o}+\theta \begin{cases}(1-z), & 0<z<1-D_{p} \\ D_{p}, & l-D_{p} \leq z<1\end{cases}
$$

with the boundary and initial conditions for pressure becoming

$$
p_{z}(0, t)=0, p(z, 0)=0,
$$

and

$$
p(1, t)=p_{a}\left(1-e^{-a t}\right),
$$

while the boundary and initial conditions for the other variables become

$$
v(0, t)=0, v(z, 0)=0, u(z, 0)=0 \text { and } \phi(z, 0)=\phi_{o}=0.4
$$

Note that we have applied the conditions on $p_{z}(0, t)$ and $v(0, t)$ because there is no flow through the bottom of the container and the pressure there does not change over the timescale that we are modelling. 


\section{Chapter 4}

\section{Diffusion Equations}

We can reduce these equations to various diffusion cases, depending on the assumptions made. If we take $\lambda, \nu, \delta, \epsilon, \beta_{1}, \beta_{2}$ and $\beta_{3}$ to be zero we reduce to the linear diffusion case. If we examine the situation where the bed has not expanded we then have $\delta$ is small compared to the linear term in the drag and we can treat the problem as a force balance between the drag and the pressure gradient to obtain a linear diffusion equation.

\subsection{Linear Diffusion}

For the stationary bed, initially the drag reduces to

$$
D=\frac{\phi v}{\phi_{o} k}
$$

The gas mass equation becomes

$$
p_{t}=v_{z}
$$

allowing us to obtain

$$
p_{z}=\frac{\phi^{2} v}{\phi_{o} k}
$$

The physical interpretation of this, is that the pressure gradient balances with the drag. We can substitute into 4.2 to obtain

$$
p_{t}=\phi_{o}\left(\frac{k p_{z}}{\phi}\right)_{z}
$$


Before fracturing we can take $\phi=\phi_{o}$ and $k$ is the scaled permeability, which we can take to be constant and equal to 1 for now. Thus the equation reduces to the linear diffusion equation

$$
p_{t}=p_{z z}
$$

We have initial conditions $p=0$ at $t=0$ and boundary conditions $p_{z}=0$ at $z=0$ and $p=p_{c}=p_{a}(1-\exp (-a t))$ at $z=1$. This is separable into equations for space and time, and can be matched to the boundary conditions for small t using $p_{c}=p_{a}(1-\exp (-a t)) \approx a t$.

\subsubsection{Analytic Solutions}

If we take a Laplace transform in time

$$
P(z, s)=\int_{0}^{\infty} p(z, t) e^{-s t} d s
$$

we find that the differential equation transforms to

$$
P_{z z}=s P
$$

with boundary conditions $P_{z}=0$ at $z=0$ and $P=\frac{a p_{a}}{s(s+a)}$ at $z=1$. The solution in transform space is

$$
P(z, s)=\frac{a p_{a}}{s(s+a)} \frac{\cosh (\sqrt{s} z)}{\cosh (\sqrt{s})}
$$

from Crank [39] we can invert this which gives an infinite sum of erfc functions which converge rapidly for all except large values of $t$. For small times we proceed by expanding the hyperbolic functions into exponentials

$$
P=\frac{a p_{a}}{s(s+a)} \frac{e^{\sqrt{s} z}+e^{-\sqrt{s} z}}{e^{\sqrt{s}}\left(1+e^{-2 \sqrt{s}}\right)}
$$

then converting them into a sum using the Binomial Theorem

$$
P=\frac{a p_{a}}{s(s+a)}\left(e^{-\sqrt{s}(1-z)}+e^{-\sqrt{s}(1+z)}\right) \sum_{n=0}^{\infty}(-1)^{n} e^{-2 n \sqrt{s}}
$$

Then

$$
P=\frac{a p_{a}}{s(s+a)}\left(\sum_{n=0}^{\infty}(-1)^{n} e^{-\sqrt{s}(2 n+1-z)}+\sum_{n=0}^{\infty}(-1)^{n} e^{-\sqrt{s}(2 n+1+z)}\right)
$$


which for large $s$ (small $t$ ) converges to

$$
P \sim a p_{a}\left(\frac{e^{-\sqrt{s}(1-z)}}{s^{2}}\right)
$$

which has the inverse transform [40]

$$
p(z, t)=4 a p_{a} t \mathrm{i}^{2} \operatorname{erfc}\left(\frac{1-z}{2 \sqrt{t}}\right)
$$

where:

$$
\mathrm{i}^{2} \operatorname{erfc}(x)=\frac{1}{\pi} \int_{x}^{\infty}(t-x)^{2} e^{-t^{2}} d t
$$

is the integrated complementary error function This can be converted to the form

$$
p(z, t)=a p_{a}\left[\left(t+\frac{(1-z)^{2}}{2}\right) \operatorname{erfc}\left(\frac{1-z}{2 \sqrt{t}}\right)-(1-z) \sqrt{\frac{t}{\pi}} e^{\frac{(1-z)^{2}}{4 t}}\right]
$$

where $\operatorname{erfc}(x)$ is the complementary error function

$$
\operatorname{erfc}(x)=1-\operatorname{erf}(x)=\int_{x}^{\infty} e^{-t^{2}} d t
$$

Further, we can find the gas velocity by using the relation

$$
v=\int p_{t} d z
$$

This will be compared to the numerical solution below (Figure 4.4).

\subsubsection{Numerical Solutions}

Solving the system numerically shows that the expansion wave propagates inward from the surface (Figure 4.1). When $S_{o}$ is zero in 3.18, the model predicts dust fragmentation immediately from the first bead as shown. This obviously does not match the experimental observation. When $S_{o}$ is non-zero fragmentation occurs further in, at $z \approx 0.96$ which gives slabs of $4-8$ beads (Figure 4.3 ). This is closer to the experimental value than before, but the layers are still $30-50 \%$ too small. 


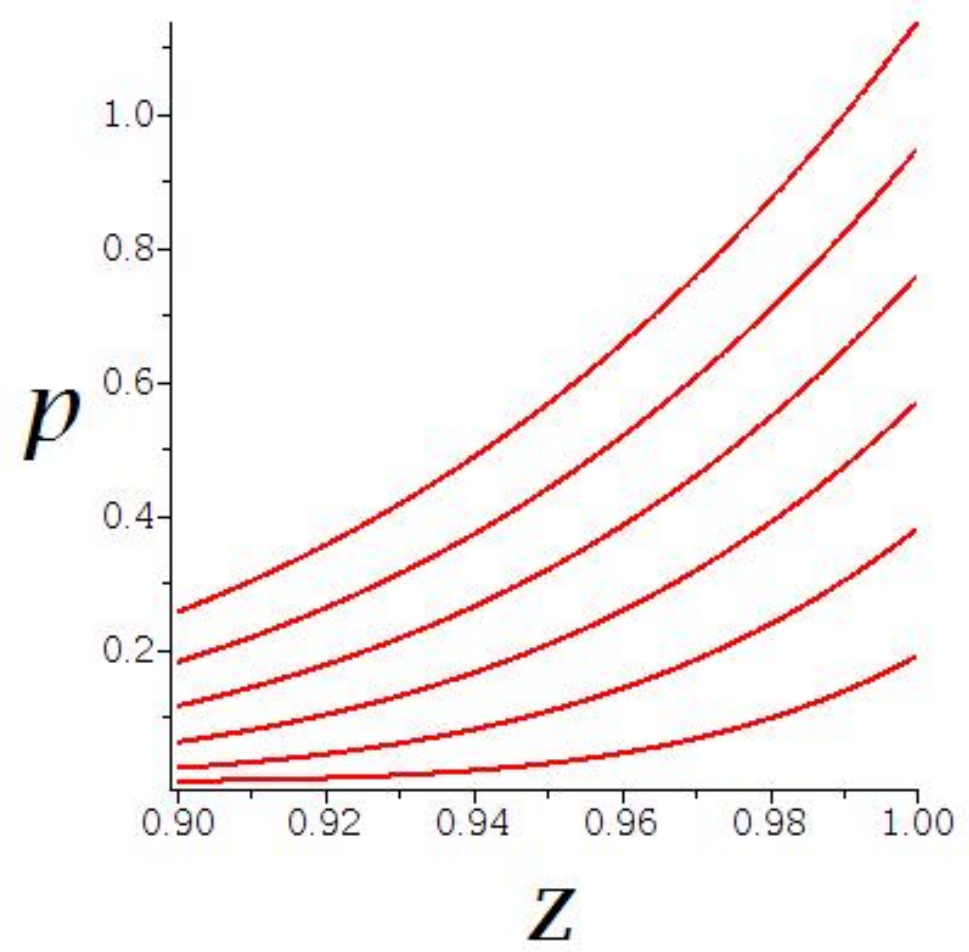

Figure 4.1: The numerical solution to the linear diffusion problem plotted in equal intervals from $t=0.00125$ to $t=0.0075$. The point of maximum pressure gradient is never at the surface but in the interior of the bed.

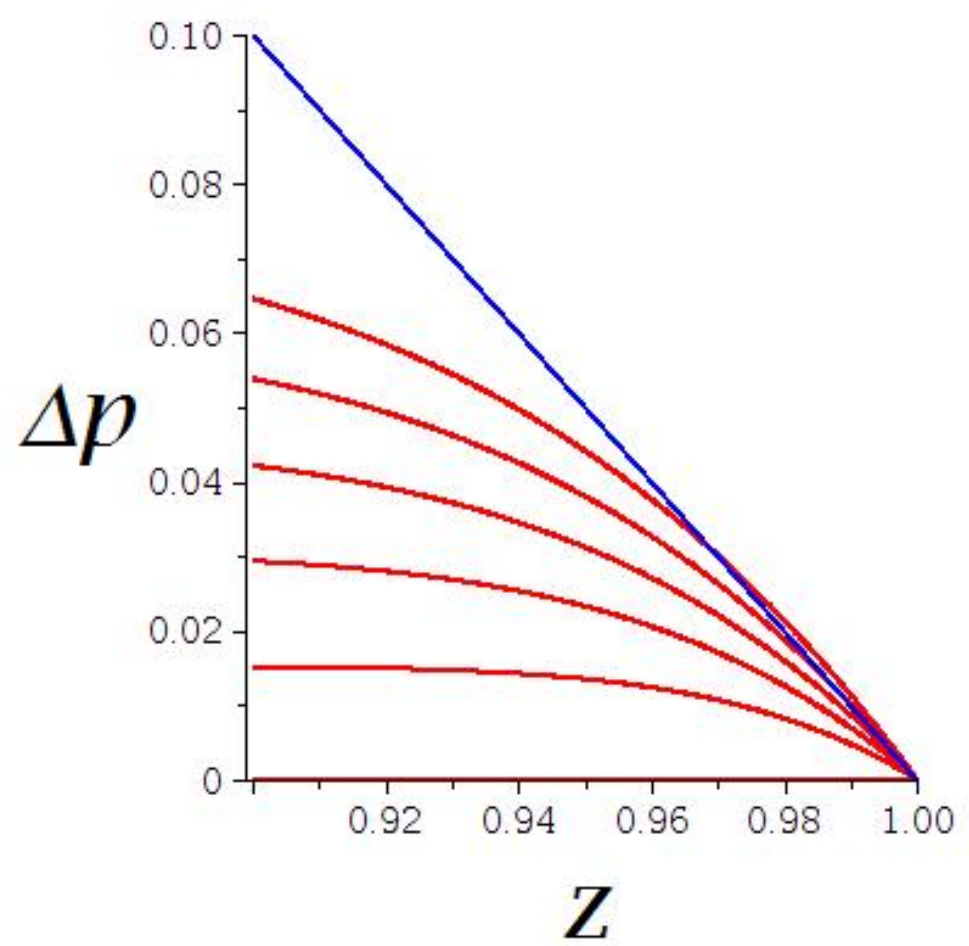

Figure 4.2: The numerical solution of equation 4.5 with the fragmentation threshold shown (blue line) where $\Delta p=p_{c}-\tilde{p}$. Here the threshold has zero tensile strength. 


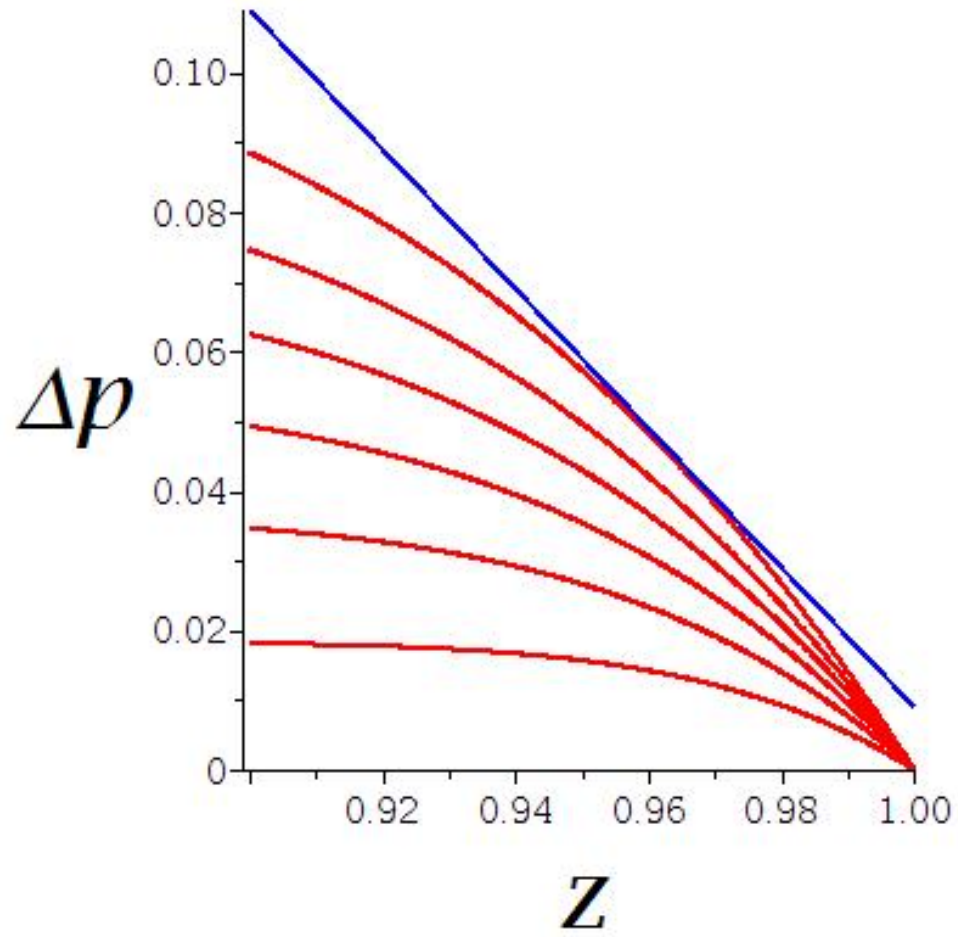

Figure 4.3: The numerical solution of equation 4.5. $\Delta p=p_{c}-p$ and the blue line shows the threshold for rupture with $S_{o}$ included. Note that $S_{o}$ raises the overburden threshold and the distance between the line and the x-axis at $z=1$ is equal to $S_{o}$. The pressure difference increases until it meets the overburden at approximately $\mathrm{t}=0.0075$, at a depth of 0.04 .

\subsection{Non-linear Diffusion}

\subsubsection{Medium-velocity case}

\subsubsection{Analytic Solutions}

When the velocity is high enough such that the $v$ and $v^{2}$ terms are comparable, the momentum conservation equation can be taken as a quadratic in $v$ and thus $v$ can be solved for. In general, we will be able to treat the $v|v|$ as $v^{2}$ as the velocity will be only in the positive $z$ direction. Thus

$$
v=\frac{\frac{-\phi^{2}}{k \phi_{o}} \pm \sqrt{\frac{\phi_{o}^{4}}{\left(k \phi_{o}\right)^{2}}+\frac{4 \phi_{o}^{4} \delta}{\sqrt{k}} p_{z}}}{\frac{2 \delta \phi_{o}^{3}}{\sqrt{k}}}
$$




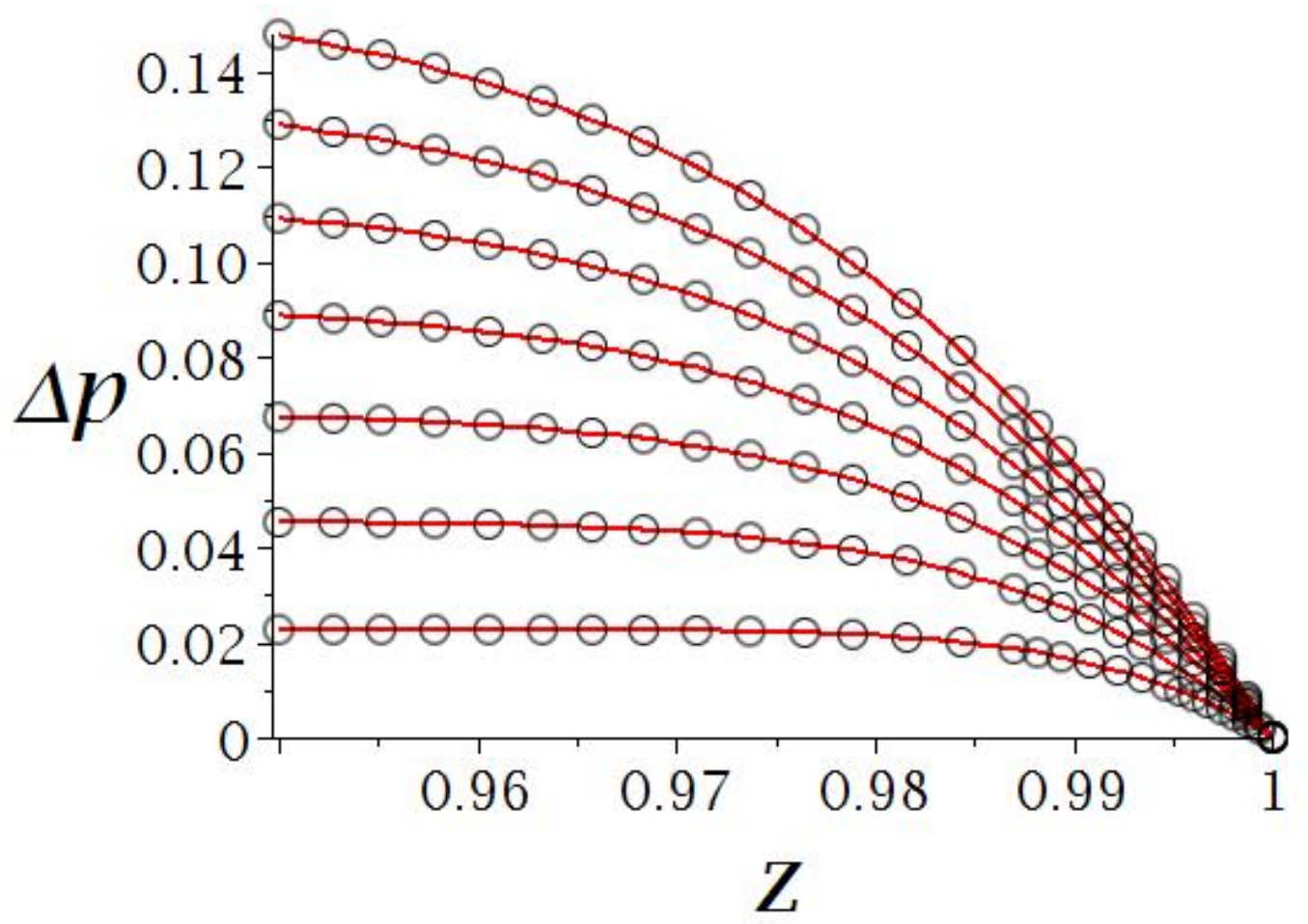

FiguRE 4.4: Comparison between the analytic solutions (circles) and numerical solutions (lines) with $\Delta p=p_{c}-p$

which using the method shown above for obtaining equation 4.5 results in the non-linear diffusion equation

$$
p_{t}+\left(\frac{\frac{-1}{k \phi_{o}}+\sqrt{\frac{1}{\left(k \phi_{o}\right)^{2}}+\frac{4 \delta}{\sqrt{k}} p_{z}}}{\frac{2 \delta \phi_{o}}{\sqrt{k}}}\right)_{z}=0
$$

which reduces to the high-velocity case below when $k$ is large and to the linear case when $\delta$ is small. We have discarded the minus term so that the equation is always non-zero. For the non-linear cases we modify the initial condition to be $p(z, 0)=\lambda z$ to avoid singularities caused by the non-linear diffusivity. We can solve equation 4.19 by separating variables $p(z, t)=p_{1}(z)+p_{2}(t)$. This splits into two equations,

$$
\frac{d^{2} p_{1}(z)}{d z^{2}}=\phi_{o} C_{1} \sqrt{\frac{\sqrt{k}-4 \delta k \phi_{o}^{2} \frac{d p_{1}(z)}{d z}}{p h i_{o}^{2} k^{\frac{3}{2}}}}
$$

and

$$
\frac{d p_{2}(t)}{d t}=C_{1}
$$


where $C_{1}$ is the separation constant. Equation 4.21 is solved by

$$
p_{2}(t)=C_{1} t+d
$$

where $d$ is a constant of integration. Equation 4.20 can be solved by completing the square under the square root and thus we obtain

$$
p_{1}(z)=-\frac{\phi_{o} C_{1}^{2} z^{3}}{3 \sqrt{k}}-\frac{\phi_{o} C_{1}^{2} C_{2} z^{2}}{\sqrt{k}}+\left(\frac{1}{4 \delta \phi_{o}^{2} \sqrt{k}}+\frac{2 \phi_{o} C_{1}^{2} C_{2}}{\sqrt{k}}\right) z+C_{3}
$$

which then gives

$$
p(z, t)=-\frac{\phi_{o} C_{1}^{2} z^{3}}{3 \sqrt{k}}-\frac{\phi_{o} C_{1}^{2} C_{2} z^{2}}{\sqrt{k}}+\left(\frac{1}{4 \delta \phi_{o}^{2} \sqrt{k}}+\frac{2 \phi_{o} C_{1}^{2} C_{2}}{\sqrt{k}}\right) z+C_{1} t+D
$$

where the arbitary constants $C_{3}$ and $d$ have been combined into $D$. While this is a solution to 4.19 , it is only of limited usefulness as will be seen below with the discussion of 4.26 .

\subsubsection{Numerical Solutions}

Solving this numerically gives Figure 4.5. It seems to show the same evolution as the linear case. This is also obvious when $\Delta p$ is plotted (Figure 4.6).

\subsubsection{High-velocity case}

\subsubsection{Analytic Solutions}

In the high velocity case we take the $v^{2}$ term to be much larger than the $v$ term in the drag equation which then becomes,

$$
D=\frac{\delta \phi^{3}}{\sqrt{k}} v|v|
$$

We can balance this with the pressure gradient term and then substitute this expression into the mass conservation equation for the gas and thus we find the non-linear diffusion equation,

$$
p_{t}+\left(\sqrt{b p_{z}}\right)_{z}
$$




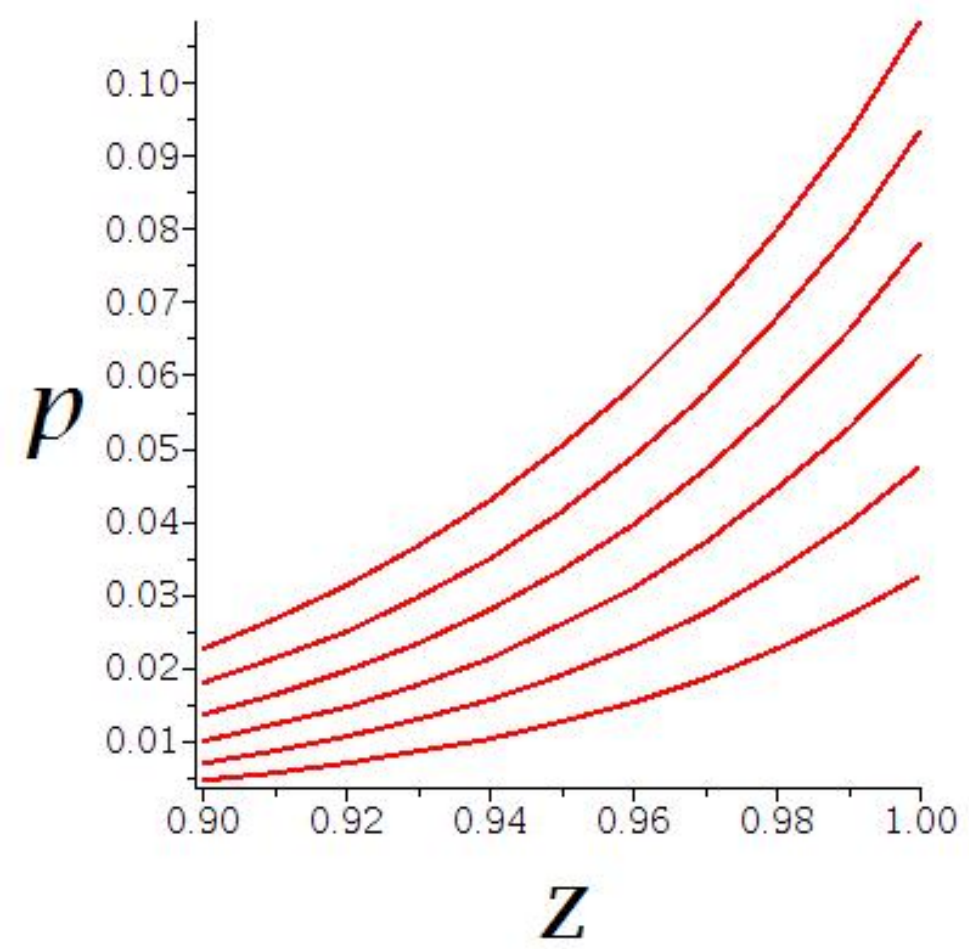

FiguRE 4.5: Numerical solution to equation 4.19 showing behavior identical to the linear diffusion case

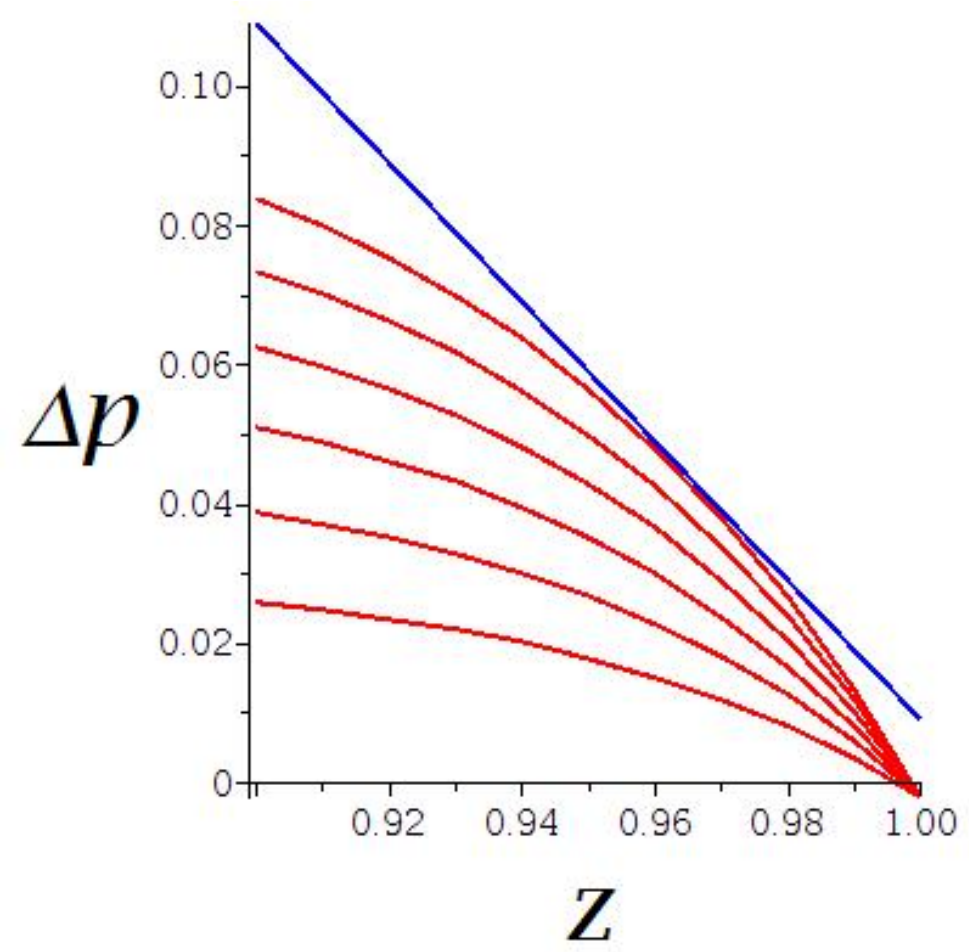

FiguRE 4.6: Numerical solution to equation 4.19 with $\Delta p=p_{c}-p$. It fragments at the same point as the linear diffusion solution though it does fragment slightly earlier (at $\mathrm{t}=0.007$ instead of $\mathrm{t}=0.0075$ ) 
where $b=\frac{\sqrt{k}}{\delta \phi^{2}}$.

This equation can be solved using separation of variables in a similar fashtion to equation 4.19 with $p(z, t)=p_{1}(z)+p_{2}(t)$. Substituting this into the equation gives

$$
\frac{d^{2}}{d z^{2}} p_{1}(z)=\frac{2 c}{b} \sqrt{b \frac{d}{d z} p_{1}(z)}, \frac{d}{d t} p_{2}(t)=c
$$

where $c$ is an arbitrary constant. A solution for $p_{2}(t)$ is

$$
p_{2}(t)=c t+d
$$

where $d$ is the constant of integration. A solution for $p_{1}(z)$ is a polynomial of form

$$
p_{1}(z)=\frac{c^{2}\left(z+d_{1}\right)^{3}}{3 b}+d_{2}
$$

thus giving

$$
p(z, t)=\frac{c^{2}\left(z+d_{1}\right)^{3}}{3 b}+c t+d_{2}
$$

This can match the exponential decay at the boundary for short time scales when $c=a$. However, matching the initial condition of $p(z, 0)=\lambda z$ cannot be done without setting $d_{1} \gg z$. This can be done by moving coordinates such that we replace $z$ with $1-\alpha$. Then when $\alpha$ is small

$$
p(\alpha, t)=\frac{a^{2}\left(d_{1}^{2} \alpha+d_{1}^{3}\right)}{3 b}+a t+d_{2}
$$

However, this still does not match the initial condition and displays odd behavior as discussed below.

\subsubsection{Numerical Solutions}

Solving the equation 4.26, using boundary and initial conditions $p_{z}(0, t)=\lambda, p(1, t)=$ $p_{c}+\lambda, p(z, 0)=\lambda z$ numerically gives the behavior shown in Figure 4.7. The $\lambda$ term has been added to avoid an initial discontinuity at $z=1$ and is $\sim 0.002$. Importantly, it shows the pressure near $z=0$ is changing at early times. This is unusual as we would expect the expansion wave to take time to penetrate into the bed but here it is starting to go down immediately. Further it shows that the analytic solutions derived earlier (Equation 4.31 could be valid for medium times, assuming a bed that has not fragmented. For long time $p$ will level out at 


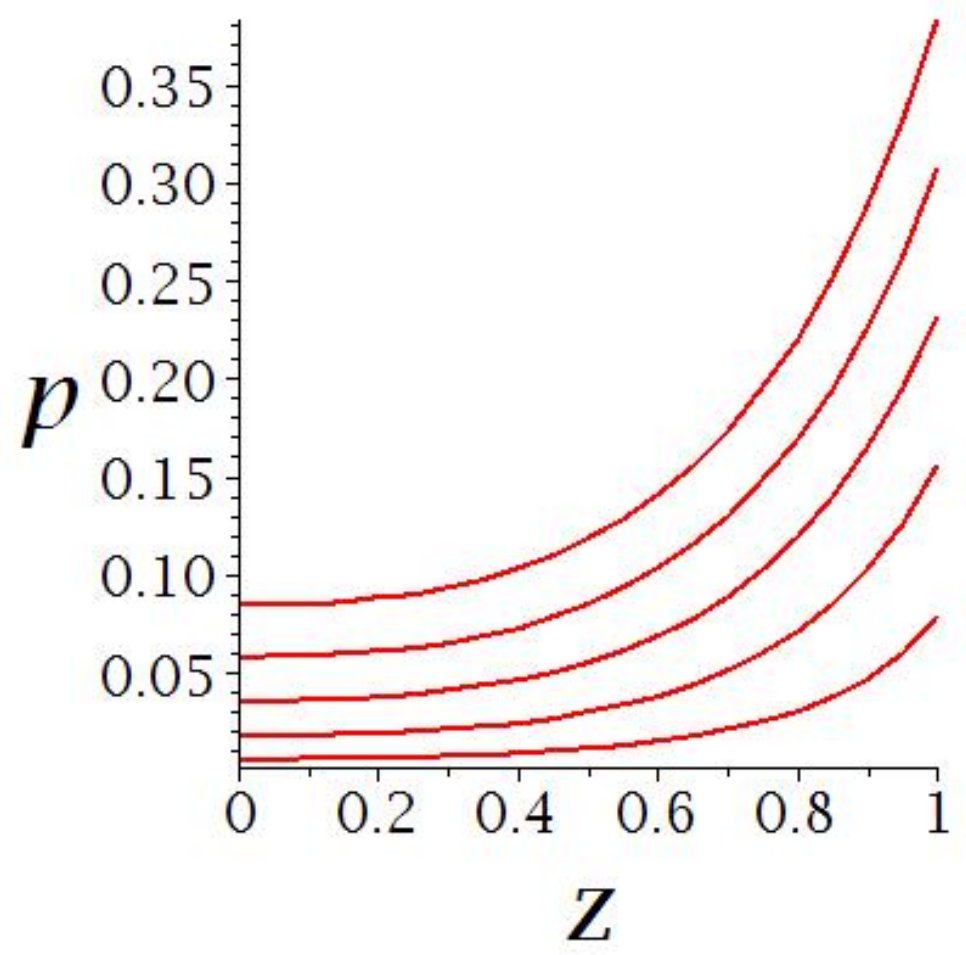

Figure 4.7: Numerical solution of high velocity diffusion equation 4.26 with time steps of 0.04 . Note the change at $z=0$ with the pressure increasing at small times.

atmospheric pressure and the analytic solution will again be invalid as it continues increases for all $t$.

For smaller times we can find the fragmentation point using similar methods as in section 4.1. Numerically this gives Figure 4.8 and has a layer size of 15 beads.

\subsubsection{Non-diffusive behavior}

A hallmark of the strange behavior shown by these solutions (Equation 4.31) is that they are 'non-diffusive'. The solutions to the non-linear cases do not show the 'spreading' characteristic of diffusion equations but rather a more 'mesa-like' behavior. This is when the diffusion causes an increase in the pressure gradient usually due to a non-linear diffusion coefficent. The pressure profiles of these equations tend to have flat areas of high diffusivity and low pressure gradients separated by areas of low diffusivity and high pressure gradients. This is what gives them their mesa-like appearance Similar behavior is also seen in the below section 5. These solutions show a steepening of the pressure near the surface 


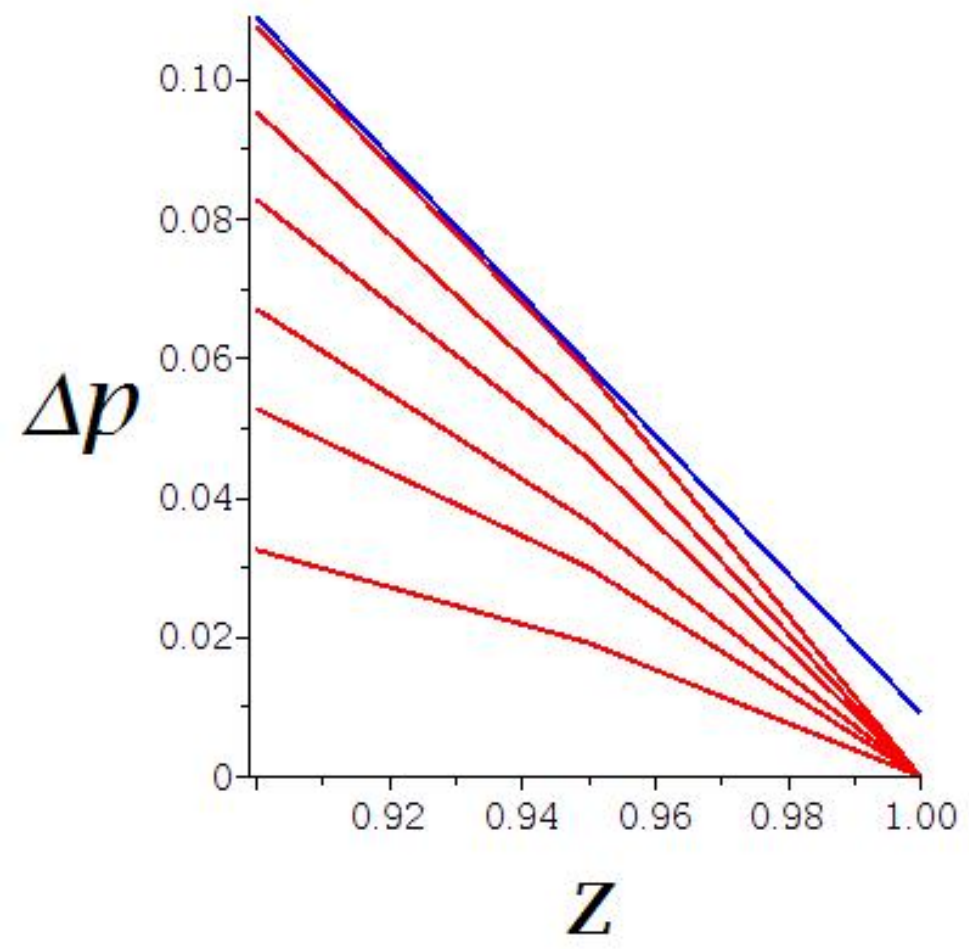

Figure 4.8: Numerical solution of high velocity diffusion with the overburden and tensile strength shown in blue. Solved at even spacings between 0 and 0.24 and fragments at $z \approx 0.95 . \Delta p=p_{c}-p$.

of the bed. Thus the maximum pressure gradient builds up and there is a nonlinear diffusivity stopping the gradient flattening as $D=\frac{1}{\sqrt{p_{z}}}$. Eventually the wave penetrates into the bed. This is similar to the modeling seen in [41] where there is modeling of a single cereal grain. The major difference in this case is that the pressure gradient, unlike the moisture gradient in the cereal grain case, only builds up to the point of fragmentation. After that it no longer builds up as it is dissipated by the movement of the slabs. A more general treatment of this behavior is found in Friedman and Höllig [42]. 


\section{Chapter 5}

\section{Wave-like Equations}

If we take $\nu=0.2 \neq 0$ ( $\nu$ is the scaling parameter for the acceleration of the gas) then we find a system of $p$ and $v$. For the case in which $\lambda$ is small and before the beads have moved $\left(u=0\right.$ and $\left.\phi=\phi_{o}\right)$ the gas momentum equation reduces to

$$
\nu \phi_{o} v_{t}=\phi_{o} p_{z}-\nu \theta_{o} C_{V M} v_{t}-\frac{\phi_{o} v}{k}-\frac{\delta \phi_{o}^{3} v^{2}}{\sqrt{k}},
$$

and the gas mass conversation equation reduces to simply

$$
p_{t}=v_{z}
$$

We can then integrate the gas mass equation with respect to time and substitute into the gas momentum equation giving.

$$
\nu^{\prime} v_{t}=\phi_{o} \int v_{z z} d t-\frac{\phi_{o} v}{k}-\frac{\delta \phi_{o}^{3} v^{2}}{\sqrt{k}},
$$

where $\nu^{\prime}=\left(\phi_{o}+\theta_{o} C_{V M}\right) \nu$. We can then differentiate with respect to time, thus giving a wave-like equation

$$
\nu^{\prime} v_{t t}=\phi_{o} v_{z z}-\frac{\phi_{o}}{k} v_{t}-\frac{2 \delta \phi_{o}^{3}}{\sqrt{k}} v v_{t}
$$

The $v^{2}$ term is small $(\sim 0.01)$ and so by setting it to zero we initially obtain,

$$
\nu^{\prime} v_{t t}=\phi_{o} v_{z z}-\frac{\phi_{o}}{k} v_{t}
$$


Pressure can be easily found due to

$$
p(z, t)=\int v_{z} d t
$$

\subsection{Analytic Solutions}

Without the $v v_{t}$ term equation 5.4 separates using $V(z, t)=T(t) Z(z)$ into,

$$
\begin{gathered}
\nu^{\prime} T^{\prime \prime}(t)+\frac{\phi_{o}}{k} T^{\prime}(t)-q T(t)=0 \\
\phi_{o} Z^{\prime \prime}(z)-q Z(z)=0
\end{gathered}
$$

where $q$ is the separation constant. We set $q=\sigma^{2}$ (as $q=0$ gives the trivial solution and $q<0$ cannot match the boundary conditions). The $z$ equation has the solution

$$
Z(z)=A_{1} \exp \left(\frac{\sigma}{\sqrt{\phi_{o}}} z\right)+A_{2} \exp \left(-\frac{\sigma}{\sqrt{\phi_{o}}} z\right)
$$

where $A_{1}$, and $A_{2}$ are arbitrary constants. The $t$ equation has the auxiliary equation

$$
\nu^{\prime} m^{2}+\frac{\phi_{o}}{k} m-\sigma^{2}=0
$$

solving for $\mathrm{m}$ gives

$$
m=\frac{1}{2 \nu^{\prime}}\left(-\frac{\phi_{o}}{k} \pm \alpha\right)
$$

where

$$
\alpha=\sqrt{\left(\frac{\phi_{o}}{k}\right)^{2}+4 \nu^{\prime} \sigma^{2}}
$$

Thus the solution for the $t$ equation is

$$
T(t)=e^{\frac{-\phi_{o} t}{2 \nu^{\prime} k}}\left(B_{1} e^{\alpha t}+B_{2} e^{-\alpha t}\right)
$$

where $B_{1}$, and $B_{2}$ are arbitrary constants. The solution to the whole problem is thus

$$
v(z, t)=e^{\frac{-\phi_{o}}{2 \nu^{\prime} k} t}\left(B_{1} e^{\alpha t}+B_{2} e^{-\alpha t}\right)\left(A_{1} \exp \left(\frac{\sigma}{\sqrt{\phi_{o}}} z\right)+A_{2} \exp \left(-\frac{\sigma}{\sqrt{\phi_{o}}} z\right)\right)
$$


Using 5.6 we can find $p(z, t)$ to be

$$
p(z, t)=b\left(\frac{B_{1}}{c+\alpha} e^{(c+\alpha) t}+\frac{B_{2}}{c-\alpha} e^{(c-\alpha) t}\right)\left(A_{1} \exp (b z)-A_{2} \exp (-b z)\right)
$$

where

$$
b=\frac{\sigma}{\sqrt{\phi_{o}}}
$$

The boundary conditions are

$$
p_{z}(0, t)=0
$$

and

$$
p(1, t)=p_{a}\left(1-e^{-a t}\right)
$$

With the initial conditon

$$
p(z, 0)=0
$$

To satisfy the boundary condition 5.17 we take

$$
p_{z}(0, t)=0=b^{2}\left(\frac{B_{1}}{c+\alpha} e^{(c+\alpha) t}+\frac{B_{2}}{c-\alpha} e^{(c-\alpha) t}\right)\left(A_{1}+A_{2}\right)
$$

which is zero for all $t$ as long as $A_{1}=-A_{2}$. To satisfy the initial condition 5.19 we take

$$
p(z, 0)=0=b\left(\frac{B_{1}}{c+\alpha}+\frac{B_{2}}{c-\alpha}\right)\left(A_{1} e^{b z}+A_{1} e^{b z}\right)
$$

This holds for all $z$ if

$$
\frac{B_{1}}{c+\alpha}=-\frac{B_{2}}{c-\alpha}
$$

The last boundary condition 5.18 gives

$$
p(1, t)=p_{a}\left(1-e^{-a t}\right)=b\left(\frac{B_{1}}{c-\alpha}\left(e^{(c+\alpha) t}+\frac{B_{2}}{c-\alpha} e^{(c-\alpha) t}\right)\left(A\left(e^{b}-e^{-b}\right)\right)\right.
$$

To match this, we must take $c-\alpha=-a$ and $c+\alpha=0$. However, this results in a divergence in the coefficient $\frac{B_{1}}{c+\alpha}$. To avoid this we set $B_{1}=0$ but require

$$
\frac{B_{1}}{c+\alpha}=-\frac{B_{2}}{c-\alpha}=C
$$

, where

$$
C=b p_{a} A
$$


Solving for $\alpha$ in the simultaneous equation gives $2 \alpha=a$. Because $\alpha$ is a function of $\sigma$ from(5.12), this gives $\sigma$

$$
\sigma^{2}=\frac{1}{4 \nu^{\prime}}\left(a^{2}-\frac{2 \phi_{o} a}{2 \nu^{\prime} k}+\frac{\phi_{o}^{2}}{4 \nu^{\prime} k}-\frac{\phi_{o}^{2}}{k}\right)
$$

However, this is less than zero, we end up with imaginary pressures. As we know that the pressures are real we set the last free parameter $A$ to be $-i$. The spatial dependence becomes

$$
2 \sin (-i b z)
$$

Thus we obtain the solution

$$
p(z, t)=-i b p_{a}\left(1-e^{-a t}\right)\left(e^{b z}-e^{-b z}\right)
$$

Note that because $b$ is purely imaginary $i b$ is purely real.

\subsection{Numerical Solutions}

Solving the problem numerically shows that there is a penetrating wave. The pressure is piecewise linear between 0 and $p_{c}$. This can be easily modelled using a piecewise linear function which is found by assuming a constant gradient and setting the line to follow the boundary condition at $z=1$. This gives:

$$
p=\left\{\begin{array}{lc}
0 & 0<z<1-\hat{z} \\
11.25 z-11.25+p_{c} & 1-\hat{z} \leq z<1
\end{array}\right.
$$

Where $\hat{z}=1+\frac{p_{c}}{11.25}$ This provides a good correspondence to the numerical solution for small $t$. However, it diverges for larger $t$. Further the divergence is closer to $\hat{z}$ due to the fact that the other end is set to be equal to the boundary condition $p(1, t)=p_{c}$. When this solution is used to find the fragmentation point the pressure difference rises up to meet the overburden in the same manner as in the linear case, but the pressure difference meets the overburden at the inflection point. This corresponds to $\hat{z}$ in the piecewise solution.

When we include the $v^{2}$ term we find that the numerical solution has significant oscillation around $\hat{z}$ as seen. 


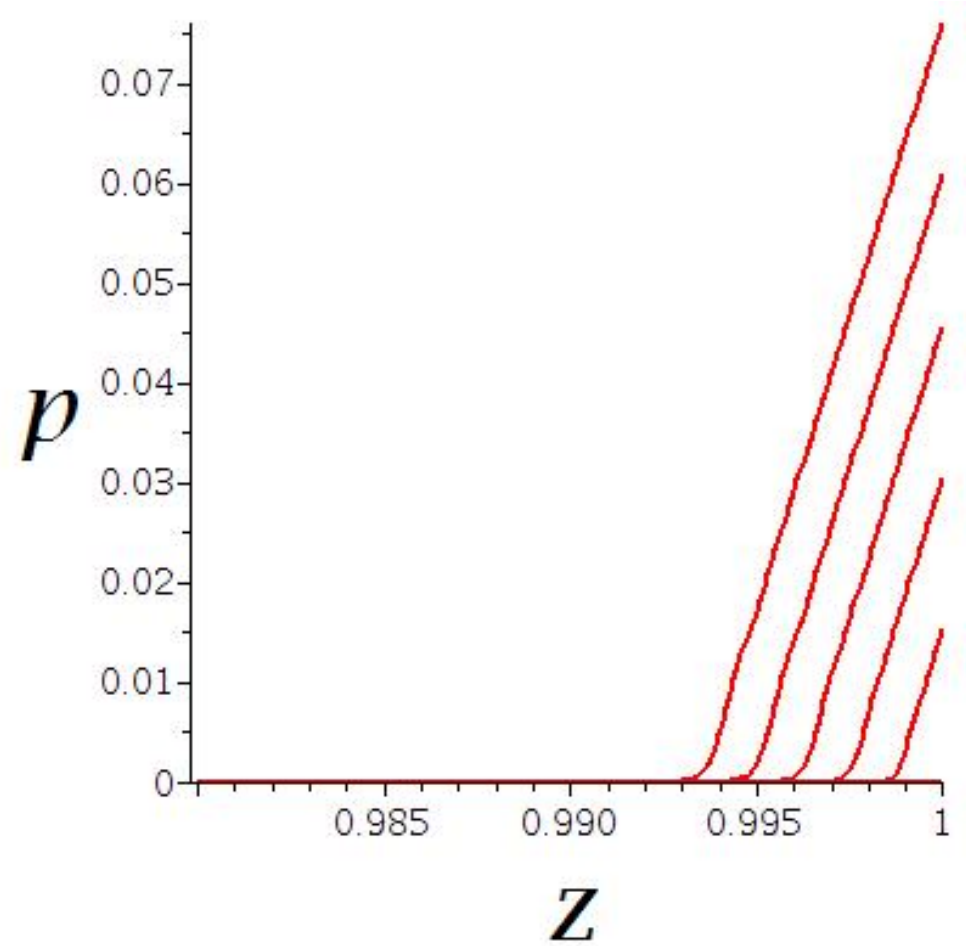

FIGURE 5.1: Numerical solution of equation 5.5 showing penetrating wave behavior

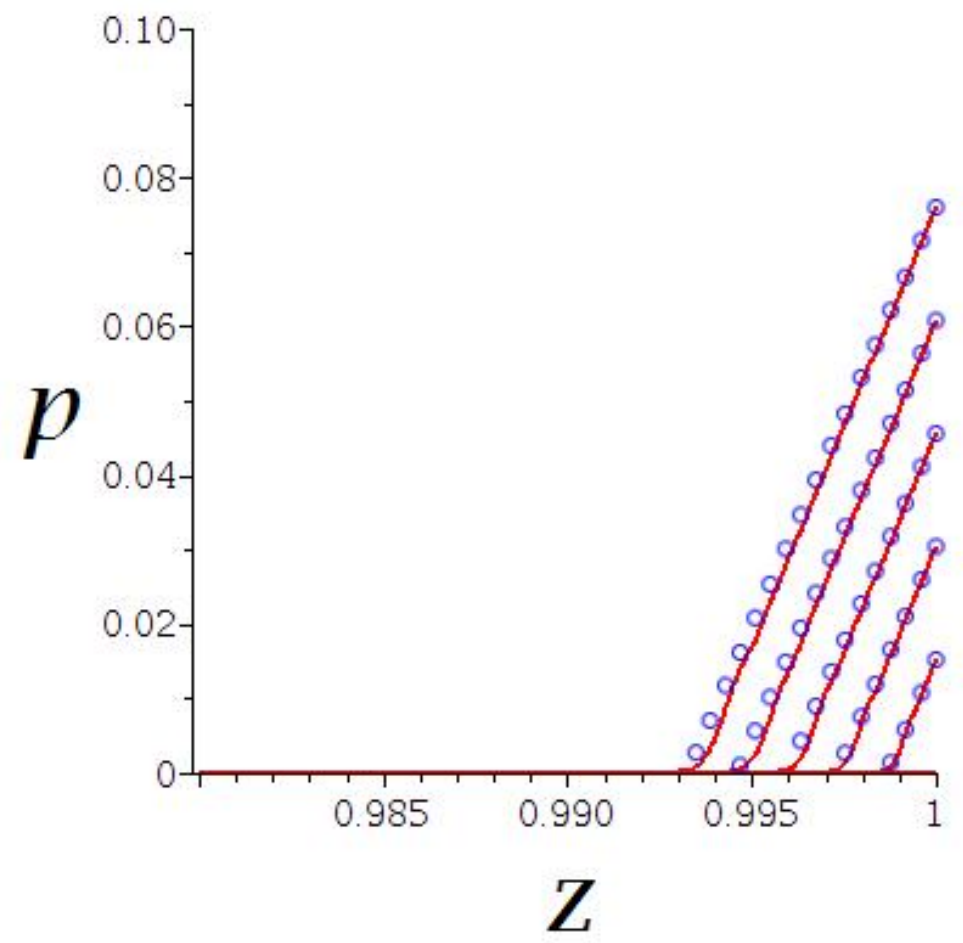

Figure 5.2: Comparison of the piece linear solution (blue circles) to the numerical solution 


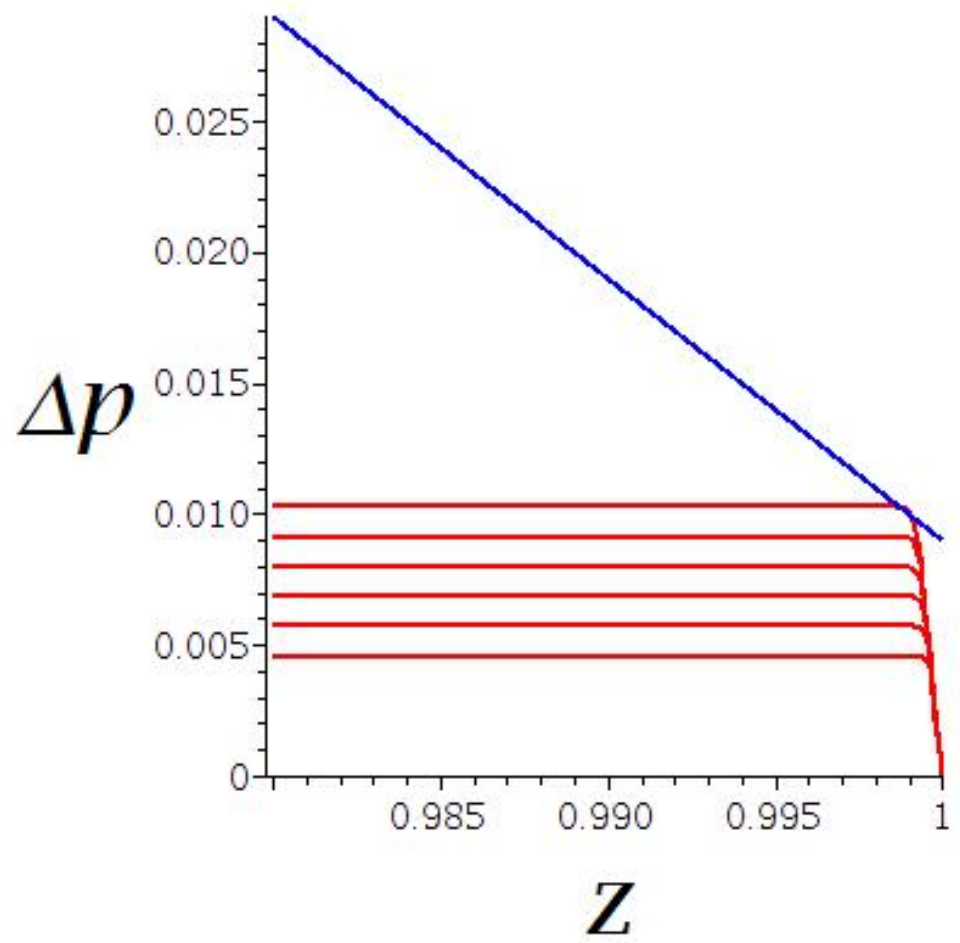

FiguRe 5.3: Numerical solution of equation 5.5 with $\Delta p=p_{c}-p$

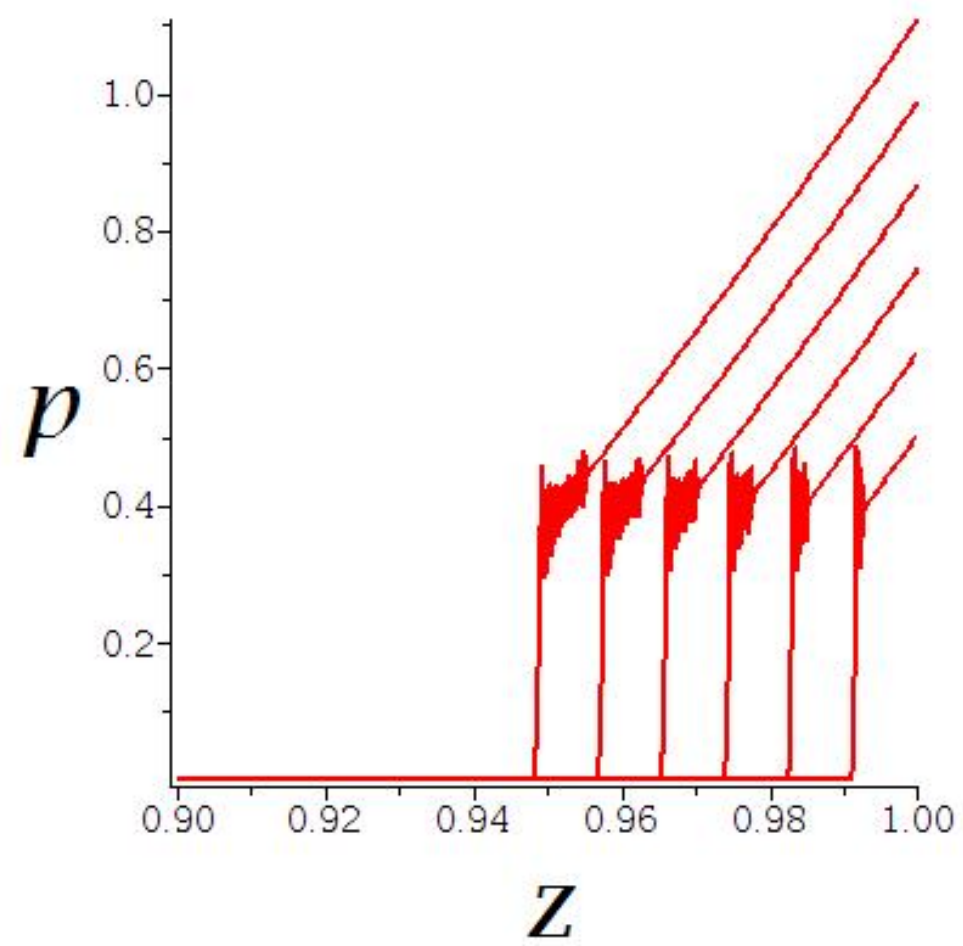

FiguRE 5.4: Numerical solution of the system 5.1 and 5.2. 


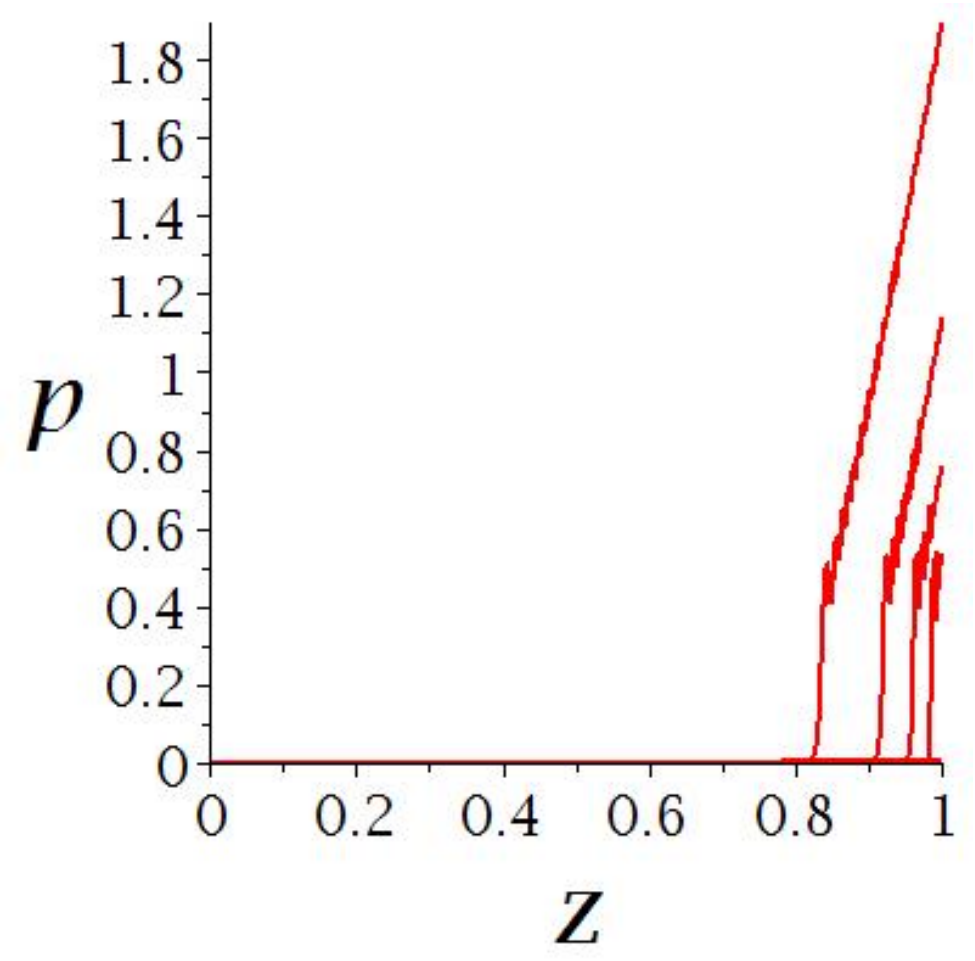

FiguRE 5.5: Numerical solution of the system with $\lambda$ non-zero.

For the case where $\lambda$ is non-zero, the numerical solution can be obtained for the system:

$$
\begin{gathered}
\nu \phi_{o}(1-\lambda p) v_{t}=\phi_{o} p_{t}-\nu\left(\frac{\theta}{2}(1-\lambda p) v_{t}\right)-\phi v-\delta \phi^{3}\left((1-\lambda p) v^{2}\right) \\
\frac{\partial p}{\partial t}=\frac{\partial}{\partial z}((1-\lambda p) v)
\end{gathered}
$$

The numerical solution shows small oscillations beyond $\hat{z}$ which is likely an artifact of the method used by MAPLE to solve the system. This is due to the osscilation frequency being inversely proportional to the spatial step size. This is seen in both Figure 5.4 and Figure 5.5. 


\section{Chapter 6}

\section{Tensile Strength and Hoop Stress}

\subsection{Tensile Strength}

Note that the variables in this section are dimensional. Before this we have taken the tensile strength of the bed to be a constant $S_{o}$ and dependent purely on $\rho_{s}, g$ and $D_{p}$, but this can take several different forms. We can take

$$
S_{o}=G+\rho g \frac{D_{p}}{2}
$$

where $\mathrm{G}$ is the additional cohesion due to Van der Waals interactions. Molerus [43] breaks the cohesive force into

$$
H=H_{o}+\kappa N
$$

where $H_{o}$ is the intrinsic cohesion caused mostly by van der Waals' forces and $N$ is the compressive normal force. Molerus finds $H_{o}$ to be

$$
H_{o} \approx \frac{\hbar \bar{\omega}}{8 \pi z_{o}^{2}} R^{*}\left(1+\frac{\hbar \bar{\omega}}{8 \pi^{2} z_{o}^{3} H^{*}}\right)
$$

where $\hbar \bar{\omega}$ is the Lifschitz-van der Waals constant (in eV), $z_{o} \approx 4 \times 10^{-10}$ is the maximum adhesion distance (in $\mathrm{m}$ ), $H^{*}$ the hardness of the solid (in $\mathrm{N} \mathrm{m}^{-2}$ ) which is $\sim 10^{8}$ in glass beads, and $R^{*}$ which is the effective particle diameter taking into account that the surfaces are not smooth (in $\mathrm{m}$ ). Molerus finds that this results in an $H_{o}$ of $8.76 \times 10^{-8} \mathrm{~N}$ in glass. Converting into pressures gives $\sim 0.028 \mathrm{~Pa}$, significantly smaller than the gravitational overburden $(\sim 1$ near the surface). 
The compressive normal force ( $\mathrm{N}$ in 6.2) is highly dependent on the rate at which the cylinder is charged with nitrogen. Note that this is not the current force on the spheres but the force applied to the spheres during the charging. The force applied to the spheres would be proportional to the pressure difference between the top of the chamber and the pore space of the bed. To estimate the size of this effect we take a pressure $p_{1}$ averaged from the top of the bed to the top of the chamber, being increased isothermally at a constant rate $R_{1}$ and bleeding into the bed at a rate $R_{2}\left(p_{1}-p_{2}\right)$ which we treat as a second chamber with average pressure $p_{2}$. Conservation of gas mass gives two differential equations:

$$
\begin{gathered}
\frac{d p_{1}}{d t}=R_{1}-R_{2}\left(p_{1}-p_{2}\right), \\
\frac{d p_{2}}{d t}=R_{2}\left(p_{1}-p_{2}\right) .
\end{gathered}
$$

Note that we have assumed that $V_{1} \sim V_{2}$ where $V_{1}$ is the volume of the top of the chamber and $V_{2}$ is the volume of the pore space of the bed. This allows the use of pressure instead of gas mass through the ideal gas law $P V \sim m T$ where in this case $m$ is the gas mass. The result does not significantly change if the volumes are different. Further, we take the initial pressures $p_{1}(0), p_{2}(0)$ to be equal to atmospheric pressure. Subtracting the second equation from the first and substituting $\Delta p=p_{1}-p_{2}$ gives the following equation

$$
\frac{d \Delta p}{d t}=R_{1}-2 R_{2} \Delta p
$$

where the initial condition becomes $\Delta p=0$. The solution to 6.6 is

$$
\Delta p=\frac{R_{1}}{2 R_{2}}\left(1-e^{-2 R_{2} t}\right)
$$

which starts at zero and approaches the asymptote $\frac{R_{1}}{2 R_{2}}$. The asymptotic value will be used as a proxy to find the compressive force from 6.2. To estimate $R_{2}$ we note that complete discharge in Anilkumar's experiments take approximately $40 \mathrm{~ms}$, giving $R_{2} \approx 25 \mathrm{~s}^{-1}$. If the top part of the chamber is charged slowly over 10 minutes for a total charge of $200 \mathrm{kPa}, R_{1}=333 \mathrm{~Pa} \cdot \mathrm{s}^{-1}$. The force on the bed from the charging time is $\sim 13 \mathrm{~Pa}$. On the other hand if the bed is charged over two seconds we find that the compressive force is 2000Pa. Thus the compressive force is highly dependent on the experimental conditions.

The variance of the tensile strength with particle diameter depends on several 
competing factors, two of which are: van der Waals' forces getting weaker as the beads get larger and the contact area per total area between the spheres also gets smaller. This is in contrast to the experimental result showing that the layer size becomes larger as bead diameter increases which would imply a higher tensile strength according to our results so far.

Further, we have not considered the packing process. We do not know if the beads had retained any static charge during the packing process which is likely to create a great deal of extra tensile strength. Nevertheless, for a given measured charge it is relatively simple to calculate the extra adhesion using Coulomb's law. A further consideration is moisture in the chamber during the experiment. This would likely result in bridges forming between the beads causing the effective contact area to increase greatly. Some of the effects of this are modeled in Groger [44].

\subsection{Hoop Stress}

One thing that has been excluded from the analysis so far is friction from the walls of the container. This "hoop stress" [45] can be derived using a force balance equation as so; the force acting on a thin layer of beads is the solid pressure difference across the layer times the area of the layer $\pi R^{2} \frac{\partial P_{s}}{\partial z} d z$ where $\mathrm{R}$ is the radius of the container. This force is balanced by gravity acting on the beads $\pi R^{2} \rho_{s} g d z$ and the friction of the wall $2 \pi R \mu P_{s} d z$ where $\mu$ is the coefficient of friction. $\mu$ is known to vary between 0.31 and 0.39 according to Malla 2007 [46]. While here we have taken the container to be circular, the geometry of the container does not change the analysis. This is pressure dependent though we take it to be a constant for numerical purposes. It is unknown how this will vary with particle diameter though some possibilities are discussed below. This gives the differential equation:

$$
\frac{\partial P_{s}}{\partial z}+\frac{2 \mu}{R} P_{s}+\rho_{s} g=0
$$

We assume that the solid pressure at the top of the bed $(\mathrm{z}=\mathrm{l})$ is 0 giving the required boundary condition. We can take an exponential solution of the form

$$
P_{s}(z)=A+B e^{\alpha z}
$$


Substituting it into the differential equation gives

$$
\alpha B e^{\alpha z}+\rho_{s} g+\frac{2 \mu A}{R}+\frac{2 \mu B}{R} e^{\alpha z}=0
$$

Now by matching coeffcients gives

$$
\begin{gathered}
A=-\frac{\rho_{s} g R}{2 \mu} \\
\alpha=-\frac{2 \mu}{R}
\end{gathered}
$$

Now, adding the boundary conditions gives

$$
B=\frac{\rho_{s} g R}{2 \mu} e^{\frac{2 \mu}{R}}
$$

Thus giving the solution

$$
P_{s}(z)=\frac{\rho_{s} g R}{2 \mu}\left(e^{\frac{2 \mu}{R}(l-z)}-1\right)
$$

We have interpreted $P_{s}$ as the fragmentation criterion to replace the overburden. Thus the pressure difference must overcome $P_{s}+S_{o}$ to cause a layer to lift off. Note that for $\mu \ll 1$ this reduces to the overburden from gravity alone used earlier.

The leading term is approximately $300 \mathrm{~Pa}$, significantly higher than the previous estimate of the overburden given by inter-particle interactions of approximately $150 \mathrm{~Pa}$. However, the function reduces to zero at the surface (by the boundary condition).

While here we have taken the container to be circular, the geometry of the container does not change the analysis, with the $\mathrm{R}$ term being replaced with the ratio of the area and the perimeter. But it is highly dependent on the radius of the container and so has a very different length scale compared to the tensile strength. Using this as the fracture criterion we see that the bed fractures at approximately $z \approx 0.96$ which corresponds to 12 bead layers, giving an excellent match to the experimental results.

The applicability of the hoop stress model to the larger context of large chemical reactors or volcanic eruptions is unknown as the stress is highly dependent on $R$ which will be tens to hundreds of meters in the larger cases which will be $10^{5}$ times the pressures seen here. 


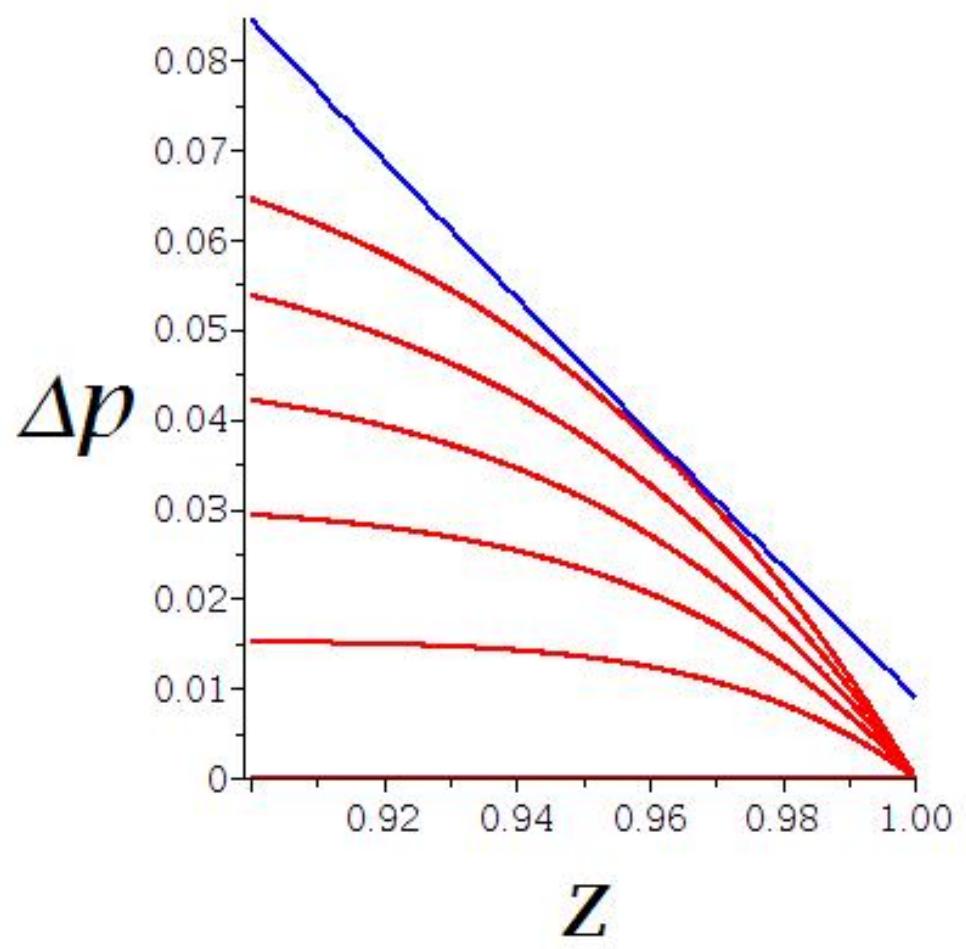

FiguRE 6.1: Solution to linear diffusion equation using the hoop stress fracture criterion. The pressure difference meets the restraining force at $z=\approx 0.96$ at $t \approx 0.005$. 


\section{Chapter 7}

\section{Dependence on Bead Diameter and Density}

\subsection{Dependence on particle diameter}

So far, we have only considered the behavior of the bed of bead diameter $D_{p} \approx$ $500 \mu \mathrm{m}$. Both the tensile strength and the permeability depend on the bead diameter and so we must consider how this affects our results. If the bead diameter increases but the density of the beads stays the same then the inherent tensile strength of the beads $S_{o}$ increases linearly. Moreover, the permeability of the bed will increase as the square of the bead diameter. Thus for beads of $1000 \mu \mathrm{m}$ the rescaled permeability is 4 and we can solve the problem again to find the graph below. Conversely, if the bead diameter is $125 \mu \mathrm{m}$ then the tensile strength is much lower and the permeability becomes $\frac{1}{16}$. By working out the fragmentation point, we can see that the slab thickness is directly proportional to the bead diameter (Figure 7.3) and is proportional to the square root of the bead diameter when expressed in terms of particle number (Figure 7.4).

Comparing to experiment we see that the model underestimates the slab size at small bead diameters but matches well at larger sizes. A possible cause is an extra source of cohesion that is only significant at small bead diameters such as $H_{o}$. However, it does approach zero layer size at small bead diameters which matches the experiments by Cagnoli et al [29]. 


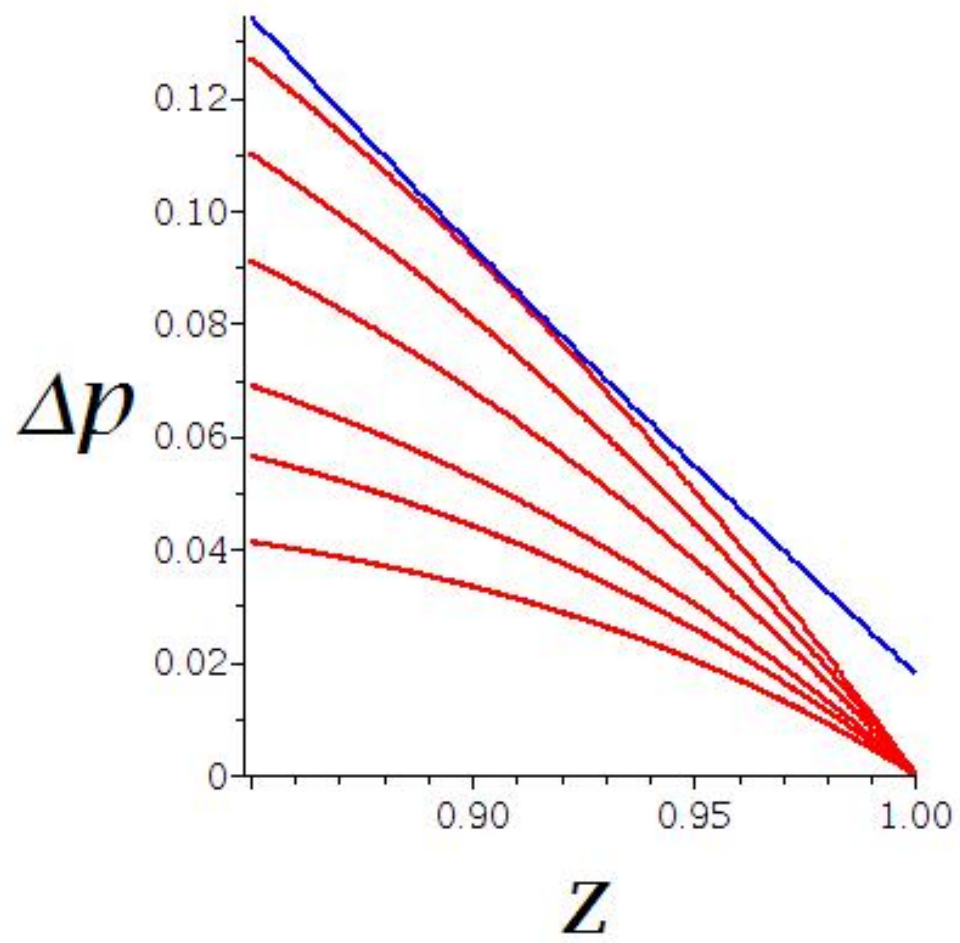

Figure 7.1: Solution to linear diffusion equation 4.5 with $D_{p}=1000 \mu m$. The blue line know includes the hoop stress as well as the overburden and $S_{o}$.

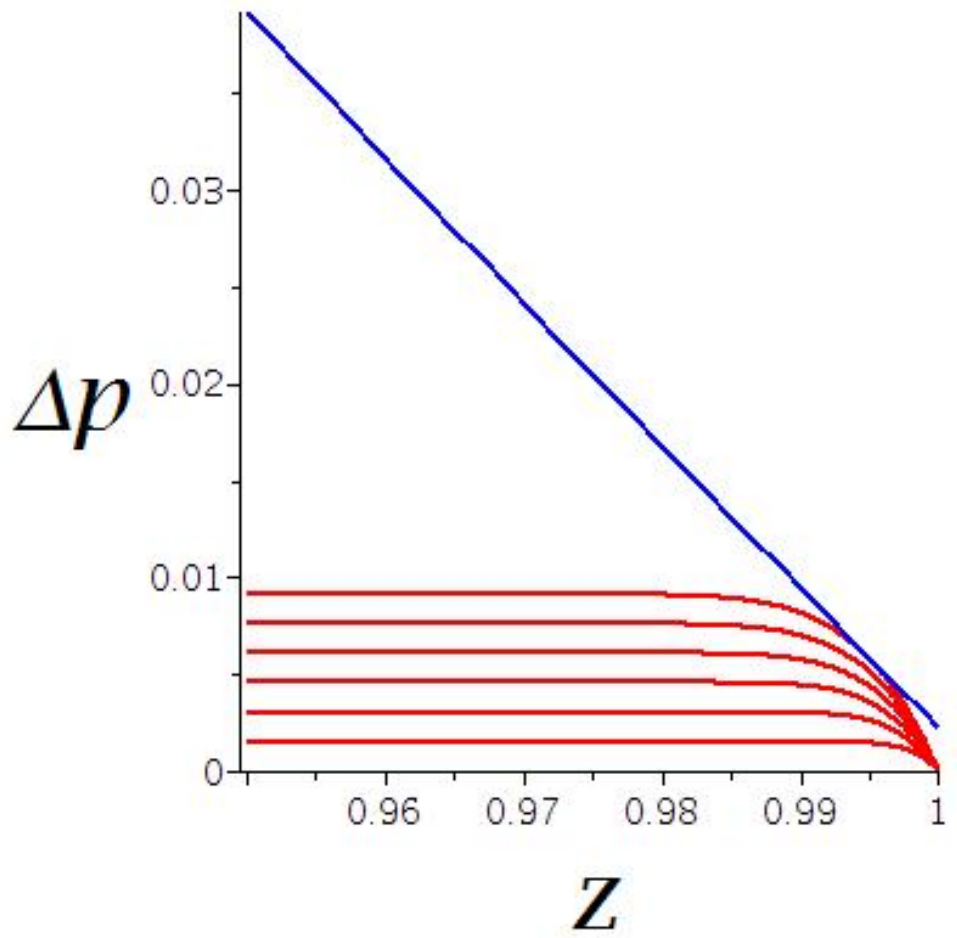

FiguRE 7.2: Solution to linear diffusion equation 4.5 with $D_{p}=125 \mu \mathrm{m}$. Fragmentation is very early $(\mathrm{t}=0.0005)$ and at a depth of $\mathrm{z}=0.005$. 


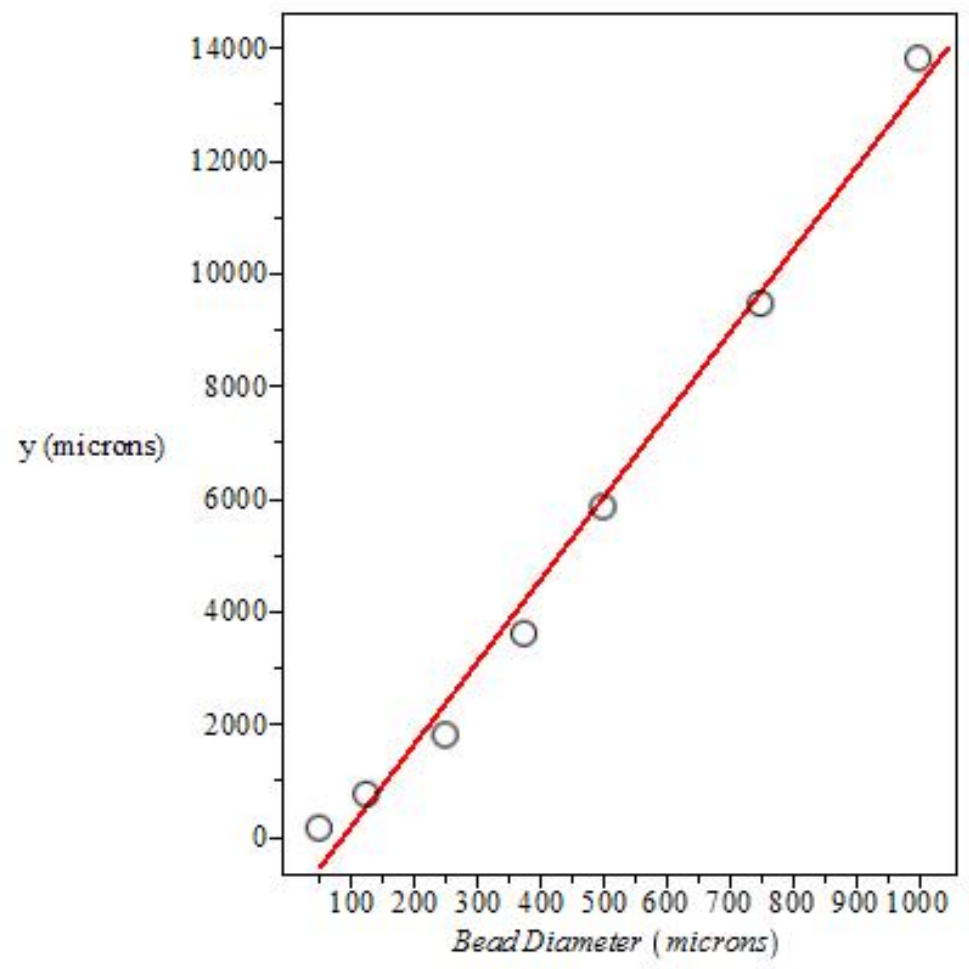

Figure 7.3: Slab size (y) dependence on bead diameter in terms of microns showing the linear dependence

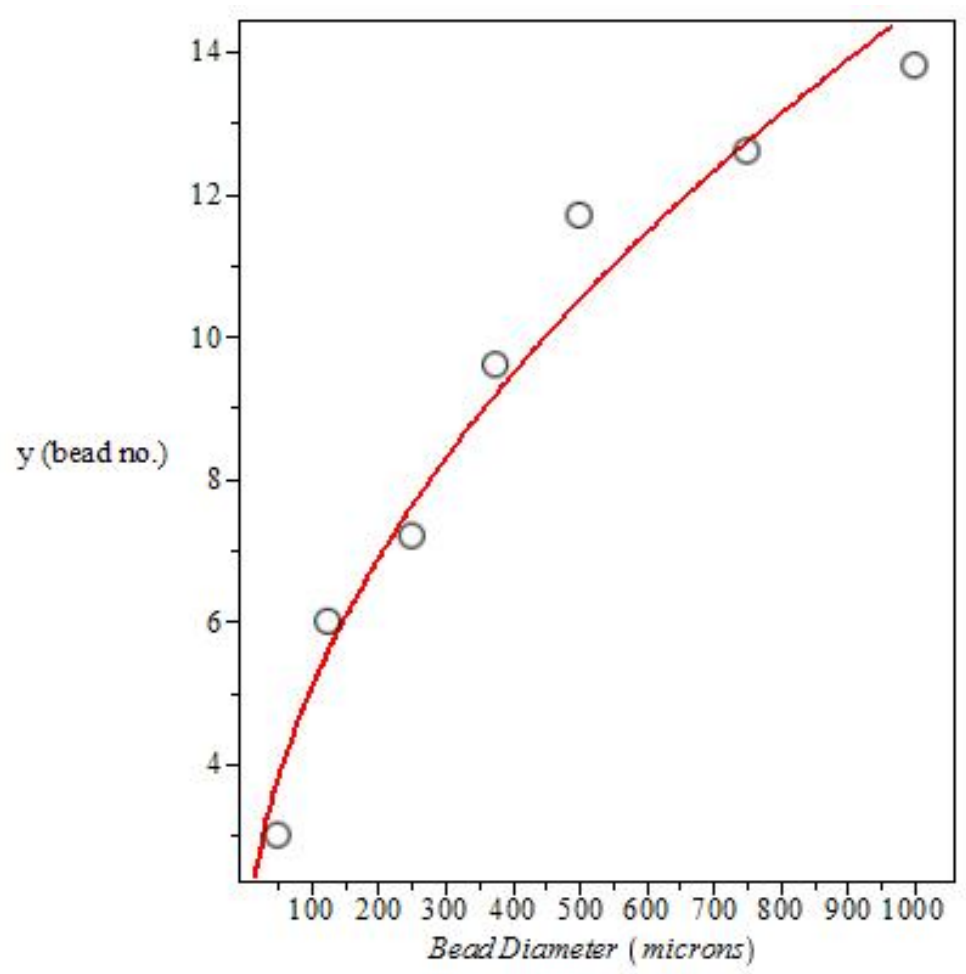

Figure 7.4: Slab size (y) dependence on bead diameter in terms of bead number showing a square root dependence 


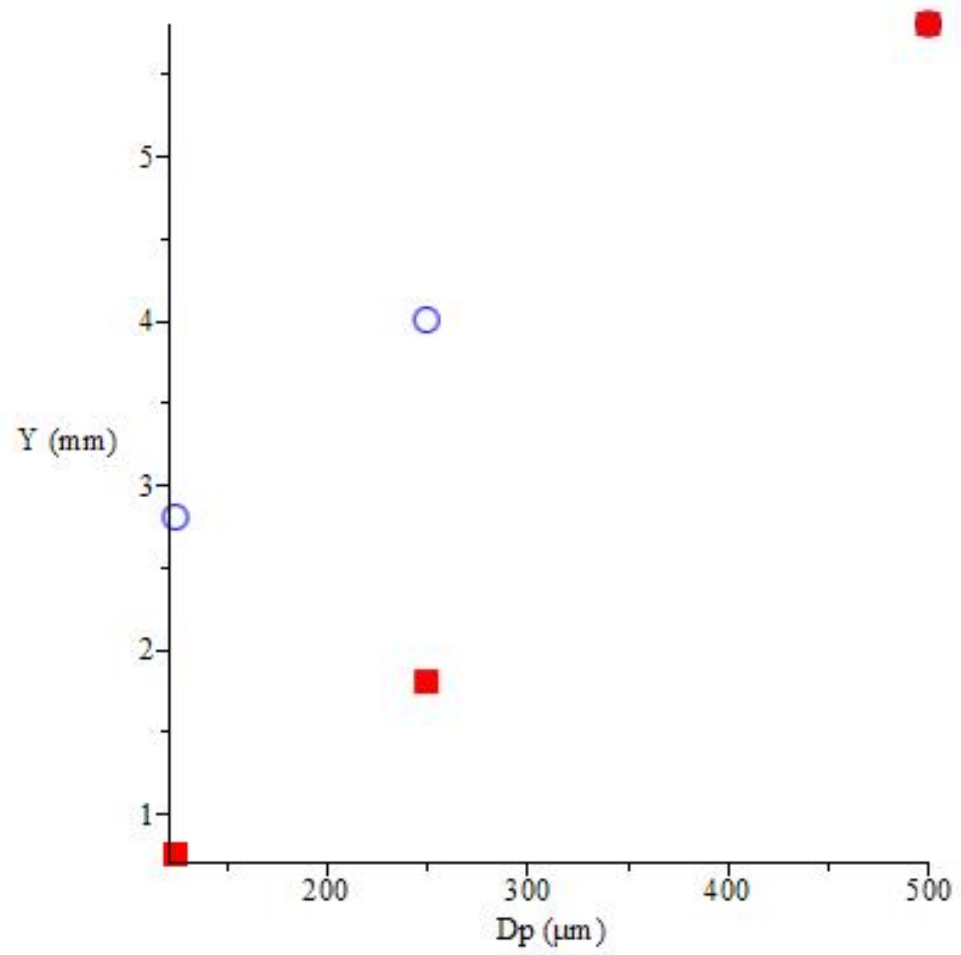

Figure 7.5: A comparison of the experimental data for the slab size from Anilkumar [28] and modeled slab size, showing a divergence at small bead diameters and a very good match at $500 \mu \mathrm{m}$.

\subsection{Dependence on bead density}

Increasing the bead density increases both $S_{o}=\rho g \frac{D_{p}}{2}$ and the hoop stress and this results in the slab size increasing approximately linearly until $5000 \mathrm{~kg} \mathrm{~m}^{-3}$. After that it levels off. However, even though the fragmentation point stops going deeper (Figure 7.6), the fragmentation time does increase as shown below (Figure 7.7 . 


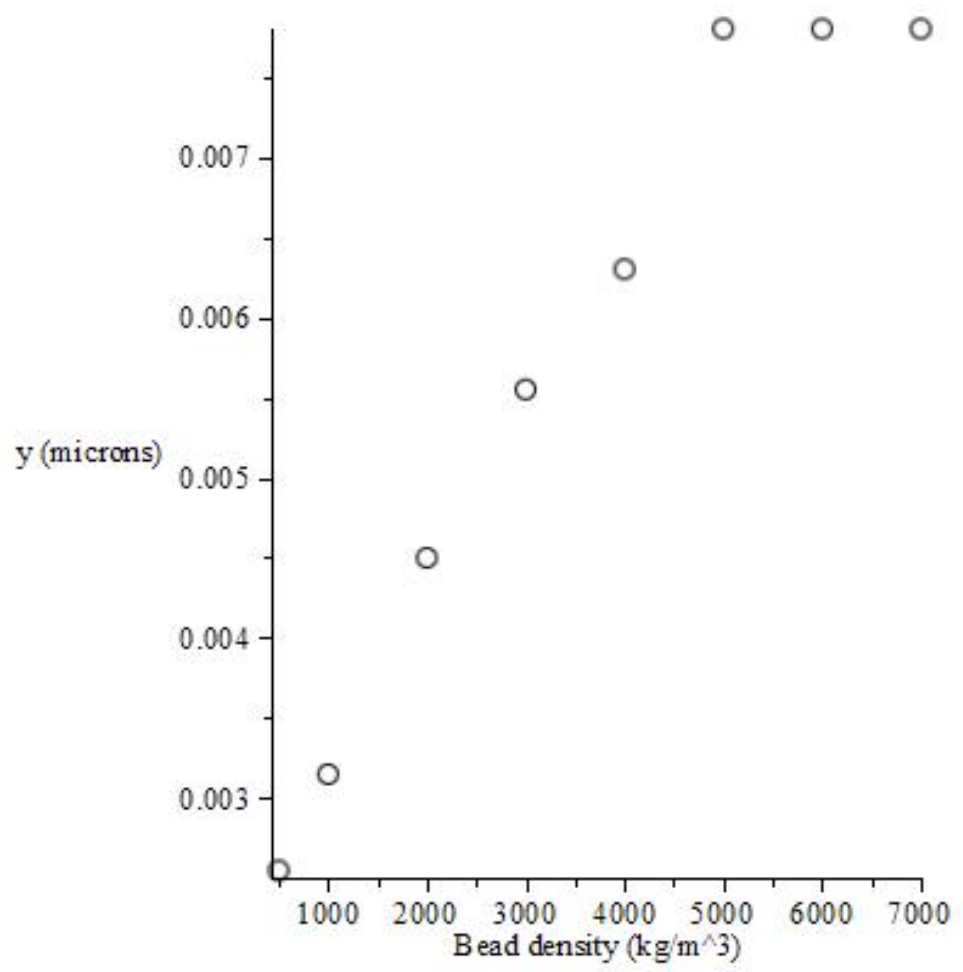

FiguRE 7.6: Slab size (y) dependence on bead density in microns showing a leveling off above $5000 \mathrm{~kg} \mathrm{~m}^{-3}$

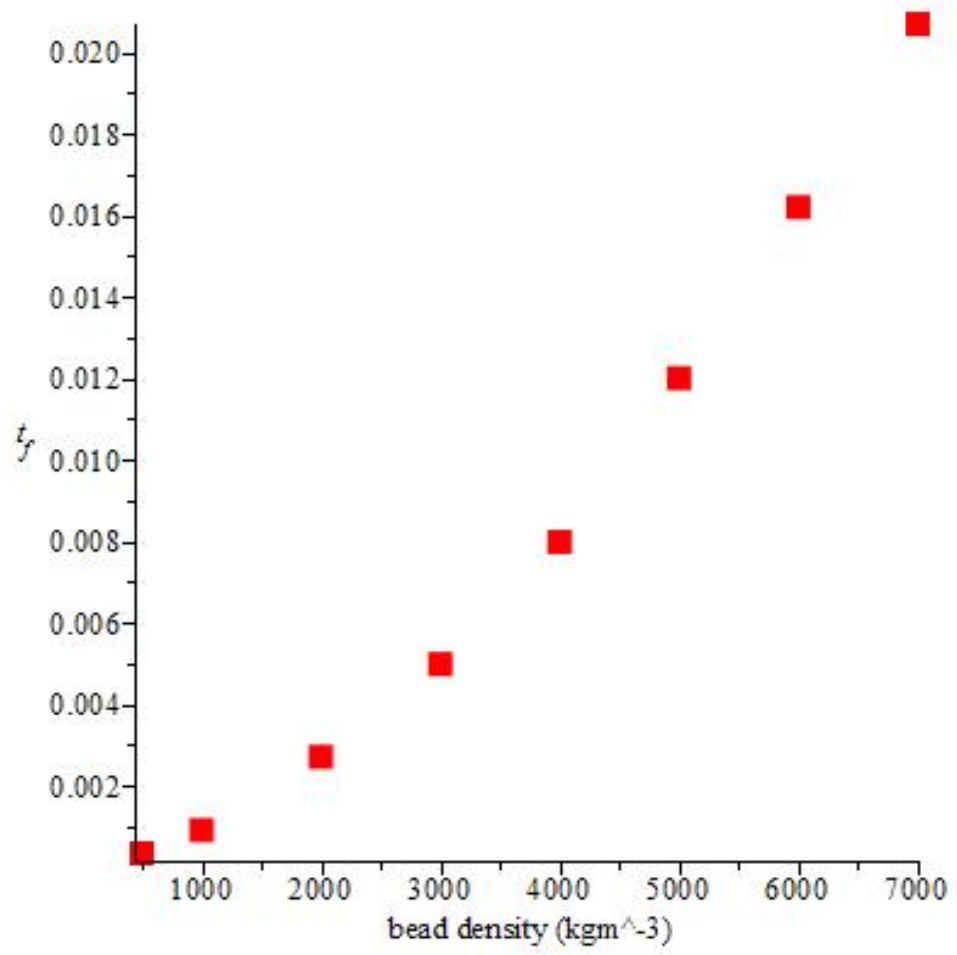

Figure 7.7: Dependence of fragmentation time on bead density. $t_{f}$ is in dimensionless units. It does not show the leveling off seen in the fragmentation point 


\section{Chapter 8}

\section{Conclusion}

We have modeled and explained why the sudden depressurization that occurs in shock tube experiments results in the formation of layers (slabs), by deriving, reducing and solving a mathematical model consisting of conservation equations for mass and momentum for gas and a porous solid with low tensile strength, with the gas flowing due to an adiabatic expansion above the bed. The effects of gravity and wall friction have been included as a criterion for fragmentation.

The model reduces to a linear diffusion equation, solved using Laplace transforms and numerically. Cases of medium to high velocity and large gas accelerations have also been explored and solved analytically and numerically. The numerical solution of the large acceleration case has also been shown to have a good fit with a piecewise linear function.

The expansion wave travels down from the head of the chamber and when reaching the bed causes a pressure drop that had been experimentally determined. The expansion wave then diffuses into the bed and builds up a pressure gradient until that pressure gradient overcomes the gravitational overburden, the inherent tensile strength of the bed, and the wall friction. The layer then lifts off and accelerates upwards. The expansion wave will continue downwards with the remaining part of the bed behaving in the same fashion as each layer lifting off results in a new uncovered bed that is smaller but otherwise identical. This process continue with layers being formed all the way down the bed. We used a hoop stress formulation for the wall friction which is not generalisable to layered beds. The dependence of layer size on bead diameter was also investigated using the numerical solutions of the linear diffusion with the hoop stress formulation added. Here we found a good 
match to experimental results, particularly at larger bead diameters. The dependence of layer size on bead density was also investigated to provide predictions for future experiments. It was found that the layer size increases to a maximum at $5000 \mathrm{~kg} \cdot \mathrm{m}^{-} 3$ before leveling off. However, it was found that the fragmentation time continues to increase even after the leveling off.

There are several ways this model could be extended. They include: modeling the movement of the bed after fragmentation, modeling friction and cohesion in a more intrinsic fashion, and modeling layered beds. More experimental data would also allow closer modeling and comparison to the predicted effect of changes in bead density.

In this work we have ignored what happens to the bed after fragmentation. After the bed fragments, porosity is no longer a constant and the bed velocity cannot be taken to be zero. This would result in significant complexity and it is likely that it can only be modelled numerically as was seen in the equivalent modeling in rocks [32]. Further, the modeling used here is quasi-static which may not apply after fragmentation.

The model of friction used in this work is an ad hoc addition to the cohesion. While this results in the model matching the experiments, it runs into problems when modeling layered beds. Similarly, the model of cohesion as $S_{o}=f\left(\rho_{s}, D_{p}\right)$, while acceptable on the macro level, is likely to break down at the bead level, which again may cause problems with layered beds. A possible candidate for this is to add a stress-strain relation and add friction to it. This is the method used in [15], with a Green's function formulation giving the stress across the bed and is seen in the appendix.

Another extension of the model would be to tubes of varying radius. This would likely require the extension of the model to three dimensions. Further there is likely to be a choking effect if the radius decreases with height similar to that seen in steep-walled hoppers [45].

The final logical extension of the model is layered beds. The method of friction modeling used in this work does not work for layered beds as discontinuities arise at the boundary layers. Either the hoop stress would have to be altered to allow matching or a more sophisticated method could be used. One possible way to do this would be to solve the solid pressure equation separately in every layer and then match at the boundaries. 
The results of the model show the behavior found in experiments. Model extension would be required for further application of the model to natural or industrial such as volcanic eruptions or fluidized bed reactors. 


\section{Bibliography}

[1] M. P. McCormick, L. W. Thomason, and C. R. Trepte. Atmospheric effects of the Mt Pinatubo eruption. Nature, 373:399-404, 1995.

[2] R. B. Stothers. The great Tambora eruption in 1815 and its aftermath. Science, 224(4654):1191-1198, 1984.

[3] S. Carey, H. Sigurdsson, J. E. Gardner, and W. Criswell. Variations in column height and magma discharge during the may 18, 1980 eruption of Mount St. Helens. Journal of Volcanology and Geothermal Research, 43:99-112, 1990.

[4] T. Casadevall, W. Rose, T. Gerlach, L. P. Greenland, J. Ewert, R. Wunderman, and R. Symonds. Gas emissions and the eruptions of Mount St. Helens through 1982. Science, 221:1383-1385, 1983.

[5] P. W. Lipman and D. R. Mullineaux. The 1980 eruptions of Mount St. Helens, Washington. USGS, Washington D.C., 1982.

[6] C. S. Weaver, J. E. Zollweg, and S. D. Malone. Deep earthquakes beneath Mount St. Helens: Evidence for magmatic gas transport? Science, 221:13911394, 1983.

[7] F. Sigmundsson, S. Hreinsdóttir, A. Hooper, T. Árnadóttir, R. Pedersen, M. J. Roberts, N. Óskarsson, A. Auriac, J. Decriem, P. Einarsson, H. Geirsson, M. Hensch, B. G. Ófeigsson, E. Sturkell, H. Sveinbjörnsson, and K. L. Feigl. Intrusion triggering of the 2010 Eyjafjallajökull explosive eruption. Nature, 468(7322):426-430, 2010.

[8] A. R. Donovan and C. Oppenheimer. The 2010 Eyjafjallajökulll eruption and the reconstruction of geography. The Geographical Journal, 177(1):4-11, 2011. doi: 10.1111/j.1475-4959.2010.00379.x. 
[9] A. R. McBirney and T. Murase. Factors governing the formation of pyroclastic rocks. Bulletin of Volcanology, 34(2):372-384, 1970.

[10] J. Verhoogen. Mechanics of ash formation. American Journal of Science, 249: 729-739, 1951.

[11] A. R. McBirney. Conductivity variations and terrestrial heat-flow distribution. Journal of Geophysical Research, 68(23):6323-6329, 1963.

[12] R. S. J. Sparks. The dynamics of bubble formation and growth in magmas: A review and analysis. Journal of Volcanology and Geothermal Research, 3: $1-37,1978$.

[13] F. D. Bennett. On volcanic ash formation. American Journal of Science, 274: 648-661, 1974.

[14] G. A. Valentine. Stratified flow in pyroclastic surges. Bulletin of Volcanology, 49:616-630, 1987.

[15] A. C. Fowler, B. Scheu, W. T. Lee, and M. J. McGuinness. A theoretical model of the explosive framentation of vesicular magma. Proceedings of the Royal Society A, 466:731-752, 2010. doi: 10.1098.

[16] P. Papale. Strain-induced magma fragmentation in explosive eruptions. $\mathrm{Na-}$ ture, 397:425-428, 1999.

[17] J. F. Davidson, D. Harrison, and J. R. F. Guedes de Carvalho. On the liquidlike behaviour of fluidized beds. Annual Review of Fluid Mechanics, 9: 55-86, 1977.

[18] R. M. Davies and G. Taylor. The mechanics of large bubbles through extended liquids and through liquids in tubes. Proceedings of the Royal Society of London. Series A, Mathematical and Physical Sciences, 200(1062):375-390, 1950 .

[19] C. A. Willus. An experimental investigation of particle motion in liquid fluidized beds. PhD, California Institute of Technology, Pasadena, 1970.

[20] M. M. El-Kaissy and G. M. Homsy. Instability waves and the origin of bubbles in fluidized beds: Part 1: Experiments. International Journal of Multiphase Flow, 2(4):379-395, 1976. 
[21] A. F. Fortes, D. D. Joseph, and T. S. Lundgren. Nonlinear mechanics of fludization of beds of spherical particles. Journal of Fluid Mechanics, 177: 467-483, 1987.

[22] J. Happel and Pfeffer R. The motion of two spheres following each other in a viscous fluid. A.I.Ch.E. Journal, 6:129-133.

[23] G. Volpicelli, L. Massimilla, and F. A. Zenz. Nonhomogeneities in solid-liquid fluidization. Chemical engineering progress symposium series, 67:42-50, 1966.

[24] L. Zernow, N. Louie, H. Andersen, and P.J. Blatz. An experimental study of the reverse percolation lofting a sand medium. DNA Report 3210F, Whittaker Corporation, Sherman Oaks, 1973.

[25] F. A. Jr. Morrison. Transient non-darcy gas flow in a finite porous bed. Journal of Fluids Engineering, 99:779-781, 1977.

[26] R. H. Nilson. Transient fluid flow in porous media: Inertia-dominated to viscous-dominated transition. Journal of Fluids Engineering, 103(2):339-343, 1981.

[27] A. V. Anilkumar, R. S. J. Sparks, and B. Sturtevant. Geological implications and applications of high-velocity two-phase flow experiments. Journal of Volcanology and Geothermal Research, 56:145-160, 1993.

[28] A. V. Anilkumar. Experimental Studies of High-Speed Dense Dusty Gases. PhD, California Institute of Technology, Pasadena, CA, 1989.

[29] B. Cagnoli, A. Barmin, O. Melnik, and R. S. J. Sparks. Depressurization of fine powders in a shock tube and dynamics of fragmented magma in volcanic conduits. Earth and Planetary Science Letters, 204:101-113, 2002.

[30] D. Geldart. Types of gas fluidization. Powder Technology, 7:285-292, 1973.

[31] M. Alidibirov. A model for viscous magma fragmentation during volcanic blasts. Bulletin of Volcanology, 56:459-465, 1994.

[32] M. J. McGuinness, B. Scheu, and A. C. Fowler. Explosive fragmentation criteria and velocities for vesicular magma. Journal of Volcanology and Geothermal Research, 237-238:81-96, 2012. doi: 10.1016/j.jvolgeores.2012.05.019. 
[33] S. Dartevelle and G. A. Valentine. Transient multiphase processes during the explosive eruption of basalt through a geothermal borehole (namafjall, iceland, 1977) and implications for natural volcanic flows. Earth and Planetary Science Letters, 262:363-384, 2007. doi: 10.1016/j.epsl.2007.07.053.

[34] M. A. Biot. Mechanics of deformation and acoustic propagation in porous media. Journal of Applied Physics, 33:1482-1498, 1962. doi: 10.1063/1. 1728759.

[35] D. A. Nield and A. Bejan. Convection in porous media. Springer, New York, 3rd edition, 2006.

[36] D. Coelho, J.-F. Thovert, and P. M. Adler. Geometrical and transport properties of random packings of spheres and aspherical particles. Physical Review E, 55(2):1959-1978, 1997.

[37] G. J. Weir. The intrinsic cohesian of granular materials. Powder Technology, 104:26-36, 1999.

[38] Tomonari Kobayashi, Tetuichiro Mukai, Toshihiro Kawaguchi, Toshitugu Tanaka, and Yutaka Tsuji. DEM analysis on flow patterns of geldart's group a particles in fluidized bed. In Proceedings of World Congress on Particle Technology, volume 4, Sydney, Australia, July 2002.

[39] J. Crank. The Mathematics of Diffusion. Clarendon Press, Oxford, 2nd edition, 1975.

[40] M. Abramowitz and I. A. Stegun, editors. Handbook of Mathematical Functions. National Bureau of Standards, Washington D.C., 1964.

[41] M. J. McGuinness, C. P. Please, N. Fowkes, P. McGowan, L. Ryder, and D. Forte. Modelling the wetting and cooking of a single cereal grain. Research Report 98-28, Victoria University of Wellington, 1998.

[42] A. Friedman and K. Hllig. On the mesa problem. Journal of Mathematical Analysis and Applications, 123:564-571, 1987.

[43] O. Molerus. The role of science in particle technology. Powder Technology, 122:156-167, 2002. 
[44] T. Gröger, U. Tüzun, and D. M. Heyes. Modelling and measuring of cohesion in wet granular materials. Powder Technology, 133:203-215, 2003. doi: 10. 1016/S0032-5910(03)00093-7.

[45] G. J. Weir. A mathematical model for dilating, non-cohesive granular flows in steep-walled hoppers. Chemical Engineering Science, 59:149-161, 2004. doi: 10.1016/j.ces.2003.09.031.

[46] R. B. Malla and D. Schillinger. Experimental determination of friction coefficient and mobilization force in granular material packed in a cylinder. In Proceedings of the 2007 SEM Annual Conference \& Exposition on Experimental and Applied Mechanics, pages 1-9, Springfield, MA, 2007. 


\title{
Modelling the Initiation of Dust Eruptions
}

\author{
M.J. McGuinness, H. Singh \\ School of Mathematics, Statistics and Operations Research, Victoria University of Wellington, \\ New Zealand
}

\begin{abstract}
We present a new model for the initiation of high-speed eruptive two-phase dust flows in the laboratory. Shock-tube experiments have been conducted on beds of solid particles in nitrogen under high pressure, which are suddenly decompressed. Our model is successful in explaining the slab-like structures that are often observed during initiation of bed movement, by considering the interaction between the compressible flow of gas through the bed and the stress field in the particle bed, which ruptures when bed cohesion is overcome by the effective stress in the bed generated by the gas flow. Our model includes the effects of overburden and wall friction, and predicts that all layered configurations will rupture initially in this fashion, consistent with experimental observation. We also find that the modelled dependence of layer size on particle size is a good match to experiment.

Keywords: explosive fragmentation, mathematical model, dusty gas flow, shock tube, high speed two-phase flow
\end{abstract}

\section{Introduction}

Explosive volcanic activity is expressed in a wide range of forms, ranging from Hawaiian fire fountaining and Strombolian eruptions to highly energetic Vulcanian and Plinian eruptions. Fragmentation types may be roughly divided into two end-members depending on magma viscosity. In low-viscosity magma, proposed fragmentation mechanisms include bursting bubbles and foam instability (Verhoogen, 1951; Sparks, 1978; Mangan \& Cashman, 1996). On the other hand, in 
high-viscosity magma bubble growth is constrained by viscous forces resulting in over-pressurized vesicles. This magma tends to fragment in a brittle manner when the strength of the magma is exceeded, and this is usually taken to be due to the presence of pressurized vesicles (McBirney \& Murase, 1970; Heiken \& Wohletz, 1991; Gilbert \& Sparks, 1998; Cashman et al., 2000). These explosive eruptions are characterized by high-velocity flows of mixtures of solid particles and gas.

Laboratory experiments with shock-tube apparatus have been conducted in order to better understand fragmentation and flow processes in Vulcanian and Plinian eruptions. The materials used in these experiments vary from packed beds of spheres of glass and steel (Anilkumar et al, 1993; Anilkumar, 1989), through weakly cohesive artificial porous structures (Mader et al., 1994; Philips et al., 1995; Ichihara et al., 2002; Namiki \& Manga, 2005; Kameda et al., 2008), to competent natural samples of volcanic rock (Alidibirov, 1994; Martel et al., 2000; Spieler et al., 2004b; Scheu et al., 2006, 2008).

Shock-tube experiments conducted on samples of natural eruptive competent rock and weakly cohesive materials display a characteristic length-scale for primary fragmentation, so that slabs of solid material of approximately the same thickness successively break off from the remaining stationary material. When the sample is competent, it is necessary to anchor the initial sample into the shocktube with glue or by having a tight fit, to prevent it from immediately moving up the tube. Fragmenting sections, then, have simultaneously broken away from the remaining sample, and from the glued or tight-fitting sides.

A novel recent mathematical model of gas flow through porous rock has successfully explained the appearance of this hitherto perplexing lengthscale for competent rock samples (Fowler et al., 2009; McGuinness et al., 2012). In this modelling, a nonlinear diffusion equation was derived for the movement of gas through the rock sample, driven by the arrival at the sample surface of an expansion wave. The resulting variations in gas pressure stress the rock sample. This stress increases towards critical tensile strength while penetrating deeper into the still stationary sample. When tensile strength is exceeded, this occurs at a local maximum 
of stress some distance into the sample, causing a slab of material to fragment and move upwards. A key role is played in this previous modelling work by the mechanism that holds the remaining rock in place. Without glue or a tight fit, the model predicts that the maximum stress is at the base of the sample, not partway down, causing the entire sample to lift off. Indeed, this is what is observed in practice.

It may come as something of a surprise then, that when packed beds of small spheres with small cohesion are used instead of competent samples in shock-tube experiments, the very first mobilisation event observed is again the formation of horizontal cracks on a certain lengthscale at initiation of movement. The resulting slabs of beads are closely associated with the large-scale heterogeneities in flow density that are the main feature of the flow structure that subsequently develops (Anilkumar et al, 1993; Anilkumar, 1989; Cagnoli et al, 2002).

The tensile strengths of the bead beds considered is less than $100 \mathrm{~Pa}$, compared with a tensile strength of over $1 \mathrm{MPa}$ for rock. The over-pressures involved in the dust experiments are of the order of one bar, compared to about 100 bars for rocks.

The formation of slabs at initiation of movement of bead beds under transient gas pressure changes in shock tubes is not a feature of fluidised beds with gradually increased steady gas through-flow. As noted in Valverde et al (1998), the first fracture of such beds is always at the bottom of the bed. Smaller-scale slab structures in steady fluidised beds are only manifested for some classes of powders as slug flow features at gas flowrates significantly higher than required for fluidisation. Incompressible gas flow is a useful approximation in most modelling of fluidised beds, whereas compressible gas flow is central to the shock-tube setup since gas decompression is the cause of gas flow.

Our aim in this paper is to explain the formation of these slab structures at the onset of mobilisation of a low-cohesion bed of particles under the transient compressible gas flow imposed by the shock-tube setup, by using a modification of the modelling approach that has been so successful for shock-tube experiments on competent rock samples.

We review some details of the dust shock-tube experiments in the next section, 
then we introduce the mathematical model describing conservation of mass and momentum, and relating stress and strain, in the following section. In that section we derive a reduced set of equations describing gas pressures and stress, after rescaling and setting small parameters to zero. Solutions are presented in the following section, then the rupture condition is considered in the next section, followed by conclusions.

\section{Dust Experiments}

The experiments prompting this paper are reported by Anilkumar (1989) and Anilkumar et al (1993). A shock tube apparatus is used to fluidize packed beds of spheres sourced from a range of materials and with nitrogen gas as the working fluid, with a range of bed heights from $15-60 \mathrm{~cm}$, bed width of $3.8 \mathrm{~cm}$ with a square geometry, sphere diameters in the range $125-1000 \mu \mathrm{m}$, final speeds of $15-60 \mathrm{~m} / \mathrm{s}$, and accelerations in excess of $150 \mathrm{~g}$. In that apparatus, the timescale for pressure to drop by a factor of $e$ is about $1 \mathrm{~ms}$. In contrast, Cagnoli et al (2002) use smaller sphere diameters (38 and $95 \mu \mathrm{m}$ ) and smaller pressure drops (200-900 mbar), mobilised by sudden decompression of a dry air environment. In some of Anilkumar's experiments the bed of spheres rests on a solid base, in others it rests on a mesh with more high-pressure nitrogen below. The test section is pressurized to 2-3 bars gauge (barg, that is, bars above atmospheric pressure), and the pressure is released explosively at the top by rupturing a diaphragm separating the high pressure section from a $7-13 \mathrm{~m}$ long exhaust region at atmospheric pressure. Windows and cameras allow observation and recording of lofting packed beds of spheres as the gas expansion wave reaches the upper surface of the bed.

Figure 1 (taken from Anilkumar et al (1993)) shows initial mobilisation of the bed, with fractures dividing the bed into slabs being the first visible feature after bed expansion. Tellingly, Anilkumar (1989, p.27) notes that "initial bed expansion occurs along horizontal fractures that ...partition the bed into slabs". There is some variability of slab size, but typically each slab is ten to twenty particle diameters thick. The dynamics of the subsequent two-phase accelerating flow are complex, 
with particles falling from the bases of slabs and partitioning the fracture regions into bubbles, while particles at the tops of slabs are stable.

Slab thicknesses are observed to be approximately proportional to the square root of sphere diameter (Anilkumar, 1989), and this relationship is not hitherto understood. They are also observed to be independent of the initial over-pressure, considering two pressures, 2 and 3.1 barg. Slabs are the first-observed features of bed fragmentation in all experiments, and they are central to any major heterogeneity that may be seen later in the flow sequence. However, the reason they occur is hitherto unknown, and has been speculated to perhaps be due to an instability in the high-density two-phase flows that subsequently develop (Anilkumar, 1989; Dartevell and Valentine, 2007). However, the fractures giving slabs are the very first observed change in the bead beds, suggesting the cause may lie in the interaction of the compressible gas flow with the stationary bead bed.

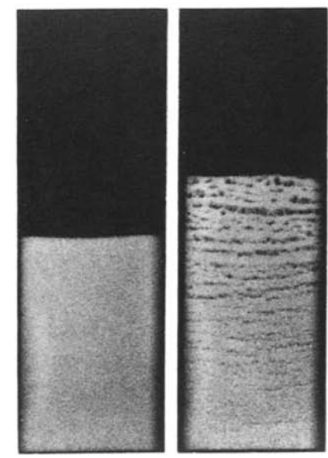

$2.8 \mathrm{~ms}$

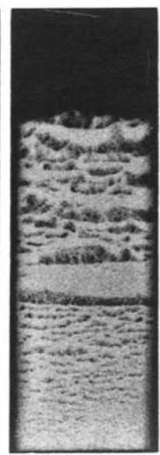

$4.0 \mathrm{~ms}$

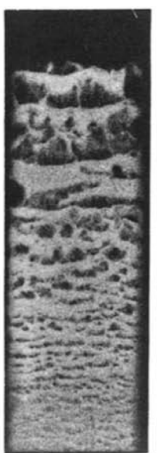

$5.2 \mathrm{~ms}$

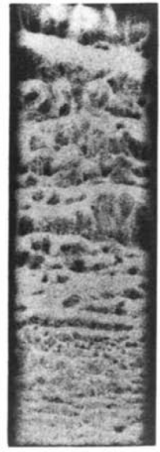

$6.5 \mathrm{~ms}$

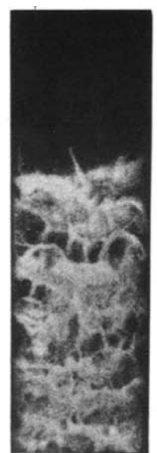

$7.8 \mathrm{~ms}$

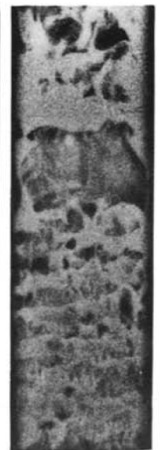

$11.5 \mathrm{~ms}$

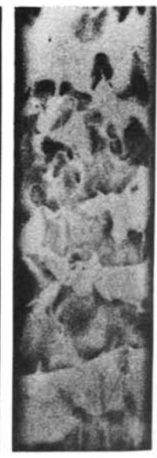

$14.0 \mathrm{~ms}$

Fig. 1. Initiation of lofting of a bed of glass beads of depth $60 \mathrm{~cm}$, initially at rest on a solid base, from Anilkumar et al (1993, Fig. 2). Initial overpressure is 2.1 bar, and bead diameter is $125 \mu \mathrm{m}$. The time since arrival of the expansion wave at the bed surface is shown below each snapshot, which is a view of the bed from the side. The camera view is shifted upwards once, at a time between 6.5 and $7.8 \mathrm{~ms}$. We are grateful to Dr. Anilkumar for permission to reproduce this figure.

When smaller (38 and $95 \mu \mathrm{m}$ diameter) spheres are used together with smaller pressure changes (Cagnoli et al, 2002), slabs are still seen when pressure differences are small, but are not as ubiquitous as in the work of Anilkumar (1989). 
Only bubbles are observed at very small pressure differences; at larger values there is a bubbly region in the upper part of the sample, then a slab region between the bubbly region and the undisturbed base.

Anilkumar et al (1993) and Anilkumar (1989) also experiment with various arrangements of two or three layers of beads, either of the same size but different density, or of the same density but different sizes. Cracks starting near the top of the bed are still the first visible change upon depressurization, in all configurations, but whether the cracks survive longer term or not depends on the bed configuration, and in the stable configurations Anilkumar refers to repacking of the layers that form initially, due to inertial or flow factors.

They find that if the lighter beads overly the heavier, the bed is unstable and slabs are ubiquitous and persistent in time, and the different layers separate first as primary slabs with larger void spaces between. The reverse bead arrangement with heavier ones on top leads initially to cracks forming, but then closing up so that eventually the entire bed lifts off as one plug, and remains very stable, with beads falling off from the bottom of the plug. If smaller beads overly larger beads, all of the same density, the bed is unstable and the layers of different sized beads separate from each other before themselves fragmenting into thinner slabs. An example of this from Anilkumar et al (1993) is reproduced in Fig. (2), and may be contrasted with the stable bead-size arrangement illustrated in Fig. (3) from the same paper. Well-mixed beds with three different bead sizes behave like a single-sized bead bed at the median bead size - slabs form and then separate.

Our aim in this paper is to explain the initial crack or slab formation in stationary beds of beads, seen in shock-tube experiments, and to explain the dependence of slab thickness on bead diameter. Subsequent flow development and repacking of certain configurations is beyond the scope of this paper.

\section{Mathematical Model of Erupting Dusts}

We consider the one-dimensional adiabatic upward compressible flow of gas through a deformable porous medium, the weakly cohesive stationary bed of 


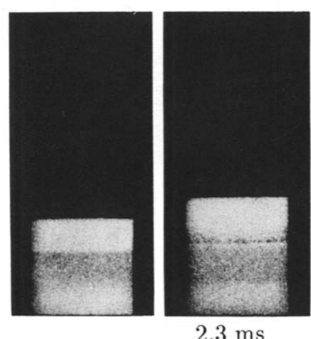

$2.3 \mathrm{~ms}$

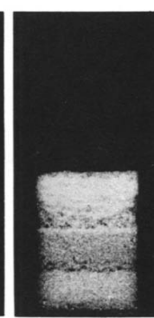

$3.3 \mathrm{~ms}$

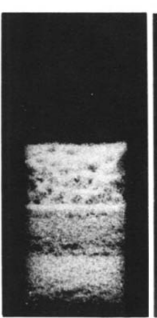

$4.3 \mathrm{~ms}$

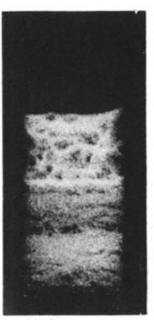

$5.0 \mathrm{~ms}$

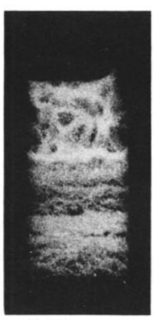

$5.7 \mathrm{~ms}$

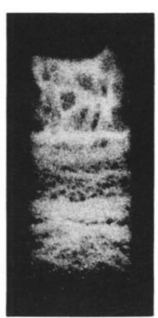

$6.2 \mathrm{~ms}$

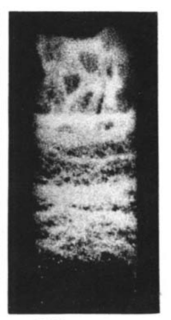

$6.7 \mathrm{~ms}$

Fig. 2. Initiation of lofting of a bed of glass beads of depth $3.8 \mathrm{~cm}$, initially at rest on a solid base, from Anilkumar et al (1993, Fig. 4b). Initial overpressure is 2.1 bar, and beads are unstably layered with smallest diameter $(250 \mu \mathrm{m})$ in the upper one-third of the bed, middle diameter $(500 \mu \mathrm{m})$ in the centre one-third of the bed, and the largest diameter $(750 \mu \mathrm{m})$ in the lower one-third. The time since arrival of the expansion wave at the bed surface is shown below each snapshot. We are grateful to Dr. Anilkumar for permission to reproduce this figure.
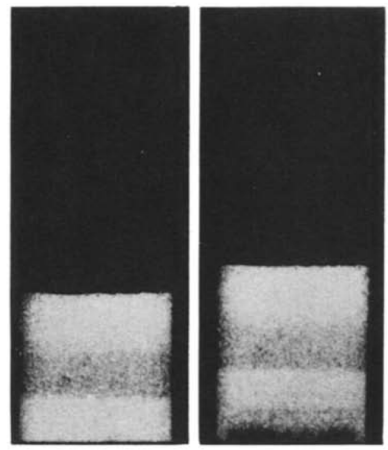

$2.9 \mathrm{~ms}$

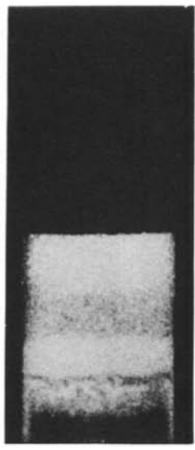

$4.1 \mathrm{~ms}$

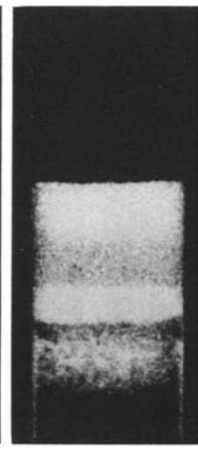

$5.4 \mathrm{~ms}$

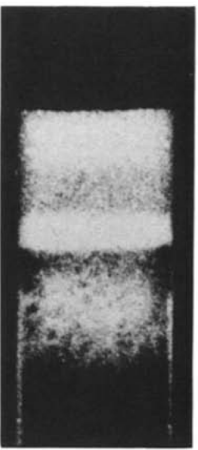

$6.7 \mathrm{~ms}$

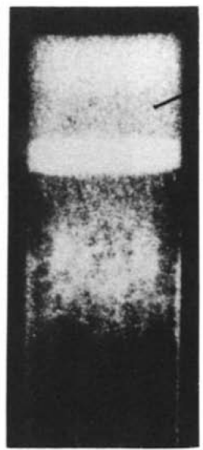

$8.0 \mathrm{~ms}$

Fig. 3. Initiation of lofting of a bed of glass beads of depth $3.8 \mathrm{~cm}$, initially at rest on a porous base (a screen) with high-pressure gas below, in and above the bed, from Anilkumar et al (1993, Fig. 4a). Initial overpressure is 2.1 bar, and beads are stably layered with smallest diameter $(250 \mu \mathrm{m})$ in the lower one-third of the bed, middle diameter $(500 \mu \mathrm{m})$ in the centre one-third of the bed, and the largest diameter $(750 \mu \mathrm{m})$ in the upper one-third. The time since arrival of the expansion wave at the bed surface is shown below each snapshot. Layers that form initially upon bed expansion are not readily visible in these reproductions, and are reported to repack and disappear during upwards movement. We are grateful to Dr. Anilkumar for permission to reproduce this figure. 
beads. This leads to a nonlinear diffusion equation for gas pressure or density, driven by a falling pressure at the upper surface of the bed, and a boundary value problem that determines the strain or stress in the weakly cohesive bead bed. We solve and find where the effective stress exceeds bed cohesion. The approach taken here is based on that used recently when modelling the fragmentation of competent rock samples when suddenly depressurized (Fowler et al., 2009; McGuinness et al., 2012), which in turn is based on seminal work by Biot (1956, 1962).

\subsection{Dimensional Model Equations}

Momentum conservation in the gas gives (Fowler et al., 2009)

$$
\rho \phi \mathrm{v}_{t}=-\phi p_{z}-A-D,
$$

where $z$ is the vertical coordinate (m) with origin at the base of the bed of beads, $p$ is gas pressure, $\rho$ is gas density, $\mathrm{v}$ is gas velocity, and $\mathrm{v}_{t}$ is gas acceleration. Typical values and ranges of values for material properties are listed in Table (1). The porosity is $\phi(z, t)$, and for a packed bed of stationary spheres in contact with each other it has the initial value $\phi_{0} \approx 0.4$, independent of sphere radius (Coelho et al, 1997). The term $A$ accounts for an added mass effect, which corresponds physically to the concept that moving a solid sphere through gas requires displacing the gas backwards (Biot, 1956). It can be written

$$
A=(1-\phi) C_{V M} \rho\left(\mathrm{v}_{t}-w_{t t}\right),
$$

where $C_{V M}$ is an order one constant relating the added mass density to the porosity and gas density. $w$ is the displacement of the solid beads, averaged over a representative elementary volume. The term $D$ has dimensions of pressure gradient and accounts for the interfacial drag when gas moves past solid particles, and when Darcy's law for flow in a porous medium is extended to Forcheimer's or Ergun's 
equation Ergun (1952) to include turbulent flow effects, takes the form

$$
D=\frac{\eta \phi^{2}}{k}\left(\mathrm{v}-w_{t}\right)+\frac{\rho C_{F} \phi^{3}}{\sqrt{k}}\left(\mathrm{v}-w_{t}\right)\left|\mathrm{v}-w_{t}\right|,
$$

where $\eta$ is the dynamic viscosity of the gas (about $18 \times 10^{-6} \mathrm{~Pa} . \mathrm{s}$ ), $k$ is the permeability of the bed (about $10^{-10} \mathrm{~m}^{2}$ ), and $C_{F}$ is the dimensionless Ergun coefficient (about 0.5).

Momentum conservation for the solid beads gives

$$
\rho_{s}(1-\phi) w_{t t}=(1-\phi) \sigma_{z}+A+D-\rho_{s}(1-\phi) g-F
$$

where $F$ accounts for friction at the walls of the container, and is taken to be of the form

$$
F=\frac{\mu_{f} w}{C_{c} D_{p}}
$$

with $\mu_{f}$ an effective shear modulus for the bed, and $C_{c}$ the length of the perimeter of the container cross-section. We set the effective shear modulus to the same value as the elastic modulus $E$, acting over a distance of one bead diameter, taking the walls to be in stick mode initially, before slip occurs. We have assumed the initial value of displacement $w$ is zero.

The second-last term on the right-hand side of eqn. (4) is called the overburden, and is negligible in Fowler et al. (2009) but is expected to play a central role at the lower over-pressures considered here for erupting dust experiments.

The term $\sigma$ is the vertical component of solid stress in the beads averaged over the cross-section, as detailed in Fowler et al. (2009), so that the stress-strain relationship is

$$
(1-\phi) \sigma=E w_{z}-\alpha p
$$

where $E$ is an elastic constant, and $\alpha$ is an order one elastic constant (Fowler et al., 2009).

The permeability of a bed of packed uniform spheres of diameter $D_{p}$ is the 
subject of the Karman-Cozeny relationship,

$$
k=\frac{D_{p}^{2} \phi^{3}}{72 \tau(1-\phi)^{2}},
$$

where $\tau$ is tortuosity. Coelho et al (1997) note that in a survey of a number of different experiments on packed beds, $k$ is observed to be in the range $(2-3) \times 10^{-3} D_{p}^{2} / 4$ $\mathrm{m}^{2}$ before bed expansion or fluidisation occurs. We here use

$$
k=0.625 \times 10^{-3} D_{p}^{2}
$$

for the permeability of the packed bed before any movement of beads occurs.

Conservation of gas mass gives

$$
(\rho \phi)_{t}+(\rho \phi \mathrm{v})_{z}=0
$$

and assuming adiabatic expansion of the gas, we can relate gas pressure $p(z, t)$ and density as

$$
\rho=\rho_{0}\left(\frac{p}{p_{0}}\right)^{\frac{1}{\gamma}},
$$

where $\gamma$ is the adiabatic index, with value 1.4 for nitrogen, and $\rho_{0}$ and $p_{0}$ are the initial values of gas density and pressure, before the diaphragm is ruptured in the shock tube.

Mass conservation for the beads can be expressed in the form

$$
\left(\rho_{s}(1-\phi)\right)_{t}+\left(\rho_{s}(1-\phi) w_{t}\right)_{z}=0
$$

but as in Fowler et al. (2009) this is considered to be satisfied in the following, by requiring constant $\phi=\phi_{0}$ and small displacements $w$, so is ignored in the remainder of this paper.

The initial conditions are $\mathrm{v}=0,-\sigma=p=p_{0}, w=w_{t}=0, \phi=0.4$ at $t=0$. The boundary conditions are $\mathrm{v}=w=0$ at the lower end of the bed $z=0$; and 
at the upper surface $z=l$ of the bed, pressure reduces with time as the expansion wave hits, modelled as

$$
-\sigma=p=p_{c}(t)=\left(p_{0}-p_{a}\right) \exp \left(-t / t_{c}\right)+p_{a},
$$

where $t_{c}$ is the timescale for pressure decay from the initial value $p_{0}$ to atmospheric

pressure $p_{a}$ at the bed surface, about $1 \mathrm{~ms}$ for the shock tube used by Anilkumar et al (1993).

\subsection{Rupture}

The gas pressure will drop at the surface of the bed from time zero when the expansion wave arrives there, and this pressure drop will penetrate the bed, so that for a time an increasing gas pressure difference will develop, between gas pressure in the bed and gas pressure at the surface. This pressure difference drives a change in stress in the solid, which increases until rupture of the bed occurs.

The bed is assumed to be held in place by

1. gravity (the overburden, or weight of the solid beads),

2. an intrinsic cohesion $S_{0}$, and

3. friction at the walls of the bed container.

Gravity and friction are already allowed for in the solid momentum conservation equation. Then, as in Fowler et al. (2009), the bed is taken to rupture when the effective stress $(1-\phi)(\sigma+p)$ exceeds the intrinsic cohesion $S_{0 c}$.

\subsection{Cohesion}

Granular materials have an intrinsic cohesion or tensile strength $\sigma_{0}$ due to Lifschitz-van der Waals forces, that varies with bead diameter (e.g., Xu \& Zhu (2006); Weir (1999); Jaraiz et al (1992); Tanneur et al (2008)). Glass beads in the size range we are considering are classified as Geldart Group A and B powders (Geldart, 1973; Jaraiz et al, 1992), which are readily fluidised, and where gravity effects are larger than Lifschitz-van der Waals interparticle forces, although for 
Group A powders these interparticle forces still play a part. The transition to Group B powders with larger beads, where interparticle forces are much smaller than gravity, occurs at about $100 \mu \mathrm{m}$ diameter for glass beads in the absence of factors like moisture altering cohesion.

There remain discrepancies between theoretical and measured values of cohesion for Group A and B powders. We present here a brief summary focussed on finding a reasonable range of possible values for the cohesion of the glass beads under consideration, with diameters of 125,500 and $750 \mu \mathrm{m}$.

Seminal work summarised by Molerus (2002) notes that the adhesion force at a contact for an unconsolidated or uncompressed glass powder bulk is (see also Molerus (1993))

$$
H_{0} \approx 9 \times 10^{-8} \mathrm{~N}
$$

and the number of contacts $N_{k}(\phi)$ is approximately six for each bead. The tensile strength of the bulk powder in the absence of any history of compression is then given by

$$
\sigma_{0}=\frac{(1-\phi) N_{k}(\phi)}{\pi D_{p}^{2}} H_{0} \approx \frac{10^{-7}}{D_{p}^{2}} \mathrm{~Pa} .
$$

Resulting values for the diameters we consider here are

$$
\begin{aligned}
\sigma_{0}=40 \mathrm{~Pa} \text { for } D_{p} & =50 \mu \mathrm{m}, \\
\sigma_{0} & =7 \mathrm{~Pa} \text { for } D_{p}=125 \mu \mathrm{m}, \\
\sigma_{0}=0.4 \mathrm{~Pa} \text { for } D_{p} & =500 \mu \mathrm{m}, \\
\sigma_{0} & =0.2 \mathrm{~Pa} \text { for } D_{p}=750 \mu \mathrm{m} .
\end{aligned}
$$

Note that at a diameter of about $200 \mu \mathrm{m}$ this becomes of the same order as the gravitational pressure $1 \mathrm{~Pa}$ due to one diameter of overburden.

For larger beads, there is evidence that the cohesion changes to become of the order of the gravitational pressure associated with one diameter of over-burden (Weir, 1999). This effective geometric cohesion is independent of the internal angle of friction, and is found (Weir, 1999) to give a match between the exact so- 
lution to the rigid-plastic flow equations and an extended Beverloo equation based on empirical observations of granular flow. It takes the value $\sigma^{0}=(1-\phi) \rho_{s} g D_{p} / 2$. It captures the purely geometric effect of a bead being in contact with its neighbours and the receptacle walls. The parameters $\sigma_{0}$ and $\sigma^{0}$ become of similar size when $D_{p} \approx 200 \mu$. We take the effective cohesion of an unconsolidated bed to be

$$
S_{0}=\sigma_{0}+\sigma^{0}
$$

\subsubsection{Consolidation}

Molerus (2002) notes that previous consolidation by a compressive force $N_{0}$ can change the contact surface area between beads due to plastic behaviour, and leads to the increased cohesive force

$$
H=H_{0}+\kappa N_{0},
$$

and the cohesion of a previously consolidated bed is then $S_{0 c}=S_{0}+\kappa \sigma_{N 0}=$ $\sigma_{0 c}+\sigma^{0}$, where

$$
\sigma_{0 c}=\frac{(1-\phi) N_{k}(\phi)}{\pi D_{p}^{2}} H=\sigma_{0}+\kappa \sigma_{N 0} \mathrm{~Pa}
$$

Experimental results (Molerus, 1993, 2002) suggest that cohesion is very sensitive to prior compression forces, and experimental values $\kappa \approx 0.3$ are also a good match to theoretical values, for $N_{0}$ values up to similar order to $H_{0}$ values.

Two possible sources of compression in the shock-tube experiments are the manner in which the bed is charged with high pressure gas $\sigma_{N C 0}$, and the gravity effect of overburden of material in the bed $\sigma_{N G 0}$, so that

$$
\sigma_{N 0}=\sigma_{N C 0}+\sigma_{N G 0}
$$

Charging with nitrogen during the setup of the experiment could lead to the bed being compressed, depending on the rate of charging. Anilkumar's test setup 
is charged from a high-pressure cylinder of nitrogen to a port above the top of the bed, controlled by a solenoid valve. A simple two-chamber pressure model allows us to estimate the compression effect on the bed, of charging the chamber. Consider the average pressure $p_{1}$ in the chamber above the bed, being charged at a constant rate $R_{1}(\mathrm{~Pa} / \mathrm{s})$, and bleeding at a rate $R_{2}\left(p_{1}-p_{2}\right)\left(\mathrm{s}^{-1}\right)$ into the second chamber which is the bed itself with an average bed pressure $p_{2}$. Conservation of gas mass gives the equations

$$
\begin{aligned}
& \frac{d p_{1}}{d t}=R_{1}-R_{2}\left(p_{1}-p_{2}\right), \\
& \frac{d p_{2}}{d t}=R_{2}\left(p_{1}-p_{2}\right) .
\end{aligned}
$$

Subtracting the second equation from the first, and taking both pressures to start from atmospheric pressure, gives the following differential equation for $\Delta p=$ $p_{1}-p_{2}$

$$
\frac{d}{d t} \Delta p=R_{1}-2 R_{2} \Delta p
$$

with initial condition $\Delta p=0$, and the solution is

$$
\Delta p=\frac{R_{1}}{2 R_{2}}\left(1-e^{-2 R_{2} t}\right),
$$

which starts at zero and rises towards the asymptotic value $R_{1} /\left(2 R_{2}\right)$.

This asymptotic value for $\Delta p$ is used as a proxy for the compression imposed on the bed by charging with nitrogen. We now estimate values for $R_{1}$ and $R_{2}$ to provide a value for this compression.

Discharge from a typical bed setup takes about $40 \mathrm{~ms}$ in Anilkumar's experiments, so taking a timescale of $40 \mathrm{~ms}$ to charge the bed with a given pressure difference imposed suddenly above the bed, gives $R_{2} \approx 25 \mathrm{~s}^{-1}$.

If the charge valve is opened gradually over a time of two minutes for a total charge of $200 \mathrm{kPa}$, the charging rate is $R_{1}=1660 \mathrm{~Pa} / \mathrm{s}$.

The compressive pressure on the bed associated with charging is then estimated at $\sigma_{N C 0}=R_{1} /\left(2 R_{2}\right) \approx 33 \mathrm{~Pa}$. For beads bigger than $50 \mu \mathrm{m}$ the cohesion $\sigma_{0 c}$ 
is then significantly altered from $\sigma_{0}$. If the charge valve is opened over a period of two seconds rather than two minutes, the same calculation leads to the value $\sigma_{N C 0} \approx 2000 \mathrm{~Pa}$. There is clearly a large degree of variability in this value, depending on experimental conditions and setup, with our calculations suggesting that a reasonable range of values for $\sigma_{N C 0}$ is 30-2000 Pa.

The second possible source of compression is the overburden in the bed. The pressure due to solid overburden at dimensionless height $z$, is

$$
\sigma_{N G 0}=(1-\phi) \rho_{s} g l(1-z) .
$$

This ranges in value from zero to about $670 \mathrm{~Pa}$, from top to bottom of the bed.

Note that here we are not modelling the effect of overburden on momentum conservation or a force balance, as this is already done above. We are considering the effect of the compression associated with overburden on the contact area between beads, and hence on bed cohesion. A similar calculation is made by Orband \& Geldart (1997) to explain cohesions observed in measurements made on $64 \mu \mathrm{m}$ glass ballotini that are six times larger than the unconsolidated values.

Then the compression term is

$$
\kappa \sigma_{N 0}=\kappa \sigma_{N C 0}+\kappa(1-\phi) \rho_{s} g l(1-z) \approx(9-600)+670(1-z) \mathrm{Pa} .
$$

The effects of prior compaction by vibration of the bed can be significant for smaller sized beads, according to a recent study by Xu \& Zhu (2006), where tensile strength measured by the overshoot pressure at incipient bed fluidisation varies by factors of up to four as prior compaction varies.

A Warren Spring-Bradford apparatus is used by Orband \& Geldart (1997) to measure the cohesion of freely-flowing powder samples with mean sizes from 20 to $120 \mu \mathrm{m}$. They find a range of values, all greater than $100 \mathrm{~Pa}$, with almost constant apparent cohesion above a critical size. For glass ballotini at $67 \mu \mathrm{m}$ diameter they measure a tensile strength of $140 \mathrm{~Pa}$.

A number of other factors can affect the apparent cohesion of a powder, includ- 
ing the amount of moisture present, vibration (Xu \& Zhu, 2006), and electrostatic forces. Emery et al (2009) discuss the various possible effects of moisture on tensile strength, ranging from liquid bridging across particle contacts to decreased electrostatic forces. Mikami et al (1998) develop numerical simulations of the effects of moisture on tensile strength in a fluidised bed, and also discuss the modelling of wall friction. Weber (2004) explores the importance of liquid bridging forces, in a study of the pressure overshoot and hysteresis often seen at incipient fluidisation in plots of pressure difference across a bed versus steady fluid velocity. He finds that particle-particle cohesion dominates wall friction and cohesion with the bottom of the bed container, although the latter do have some effect on the overshoot.

However, it is unclear what steps were taken to dry the beads used in Anilkumar's experiments, and what the charging method was.

To summarise, the criterion for bed rupture is given in terms of the effective cohesion of a possibly consolidated bed as

$$
(1-\phi)(\sigma+p)>S_{0 c}
$$

where

$$
S_{0 c}=\sigma_{0 c}+\sigma^{0}=\sigma_{0}+\kappa \sigma_{N 0}+\sigma^{0}
$$

where $\sigma_{0}$ is given by eqn (18), $\kappa \sigma_{N 0}$ is given by eqn (19), and $\sigma^{0}=(1-\phi) \rho_{s} g D_{p} / 2$. 


\subsection{Nondimensional Model Equations}

The dimensional model equations are rescaled and nondimensionalized to variables with a tilde on top, by the transformations

$$
\begin{aligned}
\rho=\rho_{0}(1-\lambda \tilde{\rho}), & p_{c}=p_{0}\left(1-\gamma \lambda \tilde{p}_{c}\right) \\
p=p_{0}(1-\gamma \lambda \tilde{p}), & p_{a}=p_{0}\left(1-\gamma \lambda \tilde{p}_{a}\right) \\
z=l \tilde{z}, & \sigma=p_{0}(1-\gamma \lambda \tilde{\sigma}) \\
t=t_{0} \tilde{t}, & t_{0}=\frac{\lambda l}{\mathrm{v}_{0}} \\
\mathrm{v}=\mathrm{v}_{0} \tilde{\mathrm{v}}, & \mathrm{v}_{0}=\frac{k_{0} \rho_{s}\left(1-\phi_{0}\right) g}{\eta \phi_{0}} \\
k=k_{0} \tilde{k}, & k_{0} \approx 1.6 \times 10^{-10} \mathrm{~m}^{2} \\
w=w_{0} \tilde{w}, & w_{0}=\frac{p_{0} \gamma \lambda l}{E} \\
A=A_{0} \tilde{A}, & A_{0}=\frac{\rho_{0} v_{0}^{2}}{\lambda l} \\
D=D_{0} \tilde{D}, & D_{0}=\frac{p_{0} \gamma \lambda}{l} \\
\lambda=\frac{\left(1-\phi_{0}\right) \rho_{s} g l}{\gamma p_{0}} &
\end{aligned}
$$

Pressure changes have been scaled on overburden pressure relative to the initial gas pressure, through the parameter $\lambda$, and velocity scale is chosen to simplify the drag term that dominates the interaction between gas and solid phases.

The resulting dimensionless equations are

$$
\begin{aligned}
v_{1} \phi(1-\lambda \tilde{\rho}) \tilde{\mathrm{v}}_{\tilde{t}} & =\phi \tilde{p}_{\tilde{z}}-v_{1} \tilde{A}-\tilde{D} \\
{[(1-\lambda \tilde{\rho}) \phi]_{\tilde{t}} } & =-\frac{\partial}{\partial \tilde{z}}[\lambda(1-\lambda \tilde{\rho}) \phi \tilde{\mathrm{v}}] \\
\varepsilon(1-\phi) \tilde{w}_{\tilde{t}} & =-(1-\phi) \tilde{\sigma}_{\tilde{z}}+v_{1} \tilde{A}+\tilde{D}-G-\lambda_{f} \tilde{w} \\
(1-\phi)(1-\gamma \lambda \tilde{\sigma}) & =\gamma \lambda \tilde{w}_{\tilde{z}}-\alpha(1-\gamma \lambda \tilde{p}), \\
\tilde{A} & =(1-\phi) C_{V M}(1-\lambda \tilde{\rho})\left(\tilde{\mathrm{v}}_{\tilde{t}}-\delta \tilde{w}_{\tilde{t}}\right) \\
\tilde{D} & =\frac{\phi^{2}}{\phi_{0}}\left(\frac{\tilde{\mathrm{v}}-\delta \tilde{w}_{\tilde{t}}}{\tilde{k}}\right)
\end{aligned}
$$




$$
\begin{aligned}
& +\operatorname{Re}_{p} c_{F} \frac{\phi^{3}}{\phi_{0}}(1-\lambda \tilde{\rho})\left(\frac{\left(\tilde{\mathrm{v}}-\delta \tilde{w}_{\tilde{t}}\right)\left|\tilde{\mathrm{v}}-\delta \tilde{w}_{\tilde{t}}\right|}{\sqrt{\tilde{k}}}\right) \\
G= & \frac{1-\phi}{1-\phi_{0}}
\end{aligned}
$$

with the first three equations representing conservation of gas momentum, gas mass, and solid momentum respectively. Dimensionless pressure at the surface of the bed satisfies

$$
\tilde{p}_{c}=\tilde{p}_{a}(1-\exp (-a \tilde{t}))
$$

and parameters and their typical values are listed in Table (2).

\begin{tabular}{|c|l|l||l|}
\hline Symbol & Meaning & range & Typical value \\
\hline$C_{c}$ & perimeter of container & & $0.15 \mathrm{~m}$ \\
$c_{F}$ & Ergun coefficient & & 0.5 \\
$C_{V M}$ & added mass const & & 1 \\
$D_{p}$ & bead diameter & $30-1000 \mu \mathrm{m}$ & $500 \mu \mathrm{m}$ \\
$E$ & elastic constant & & $10^{11} \mathrm{~Pa}$ \\
$k_{0}$ & permeability scale & $4-40 \times 10^{-11} \mathrm{~m}^{2}$ & $1.6 \times 10^{-10} \mathrm{~m}^{2}$ \\
$l$ & bed depth & $0.15-0.64 \mathrm{~m}$ & $0.15 \mathrm{~m}$ \\
$p_{0}$ & initial gas pressure & $2-3$ bara & $3 \mathrm{bara}$ \\
$t_{c}$ & chamber relaxation time & & $1 \mathrm{~ms}$ \\
$\eta$ & gas viscosity & & $1.8 \times 10^{-5} \mathrm{~Pa} \mathrm{~s}$ \\
$\gamma$ & specific heat ratio & & 1.4 \\
$\rho_{0}$ & initial gas density & $1-2.3 \mathrm{~kg} \cdot \mathrm{m}^{-3}$ & $2.3 \mathrm{~kg} \cdot \mathrm{m}^{-3}$ \\
$\rho_{s}$ & solid density (glass) & & $2.5 \times 10^{3} \mathrm{~kg} \mathrm{~m}^{-3}$ \\
$\mu_{f}$ & friction shear modulus & & $10^{11} \mathrm{~Pa}$ \\
$\phi_{0}$ & initial porosity & & 0.4 \\
\hline
\end{tabular}

Table 1. Typical values of the physical constants of the model. The gas properties are those of nitrogen at room temperature.

The adiabatic law becomes

$$
1-\lambda \tilde{\rho}=(1-\lambda \gamma \tilde{p})^{1 / \gamma}
$$




\begin{tabular}{|c|l||l|}
\hline Parameter & Formula & Typical value \\
\hline$a$ & $\frac{t_{0}}{t_{c}}$ & 2.5 \\
$\operatorname{Re}_{p}$ & $\frac{\rho_{0} \mathrm{v}_{0} \sqrt{k_{0}}}{\eta}$ & 0.5 \\
$t_{0}$ & $\frac{\lambda l}{\mathrm{v}_{0}}$ & $2.5 \mathrm{~ms}$ \\
$\mathrm{v}_{0}$ & $\frac{k_{0} p_{0} \gamma \lambda}{\eta \phi_{0} l}$ & $0.3 \mathrm{~m} / \mathrm{s}$ \\
$\beta$ & $\alpha+\phi_{0}$ & 1 \\
$\delta$ & $\frac{p_{0} \gamma}{E}$ & $4 \times 10^{-6}$ \\
$\varepsilon$ & $\frac{\rho_{s} \mathrm{v}_{0}^{2}}{E \lambda^{2}}$ & $9 \times 10^{-5}$ \\
$\lambda$ & $\frac{\left(1-\phi_{0}\right) \rho_{s} g l}{\gamma p_{0}}$ & 0.005 \\
$\lambda_{f}$ & $\frac{\mu_{f} l^{2}}{C_{c} D_{p} E}$ & 308 \\
$v_{1}$ & $\frac{\rho_{0} \mathrm{v}_{0}^{2}}{p_{0} \gamma \lambda^{2}}$ & 0.02 \\
\hline
\end{tabular}

Table 2. Definitions and typical values of the parameters of the model.

The bed rupture condition (20) becomes

$$
(1-\phi)\left[p_{0}(1-\gamma \lambda \tilde{\sigma})+p_{0}(1-\gamma \lambda \tilde{p})\right]>S_{0 c},
$$

and using eqn (26) this becomes

$$
p_{0} \gamma \lambda \tilde{w}_{\tilde{z}}+(1-\beta) p_{0}(1-\gamma \lambda \tilde{p})>S_{0 c},
$$

where $\beta \equiv \phi+\alpha=O(1)$ so that approximately, rupture occurs when

$$
\tilde{w}_{z}>\tilde{S}_{0 c} \equiv \frac{S_{0 c}}{p_{0} \gamma \lambda}
$$

We now drop the tilde notation, so that unless otherwise stated, variables are dimensionless from now on. 


\subsection{Reduced Equations for Rupture}

Since $\lambda$ is small, the adiabatic law (30) may be approximated as

$$
p=\rho
$$

Considering the initiation of movement of beads, we set $\phi=\phi_{0}$ constant everywhere. Then neglecting terms containing the small parameters $v_{1}, \delta$, and $\epsilon$, we obtain the reduced set of dimensionless equations

$$
\begin{aligned}
\phi_{0} p_{z} & =D \\
p_{t} & =\mathrm{v}_{z} \\
\left(1-\phi_{0}\right) \sigma_{z} & =D-1-\lambda_{f} w \\
\left(1-\phi_{0}\right)(1-\gamma \lambda \sigma) & =\gamma \lambda w_{z}-\alpha(1-\gamma \lambda p), \\
D & =\phi_{0} \frac{\mathrm{v}}{k}+\operatorname{Re}_{p} c_{F} \phi_{0}^{2} \frac{\mathrm{v}^{2}}{\sqrt{k}} .
\end{aligned}
$$

The last equation may be further simplified by noting that $\operatorname{Re}_{p} c_{F} \phi_{0} \approx 0.1$ is relatively small, so that $D \approx \phi_{0} \mathrm{v} / k$. This in combination with eqns (34) and (35) gives the linear diffusion equation for nondimensional gas flow,

$$
p_{t}=\left(k p_{z}\right)_{z}
$$

with boundary conditions $p_{z}=0$ at $z=0$, and $p=p_{c}(t)=p_{a}(1-\exp (-a t))$ at $z=1$, and initial condition $p=0$. A typical nondimensional value for $p_{a}$ is 90 .

The steady-state solid momentum equation (36) combined with a differentiated stress-strain equation (37) gives the following nondimensional boundaryvalue problem for solid displacement $w$,

$$
w_{z z}-\lambda_{f} w=-\beta p_{z}+1
$$

with boundary conditions $w=0$ at $z=0$, and $w_{z}=0$ at $z=1$. We will use $\beta=1$. Strain in the solid is driven by gas pressure changes through the term $p_{z}$, and by 
overburden through the term 1.

The reduced problem has separated into two problems, the first being a soluble linear pressure diffusion equation. The second, boundary-value problem, may be solved to find $w$ once pressure is known from the solution to the diffusion problem. Then the rupture condition (33) can be checked at each value of time, to find out when and where the first bed rupture occurs.

\section{Solutions}

We now consider analytic and numerical solutions, firstly to the gas diffusion problem in equation (39), and secondly to the boundary-value problem (40).

\subsection{Diffusion Equation Solutions}

We solve the linear diffusion problem (39) with a constant scaled permeability $k=1$, corresponding to a bed of beads of uniform diameter. Taking a Laplace transform in time gives

$$
P(z, s)=\int_{0}^{\infty} p(z, t) e^{-s t} d s
$$

reduces the problem to the ordinary differential equation

$$
P_{z z}=s P
$$

with boundary conditions $P_{z}=0$ at $z=0, P=\frac{a p_{a}}{s(s+a)}$ at $z=1$. The solution in transform space is

$$
P=\frac{a p_{a}}{s(s+a)} \frac{\cosh (\sqrt{s} z)}{\cosh (\sqrt{s})} .
$$

Inverting this is possible by an extension of work presented in Crank (1975, eqn 2.53), and gives an infinite sum of erfc functions, which converges rapidly for all except large values of $t$. A small-time expansion follows from a consideration of 
the large- $s$ expansion of $P$, as

$$
P \sim \operatorname{ap}_{a}\left(\frac{\exp (-\sqrt{s}(1-z))}{s^{2}}\right), \quad s \rightarrow \infty
$$

with inverse transform (Abramowitz \& Stegun, 1972, 29.3.86)

$$
p=4 a p_{a} t \dot{\mathrm{i}}^{2} \operatorname{erfc}\left(\frac{1-z}{2 \sqrt{t}}\right)
$$

where $\mathrm{i}^{2}$ erfc is an integrated error function:

$$
\mathrm{i}^{2} \operatorname{erfc}(x)=\frac{1}{\sqrt{\pi}} \int_{x}^{\infty}(t-x)^{2} \mathrm{e}^{-t^{2}} d t
$$

This approximation to the solution, expected to be valid for early to moderate times, can also be written in the form

$$
p=a p_{a}\left[\left(t+\frac{(1-z)^{2}}{2}\right) \operatorname{erfc}\left(\frac{1-z}{2 \sqrt{t}}\right)-(1-z) \sqrt{\frac{t}{\pi}} e^{-\frac{(1-z)^{2}}{4 t}}\right]
$$

where

$$
\operatorname{erfc}(x) \equiv \frac{2}{\sqrt{\pi}} \int_{x}^{\infty} e^{-t^{2}} d t
$$

Numerical solutions, comparing the above asymptotic approximation with full numerical solutions of the linear diffusion equation, confirm that this is an excellent approximation for early times, as illustrated in Fig. (4), where the pressure difference $\Delta p=p_{c}-p$ is plotted against $z$, for five dimensionless times stepping evenly from zero to $2 \times 10^{-6}$ and a bead diameter of $500 \mu \mathrm{m}$.

\subsection{Boundary-Value Solutions}

Following McGuinness et al. (2012), we solve the boundary-value problem (40) for solid strain $w_{z}$ with $\beta=1$, given the early-time pressure solution (43). 


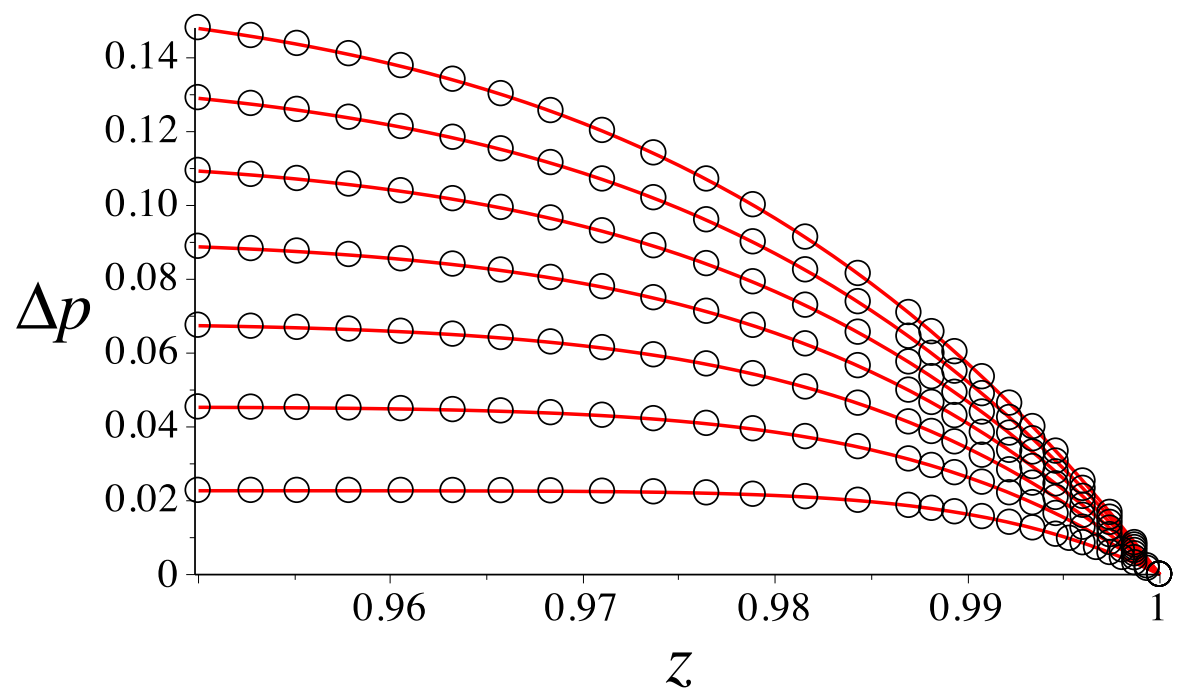

Fig. 4. Numerical solutions $p$ to the gas diffusion equation (39) with $k=1$, obtained using the pdsolve command in Maple, and converted to the form $\Delta p=$ $p_{c}-p$, plotted against nondimensional bed height $z$, for nondimensional times evenly spaced from zero to $7 \times 10^{-4}$. Since $t_{0}=0.0025 \mathrm{~s}$, the dimensional time is up to $1.8 \times 10^{-6} \mathrm{~s}$. The plot shows the excellent match between numerical solutions (solid line) and the analytical early-time approximation given by equation (43) (circles). 
The Green's function $G\left(z, z_{0}\right)$ for solving

$$
w_{z z}-\lambda_{f} w=1-p_{z}
$$

with $p_{z}$ prescribed as a function of $z$ at a given time, and boundary conditions $w(0)=0, w_{z}(1)=0$, satisfies

$$
G_{z z}-\lambda_{f} G=\delta_{D}\left(z-z_{0}\right)
$$

where $\delta_{D}$ is the Dirac delta function, with the usual continuity conditions across the jump at $z=z_{0}$. $G$ is given by the formula

$$
G\left(z, z_{0}\right)=-\frac{1}{v D} \begin{cases}\sinh (v z) \cosh \left(v\left(1-z_{0}\right)\right), & z \leq z_{0} \\ \sinh \left(v z_{0}\right) \cosh (v(1-z)), & z>z_{0}\end{cases}
$$

where $D=\cosh v$ and $v=\sqrt{\lambda_{f}}$.

Then $w_{z}$ is obtained by the quadrature

$$
w_{z}(z)=\int_{0}^{1} G_{z}\left(z, z_{0}\right)\left[1-p_{z}\left(z_{0}\right)\right] d z_{0},
$$

where the derivative of the Green's function is

$$
G_{z}\left(z, z_{0}\right)=-\frac{1}{D} \begin{cases}\cosh (v z) \cosh \left(v\left(1-z_{0}\right)\right), & z \leq z_{0} \\ -\sinh \left(v z_{0}\right) \sinh (v(1-z)), & z>z_{0}\end{cases}
$$

This formula has been tested in Matlab by comparing with a direct numerical solution to (40) using the routine bvp4c.

Numerical solutions for $w_{z}$ computed using the quadrature (46) and the pressure solution (42) with typical parameter values, are plotted in Fig. (5). The value $\lambda_{f}=308$ is large enough that the outer solution $w \sim-\left(1-p_{z}\right) / \lambda_{f} \ll 1$ obtained by dividing through by $\lambda_{f}$ and neglecting $w_{z z}$ obtains over much of the $z$ range, giving a small positive value for $w_{z}$. 


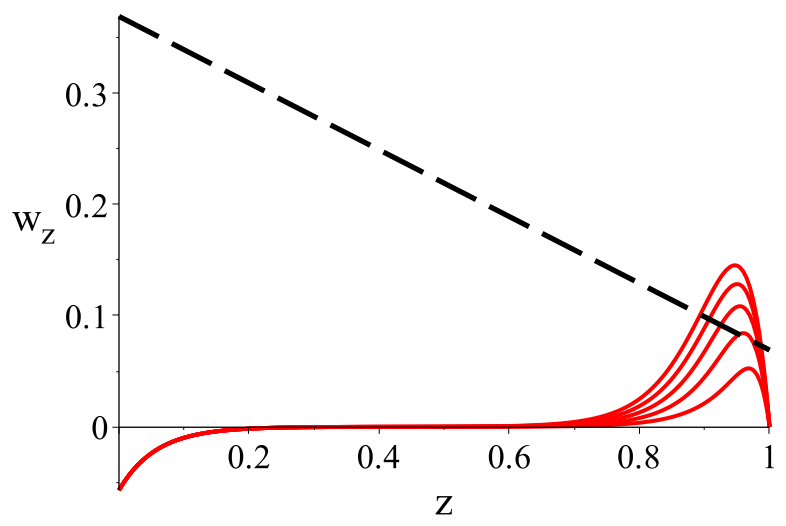

Fig. 5. Strain $w_{z}$ versus $z$ (solid lines), at evenly spaced dimensionless times from zero to $t=25 \times 10^{-4}$, compared with the rescaled tensile strength (dashed line). Parameter values are as listed in Tables (1) and (2). Strain increases with time. Bed rupture is observed at about $t=10 \times 10^{-4}$, when $w_{z}$ first exceeds the cohesion.

\subsection{The Shape of Strain Solutions}

To allow the model to be applied to a range of situations, with varying bed permeability and/or bead density, the shape of solutions $w_{z}$ will be explored using singular perturbation theory. The large size of $\lambda_{f}$ will be leveraged; small values are perturbations of the zero friction case discussed in the next subsection.

The boundary-value problem is considered in the form

$$
\epsilon^{2} w_{z z}-w=\epsilon^{2}\left(1-p_{z}\right)
$$

where $\epsilon^{2}=1 / \lambda_{f} \ll 1$ is small.

The outer solution is a good approximation to $w$ whenever the second derivative term can be ignored, and is small:

$$
w^{\text {outer }}=\epsilon^{2}\left(p_{z}-1\right) \approx 0
$$

giving the positive but small outer solution for strain

$$
w_{z}^{\text {outer }}=\epsilon^{2} p_{z z} \approx 0
$$


Inner solutions occur near $z=0$ and near $z=1$. Near $z=0$, we rescale $z=\epsilon z^{*}$ so that $z$ is close to zero and $p_{z}$ is approximated by $p_{z}(0)$ which is zero,

$$
w_{z^{*} z^{*}}^{\mathrm{inner} 1}-w^{\mathrm{inner} 1} \approx \epsilon^{2}\left(1-p_{z}(0)\right)=\epsilon^{2} .
$$

Solving this with $w(0)=0$ and requiring it to match the outer solution gives

$$
w^{\text {inner1 }}=\epsilon^{2}\left(e^{-z / \epsilon}-1\right),
$$

giving the strain near $z=0$ as

$$
w_{z}^{\mathrm{inner} 1}=-\epsilon e^{-z / \epsilon},
$$

which explains the small uptick seen in $w_{z}$ near origin in Fig (5).

Near $z=1$, we rescale $\epsilon z^{*}=1-z$, and we acknowledge the importance of the $p_{z}$ term by taking it to be large, as $p_{z}=\frac{\tilde{p}_{z}}{\epsilon^{2}}$, giving

$$
w_{z^{*} z^{*}}^{\mathrm{inne} 2}-w^{\mathrm{inner} 2} \approx \epsilon^{2}-\tilde{p}_{z}(z) \approx-\tilde{p}_{z}(z) .
$$

This is the same as the boundary value problem that arises in Fowler et al. (2009) when rupturing competent rock (noting that there is a sign difference between the scaled pressures used). There a powerful iterative general argument is given for the shape of $w_{z}^{\text {inner2 }}$ having a unique maximum, as seen near $z=1$ in Fig (5).

These arguments that $w_{z}$ has the general shape seen in Fig (5) apply for general shapes of $p(z)$ that are monotonic increasing, so that the unique local maximum in $w_{z}$ that rises to meet a threshold cohesion is common to a range of modelling situations, in particular if permeability $k$ is allowed to vary with depth, since the shape of $p(z)$ would be similar. 


\subsection{No Wall Friction}

Solutions to the boundary-value problem (40) are particularly straightforward if wall friction is ignored, that is, $\lambda_{f}=0$. Then

$$
w_{z z}=1-p_{z}
$$

which can be integrated from $z$ to 1 , to get

$$
w_{z}=p_{c}-p+z-1,
$$

with rupture condition

$$
w_{z}>S_{0 c},
$$

so that rupture occurs when

$$
p_{c}-p>1-z+S_{0 c}
$$

That is, in the absence of wall friction, there is a nice physical interpretation of the rupture condition, that bed rupture is predicted to occur when the difference between the gas pressure at depth $z$ and the gas pressure $p_{c}$ at the surface of the bed matches the overburden $1-z$ (in nondimensional form) at that depth, plus the effective cohesion, $S_{0 c}$.

This rupture is illustrated for the choice $\sigma_{N C 0}=500$ in Fig. (6), where it can be seen that $w_{z}$ has a unique local maximum due to the combination of a monotonically decreasing $p_{c}-p$, and a monotonically increasing $z-1$, as $z$ increases.

This behaviour for strain differs from that for the rupture of competent rocks found in Fowler et al. (2009) and McGuinness et al. (2012), where the effect of glue or a tight fit at the walls of the shock tube was crucial to obtaining a local maximum in $w_{z}$, and hence obtaining fragmentation at some finite depth rather than at the bottom of the sample. In contrast, $w_{z}$ has a local maximum now with zero wall friction. The difference here is that gravity or overburden is important, giving the crucial $z-1$ term, whereas gravity was correctly neglected in Fowler 
et al. (2009) due to the relatively larger over pressures required to overcome the tensile strength of competent rock.

In regions away from $z=1$, where the pressure has not had time to change yet, $w_{z} \approx z-1+p_{c}$ in this zero wall friction case, straight lines of slope one, moving upwards as $p_{c}$ increases with time, as illustrated in Fig. (7) by the bottom-most line, and as suggested by the smaller $z$ values in Fig. (6).

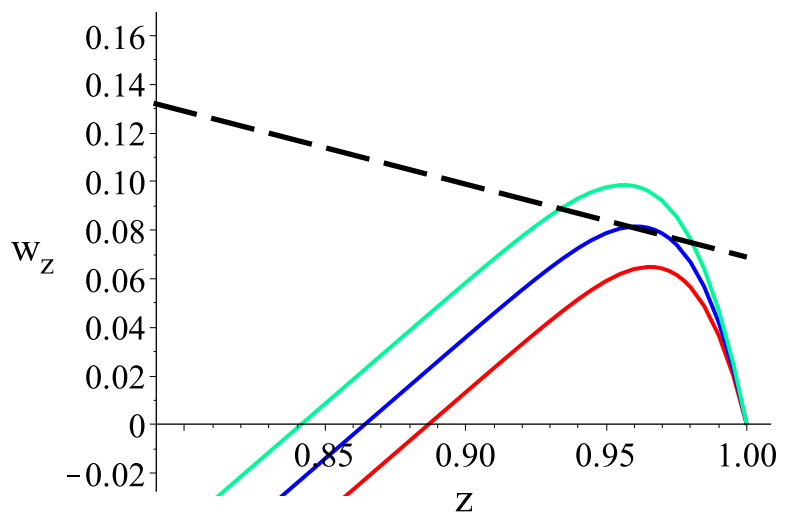

Fig. 6. Strain $w_{z}$ versus $z$ (solid lines), at dimensionless times $t=3,4,5 \times 10^{-4}$, compared with the tensile strength (dashed line). Wall friction is set to zero, and other parameter values are as listed in the tables. Strain increases with time. Bed rupture is observed at about $t=4 \times 10^{-4}$, and $z \approx 0.974$.

The effect of varying the friction term between zero and 308 is explored in Fig. (7), where $w_{z}$ is graphed versus $z$, for one value of time and several values of $\lambda_{f}$. It can be seen that while the effect of varying friction is noticeable away from $z=1$, it is relatively small near $z=1$. There is a delay in rupture times as $\lambda_{f}$ increases, but the location of the rupture is not very sensitive to $\lambda_{f}$. This is emphasised in Fig. (8), where the time of rupture is a little later with wall friction, but the location of rupture is almost indistinguishable from the zero wall friction case. 


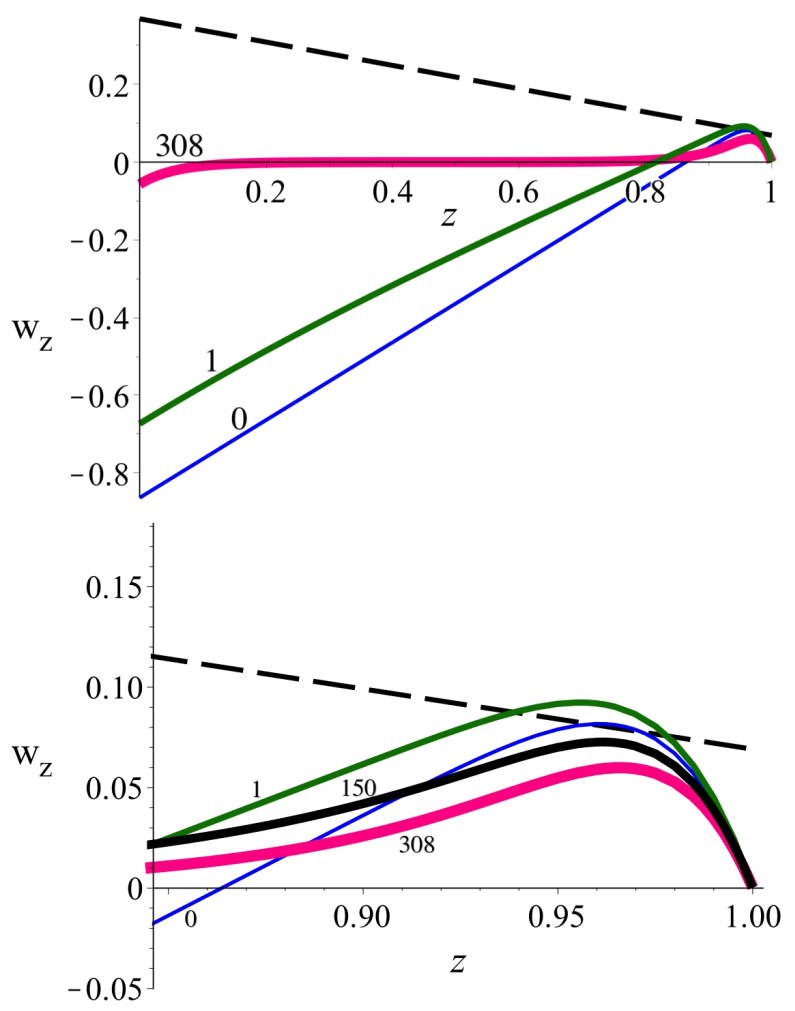

Fig. 7. Strain $w_{z}$ versus $z$, at dimensionless time $t=6 \times 10^{-4}$, compared with the tensile strength (dashed line). Wall friction is set to $\lambda_{f}=0,1,308$ (solid lines) in the first plot, with thicker lines for higher friction values. Other values are as listed in the tables. Strain increases with wall friction, away from $z=1$. The second plot shows a close-up of $\mathrm{z}$ near one, with an extra $\lambda_{f}=150$ value included - this value was not different enough to $\lambda_{f}=308$ to show in the first plot. $\lambda_{f}$ values are indicated near the associated curves. 


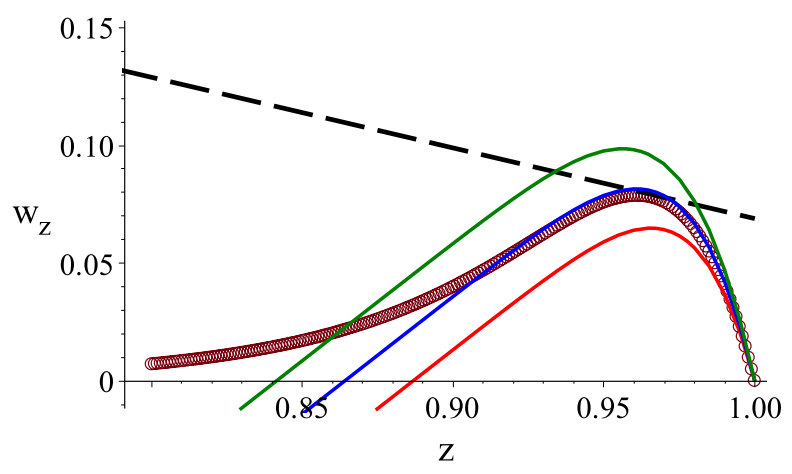

Fig. 8. A comparison of the dimensionless strain $w_{z}$ computed for wall friction $\lambda_{f}=308$ (circles) at dimensionless time $t=9 \times 10^{-4}$, with a wall friction set to zero (solid lines) at times $t=5,6,7 \times 10^{-4}$. The threshold for rupture is the dashed line. Rupture occurs at $t=6 \times 10^{-4}$ for zero wall friction, at almost the same location as it occurs for nonzero wall friction but at a different time.

\section{Varying Bead Size}

The effect of varying bead size on the size of the layers formed is explored here, using the zero wall friction case, since it is simpler and the depth of rupture appears to be almost the same as for nonzero wall friction. The main effect of changing bead size in our modelling, is to change the permeability of the bed - porosity is unaffected. There is also an effect on the elevation of the eruption threshold due to increased effective tensile strength with reduced bead size, which will become more significant for bead sizes less that 100 microns as van der Waals' forces become significant.

A formula approximating the dependence of layer size on bead size and overpressure can be obtained by considering an even simpler asymptotic expansion of the early time solution obtained by Laplace transforms in the previous section, by assuming $1-z$ is small.

If the approximate transform (42) is further expanded for small $1-z$, it becomes

$$
P \sim \operatorname{ap}_{a}\left(\frac{1-\sqrt{s}(1-z)+s(1-z)^{2} / 2}{s^{2}}\right), z \rightarrow 1,
$$


which inverts to give

$$
p \sim a p_{a}\left(t-2(1-z) \sqrt{\frac{t}{\pi}}+\frac{(1-z)^{2}}{2}\right), z \rightarrow 1, t \rightarrow 0,1-z \ll \sqrt{t} .
$$

Although this is a poor approximation for large $1-z$, and it tends to under-predict layer sizes, it provides a good estimate of overall trends in layer size, as will be seen in what follows.

Rupture of the bead bed occurs (ignoring wall friction) when

$$
p_{c}-p=1-z+S_{0 c}
$$

and when the slope of the pressure solution matches the slope of the overburden plus cohesion on the right-hand side of this equation, that is, there is just one root for the solution $1-z$. These considerations, using the early time simplification $p_{c} \sim a p_{a} t$, lead to the following two simultaneous equations for time of rupture $t_{r}$ and layer size $y=1-z$ :

$$
\begin{aligned}
\operatorname{ap}_{a}\left(2 y \sqrt{\frac{t_{r}}{\pi}}-\frac{y^{2}}{2}\right) & =S_{0 c}+y, \\
\operatorname{ap}_{a}\left(2 \sqrt{\frac{t_{r}}{\pi}}-y\right) & =\frac{d S_{0 c}}{d y}+1 .
\end{aligned}
$$

Noting that

$$
S_{0 c}=\left(\frac{1}{p_{0} \gamma \lambda}\right)\left(\frac{10^{-7}}{D_{p}^{2}}+\beta_{4} D_{p}+\kappa \sigma_{N c 0}+\beta_{5} y\right),
$$

where $\beta_{4}=\left(1-\phi_{0}\right) \rho_{s} g / 2 \approx 7350$, and $\beta_{5}=\kappa l \beta_{4} \approx 670$, the solution to these two equations is

$$
y=\sqrt{\frac{2}{a p_{a} p_{0} \gamma \lambda}\left(\frac{10^{-7}}{D_{p}^{2}}+\beta_{4} D_{p}+\kappa \sigma_{N C 0}\right)} .
$$


$p_{a}$ is nondimensional, so using

$$
a \tilde{p}_{a}=\frac{\lambda l \tilde{p}_{a}}{\mathrm{v}_{0} t_{c}}=\frac{l}{\mathrm{v}_{0} t_{c} \gamma}\left(\frac{p_{0}-p_{a}}{p_{0}}\right) .
$$

and

$$
\mathrm{v}_{0}=\frac{k_{0}\left(1-\phi_{0}\right) \rho_{s} g}{\eta \phi_{0}}
$$

and noting that $k_{0}=0.625 \times 10^{-3} D_{p}^{2}$, we see that, in terms of purely dimensional variables, slab thickness $Y=l y(\mathrm{~m})$ is given by

$$
Y^{2}=\beta_{6}\left(\frac{p_{0}}{p_{0}-p_{a}}\right)\left(10^{-7}+\beta_{4} D_{p}^{3}+\kappa \sigma_{N C 0} D_{p}^{2}\right)
$$

where

$$
\beta_{6}=\frac{1.25 \times 10^{-3} t_{c} \gamma}{\phi_{0} \eta}
$$

contains parameters independent of initial pressure and bead diameter.

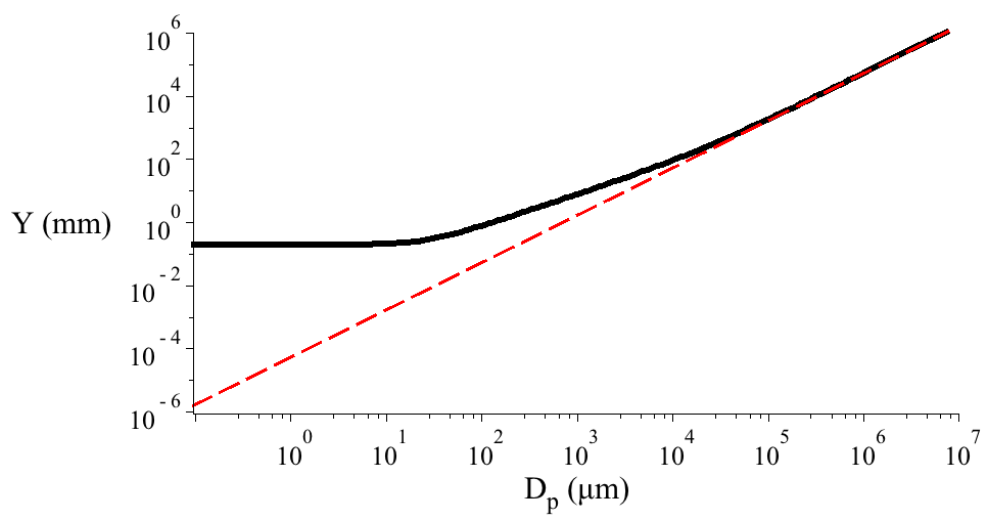

Fig. 9. Layer size $(\mathrm{mm})$ versus bead diameter $(\mu \mathrm{m})$ (solid line), according to the theoretical formula equation (52) with the choice $\sigma_{N C 0}=500$. Also shown in this plot (dashed line) is the large diameter approximation that layer size depends on diameter to the power 1.5 .

The resulting theoretical dependence of slab size on bead diameter is graphed as a log-log plot in Fig. (9, and can be seen to have three different regions, with 
constant slab size at very small diameters, a slope of one indicating a linear relationship between layer size and diameter at moderate diameters including those considered here, and a slope of 1.5 for large diameters indicating a power law of 1.5. The reasons for the shape of this plot are explored in more detail in the next paragraph.

There are three terms in the formula for the dependence of $Y^{2}$ on bead diameter. Which one is dominant, varies depending on the value of bead diameter. For diameters less than about $30 \mu$, the first term (due to $\sigma_{0}$, unconsolidated Lifschitzvan der Waals forces) is dominant, and slab thickness is predicted to be constant, independent of bead diameter.

For diameters between $30 \mu$ and $1 \mathrm{~cm}$, the last (quadratic) term is dominant, and slab thickness is predicted to be linear in bead diameter, as observed in Fig. (10). This term is due to any prior consolidation that might have taken place in charging the shock tube to initial pressure $p_{0}$. This has come about through the dependence of velocity on permeability which varies as the square of diameter. A simple explanation is that the similarity variable $y^{2} / t$ reaches a critical value $s_{c}$ at rupture, so that $y^{2} \propto s_{c} t$, and time scales as $1 / v \propto k \propto D_{p}^{2}$, giving $y \propto D_{p}$.

For diameters greater than $1 \mathrm{~cm}$, slab thickness is predicted to vary as diameter to the power 1.5, due to the middle (cubic) term in eqn 52 . It arises from the term $\sigma^{0}$, the geometric cohesion term.

The pressure normalization in this result (52) predicts that $Y$ is not very sensitive to overpressure. For example, if the shock tube is 3 bars over atmospheric, $\Delta p=(4-1) / 4=3 / 4$, while if the shock tube is 1 bar over atmospheric, $\Delta p=(2-1) / 2=1 / 2$. Taking square roots gives a relative change of layer thickness $Y$ from 3 bars to 1 bar as about $9 \%$. This insensitivity is arguably consistent with the observations of Anilkumar (1989, Table 3.4), where no dependence of layer size on initial over-pressure was observed over this range of over-pressures.

The dependence of $Y$ on bead diameter, according to this early-time, small $1-z$ approximate solution, is graphed in Fig. (10). As indicated by the approximate theoretical layer size (52), $Y$ increases monotonically with bead diameter, 
despite the increased cohesion at small diameters. This increase is exactly offset by the decrease in permeability as diameter decreases. This behaviour is in agreement with experimental results, although none of them explore the smaller bead diameter values.

Fig. (10) indicates that provided cohesion is taken to be large enough, a good agreement can be obtained between experimental values of layer size, and how they depend on bead size, and layer sizes predicted by our model.

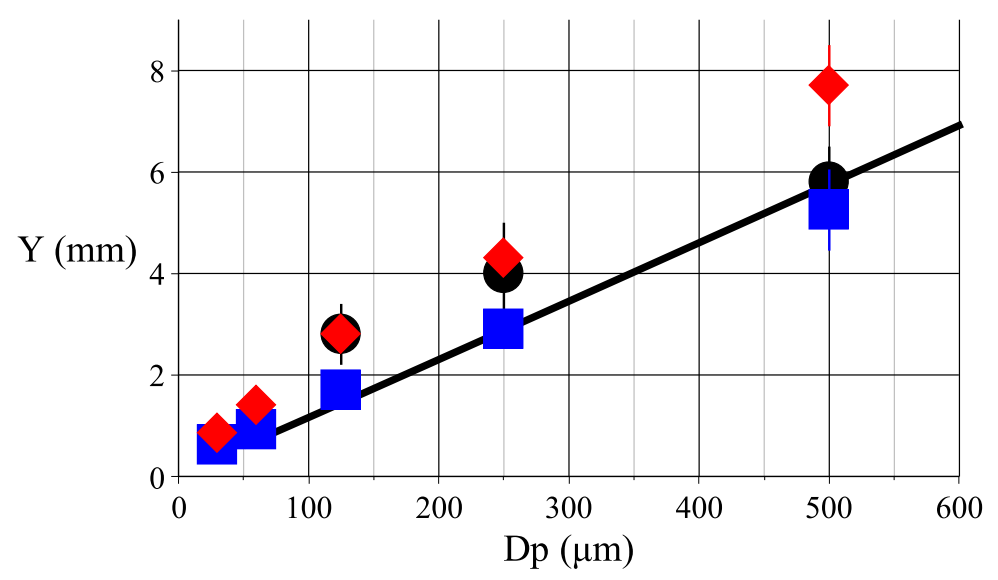

Fig. 10. Layer sizes versus bead diameter, showing the formula in the theoretical model equation (52) with the choice $\sigma_{N C 0}=1200$ (solid line), compared with Anilkumar's three experimental results (black disks), and more accurate numerical values obtained by using equations (48) and (49), using $\sigma_{N C 0}=1000$ (red diamonds) and $\sigma_{N C 0}=500$ (blue solid squares).

\section{Multiple Layers of Beads}

Some discussion is made here of the so-called stable layering of beads observed by Anilkumar (1989); Anilkumar et al (1993). If the bed is composed of three layers, all of the same diameter, with steel beads in the top one-thirds of the bed with density $7800 \mathrm{~kg} / \mathrm{m}^{3}$, glass in the middle one-third with density $2500 \mathrm{~kg} / \mathrm{m}^{3}$, and polystyrene in the lower one-third with density $1040 \mathrm{~kg} / \mathrm{m}^{3}$, then Anilkumar observes cracks forming initially, then closing up during subsequent 
upward movement. The variation in bead density with depth affects the cohesion $S_{0 c}$ by making it piecewise linear as illustrated in Fig. (11). The reduction process leading to a linear gas diffusion problem combined with the strain equation is not affected by the relatively small changes in density. The solution $p$ of the gas diffusion problem is the same as for a uniform bed of beads, since bead sizes are all the same. The gravity term 1 in the boundary-value problem (40) will change to a scaled density that is piecewise constant and of order one. Hence the shape of $w_{z}$ will remain the same as before, small for $z$ away from one and with a unique maximum near the place where $p$ is changing appreciably. The rupture criterion is slightly altered as reflected in Fig. (11), and a layer is still predicted to rupture away from the bed due to stress exceeding cohesion there.

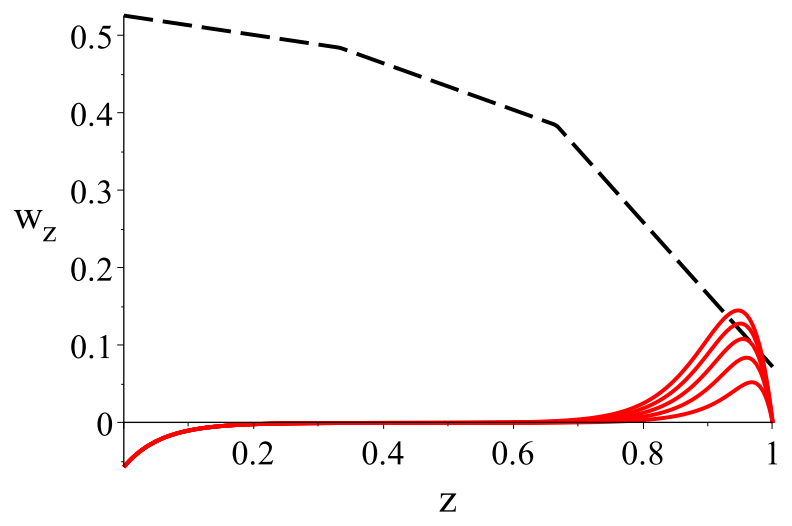

Fig. 11. Rupture condition for a three-layer bed with high density steel beads in the uppermost layer, glass in the middle layer, and lowest density polystyrene in the bottom layer. The dashed line shows the resulting cohesion $S_{0 c}$, to be exceeded by $w_{z}$ (solid lines, at evenly spaced dimensionless times from zero to $t=25 \times 10^{-4}$,) for bed rupture to occur. The strain $w_{z}$ is computed by solving the Greens function integration for $w_{z} z-\lambda_{f} w=\rho_{b}-p_{z}$, where $\rho_{b}$ is a normalised bead density, taking the value 0.4 for $z<1 / 3,1.0$ for $1 / 3 \leq z<2 / 3$, and 3.1 for $z>2 / 3$.

When three layers of different sized glass beads are placed in the bed, with the largest beads on top and the smallest beads at the bottom, similar behaviour is observed by Anilkumar (1989); Anilkumar et al (1993) — multiple cracks form 
initially, then close up during subsequent upward movement. Modelling this situation with our approach gives the same cohesion as illustrated in Fig. (5), since bed density and porosity are the same everywhere. Permeability is the most important parameter to change with particle size, so that the gas diffusion problem becomes one with varying permeability with depth. However, once again the general nature of the resulting solution will be a pressure $p$ that increases monotonically with height. The arguments presented in subsection (4.2) about the shape of $w_{z}$ apply, so that the general appearance of the rupture condition is similar to that illustrated in Fig. (5).

\section{Conclusions}

We have explained why layers form when a bed of dust is subjected to shocktube experiments, by developing, reducing, and solving a mathematical model for the conservation of mass and momentum for adiabatic compressible flow of gas through a porous medium of low cohesion, where the effect of gravity through overburden is taken to be important in the scalings used. The effects of wall friction and the nature of the cohesion of the bed have also been modelled.

The mathematical model reduces to two equations, a linear diffusion equation for pressure changes, which has been solved by elementary techniques, and a linear boundary-value problem for the steady-state solid displacement $w$, which can be solved once gas pressure is known. If wall friction is ignored, stress can be solved for analytically; otherwise a Green's function solution is provided that can be solved by numerical quadrature. General arguments for a unique local maximum in strain $w_{z}$ given the typical shape of a pressure diffusion problem have been made. This gives solid stress in the bed, and determines when and where cohesion is overcome.

Pressure drops at the surface of the bed when the expansion fan from the shock chamber reaches it. This drop diffuses into the bed, and lifts a layer off when the effective solid stress exceeds bed cohesion at some depth into the bed. In the absence of wall friction, this can be interpreted in terms of an increasing pressure 
difference that penetrates deeper into the bed as it grows, and eventually matches overburden plus cohesion at some depth, whereupon a layer of dust ruptures and lifts off. This process is then set to repeat again and again, rupturing the dust bed in regular layers.

The least well-determined parameter in the model is the cohesion, and this parameter was adjusted within a reasonable range, to obtain a good match to experimental results.

Once a single layer lifts off, previous work (Fowler et al., 2009; McGuinness et al., 2012) shows that to a good approximation the pressure at the surface of the remaining stationary bed might be anticipated to follow $p_{c}$, so that the analysis giving the rupture of one layer applies again and again, giving multiple layers in succession, all of similar size since the only parameter that is changing is the length $l$ of the remaining bed.

The dependence of layer size on bead diameter is explored using a small time small $1-z$ approximation that gives a good match to experimental values, and to numerical experiments using a more accurate pressure solution. In contrast to Anilkumar's speculation that layer size varies as the square root of bead diameter (Anilkumar, 1989), we find a theoretical basis for layer size to be proportional to bead diameter, in the range of diameters considered, which is a good fit to Anilkumar's results. Further experimentation with larger and smaller bead diameters and different over-pressures would be useful to verify our layer size predictions.

Our modelling results are consistent with reported observations of layered beds, with heavier beads above or below lighter beads, or with larger beads above or below smaller beads, in that bed rupture is always predicted to occur for large enough values of $p_{0}$. Subsequent repacking, where in some (stable) bed configurations the layers move closer and close up the gaps between, is then due to the kinematics of the relative layer speeds, although we do not attempt to explore this here.

Cohesion is also critically dependent on moisture content and static electricity charges. The theory presented here provides a framework for theoretical inves- 
tigations into the dependence of layer formation on moisture content, provided that theoretical development of the effect of moisture on cohesion is made, which would be very interesting to support and verify with further experimental work.

\section{Acknowledgments}

We are grateful to Professor Andrew Fowler (Math. Inst., University of Oxford and MACSI, University of Limerick) for fruitful discussions that helped us to progress this work; and to Professor Colin Wilson at Victoria University of Wellington for the comments that started our interest in erupting dusts; and to Professor Amrutur V. Anilkumar, Department of Mechanical Engineering, Vanderbilt University, for permission to use his erupting dust figures.

Abramowitz, M., \& Stegun, I., 1972. Handbook of Mathematical Functions. Dover, NY.

Alidibirov, M., 1994. A model for viscous magma fragmentation during volcanic blasts. Bulletin of Volcanology 56, 459-465.

Anilkumar, A.V., 1989. Experimental studies of high-speed dense dusty gases. Ph.D. Thesis, Aeronautics, California Institute of Technology, Pasadena, CA, USA.

Anilkumar, A.V., Sparks, R.S.J., Sturtevant, B., 1993. Geological implications and applications of high-velocity two-phase flow experiments, J. Volcanol. Geoth. Res., 56, 145-160.

Biot, M.A., 1956. Theory of propagation of elastic waves in a fluid-saturated porous solid. I. Low-frequency range. J. Acoust. Soc. Amer. 28, 168-178.

Biot, M.A., 1962. Mechanics of deformation and acoustic propagation in porous media. J. Appl. Phys. 33, 1,482-1,498. 
Cagnoli, B., Barmin, A., Melnik, O., Sparks, R.S.J., 2002. Depressurization of fine powders in a shock tube and dynamics of fragmented magma in volcanic conduits Earth \& Planetary Sci. Lett. 204, 101-113.

Cashman, K.V., Sturtevant, B., Papale, P., Navon, O., 2000. Magmatic fragmentation. In: Encyclopedia of Volcanoes, Ed. Sigurdsson, H., Academic Press, SanDiego, 421-430.

Coelho, D., Thovert, J.-F., and Adler, P.M., 1997. Geometrical and transport properties of random packings of spheres and aspherical particles Phys. Rev. E, v. 55, No. 2, 1959-1978.

Costa, A., 2006. Permeability-porosity relationship: A re-examination of the Kozeny-Carman equation based on a fractal pore-space geometry assumption. Geophys. Res. Lett. 33: L02318. doi:10.1029/2005GL025134

Crank, J., 1975. The Mathematics of Diffusion, 2nd Edn, Clarendon Press, Oxford.

Dartevell, S., and Valentine, G.A. (2007), Transient multiphase processes during the explosive eruption of basalt through a geothermal borehole (Nmafjall, Iceland, 1977) and implications for natural volcanic flows. Earth and Planetary Sci. Lett. 262, 363-384.

Emery, E., Oliver, J., Pugsley, T., Sharma, J., \& Zhou, J., 2009. Flowability of moist pharmaceutical powders. Powder Tech. 189, 409-415.

Ergun, S.,1952. Chem. Eng. Prog., 48, 89p.

Fowler, A.C., Scheu, B., Lee, W.T., McGuinness, M.J., 2009. A theoretical model of the explosive fragmentation of vesicular magma. Proc. Roy. Soc. Lond., A., March 8, 2010, 466:731-752; published online before print November 3, 2009. doi:10.1098/rspa.2009.0382

Geldart, D., 1973. Powder Technol., 7, 285. 
Gilbert, J.S., Sparks, R.S.T. (eds), 1998. The physics of explosive volcanic eruptions. Geological Soc. Special Publications No. 145, London, UK: Geological Society.

Heiken G., Wohletz, K., 1991. Fragmentation processes in explosive volcanic eruptions. In: Sedimentation in volcanic settings, Society for Sedimentary Geology, Special Publications No. 45, 19-26.

Ichihara, M., Rittel, D., Sturtevant, B., 2002. Fragmentation of a porous viscoelastic material: implications to magma fragmentation. J. Geophys. Res. 107 (B10), 2229. doi:10.1029/2001JB000591

Jaraiz, E., Kimura, S., Levenspiel, O., 1992. Vibrating beds of fine particles: estimation of interparticle forces from expansion and pressure drop experiments. Powder Tech. 72, 23-30.

Kameda, M., Kuribara, H., Ichihara, M., 2008. Dominant time scale for brittle fragmentation of vesicular magma by decompression. Geophys. Res. Lett. 35, L14302. doi:10.1029/2008GL034530

Mader, H.M., Zhang, Y., Phillips, J.C., Sparks, R.S.J., Sturtevant, B., Stolper, E., 1994. Experimental simulations of explosive degassing of magma. Nature 372, 85-88.

Mangan, M.T., Cashman, K.V., 1996. The structure of basaltic scoria and reticulite and inferences for vesiculation, foam formation, and fragmentation in lava fountains. J. Volcanol. Geoth. Res., 73, 1-18.

Martel, C., Dingwell, D.B., Spieler, O., Pichavant, M., Wilke, M., 2000. Fragmentation of foamed silicic melts: an experimental study. Earth and Planetary Science Letters 178, 47-58.

McBirney, A.R., Murase, T., 1970. Factors governing the formation of pyroclastic rocks. Bull. Volc. 34, 372-384. 
McGuinness, M.J., Scheu, B., Fowler, A.C., 2012. Explosive fragmentation criteria and velocities for vesicular magma, J. Volc. and Geothermal Res., 237238, 81-96. http://dx.doi.org/10.1016/j.jvolgeores.2012.05.019 or http://www.sciencedirect.com/science/article/pii/S0377027312001576

Mikami, T., Kamiya, H., \& Horio, M., 1998. Numerical simulation of cohesive powder behaviour in a fluidised bed. Chem. Eng. Science 53, No. 10, 19271940.

Molerus, O., 2002. The role of science in particle technology. Powder Tech. 122, $156-167$.

Molerus, O., 1993. Principles of Flow in Disperse Systems. Chapman \& Hall, London, 1993.

Namiki, A., Manga, M., 2005. Response of a bubble bearing viscoelastic fluid to rapid decompression: implications for explosive volcanic eruptions. Earth and Planetary Science Letters 236, 269-284.

Orband, J.L.R. \& Geldart, D., 1997. Direct measurement of powder cohesion using a torsional device. Powder Tech. 25-33.

Phillips, J.C., Lane, S.J., Lejeune, A.M., Hilton, M., 1995. Gum rosin-acetone system as an analogue to the degassing behaviour of hydrated magmas. Bulletin of Volcanology 57, 263-268.

Scheu, B., Spieler, O., Dingwell, D.B., 2006. Dynamics of explosive volcanism at Unzen volcano: an experimental contribution. Bulletin of Volcanology 69 (2), $175-187$.

Scheu, B., Ichihara, M., Spieler, O., Dingwell, D.B., 2008. A closer look at magmatic fragmentation. Geophys. Res. Abst. 10, EGU2008-A-04786.

Sparks, R.S.J., 1978. The dynamics of bubble formation and growth in magmas: a review and analysis. J. Volc. Geoth. Res. 3, 1-37. 
Spieler, O., Kennedy, B., Kueppers, U., Dingwell, D.B., Scheu, B., Taddeucci, J., 2004b. The fragmentation threshold of pyroclastic rocks. Earth and Planetary Science Letters 226, 139-148.

Tanneur, V., Joussot-Dubien, C., Fournel, B., Sarrade, S., Freiss, B., Marciacq, F., \& Rios, G.M., 2008. Group A particle fluidization in supercritical carbon dioxide: Effect of operating conditions on fluidization efficiency. Powder Tech. 187, 190-194.

Valverde, J.M., Ramos, A., Castellanos, A., \& Watson, P.K., 1998. The tensile strength of cohesive powders and its relationship to consolidation, free volume and cohesivity. Powder Tech. 97, 237-245.

Verhoogen, J., 1951. Mechanics of ash formation. Am. J. Sci. 249, 729-739.

Weber, M.W., 2004. Simulation of cohesive particle flows in granular and gassolid systems, a thesis submitted for the degree of Doctor of Philosophy, Dept. of Chemical and Biological Eng., University of Colorado.

Weir, G.J., 1999. The intrinsic cohesion of granular materials. Powder Tech. 104, 29-36.

Xu, C., \& Zhu, J., 2006. Parametric study of fine particle fluidization under mechanical vibration. Powder Tech. 161, 135-144. 